\title{
Impulse Radar Imaging for Dispersive Concrete Using Inverse Adaptive Filtering Techniques
}

\author{
Joe Arellano
}

Jose M. Hernandez

James Brase

May, 1993

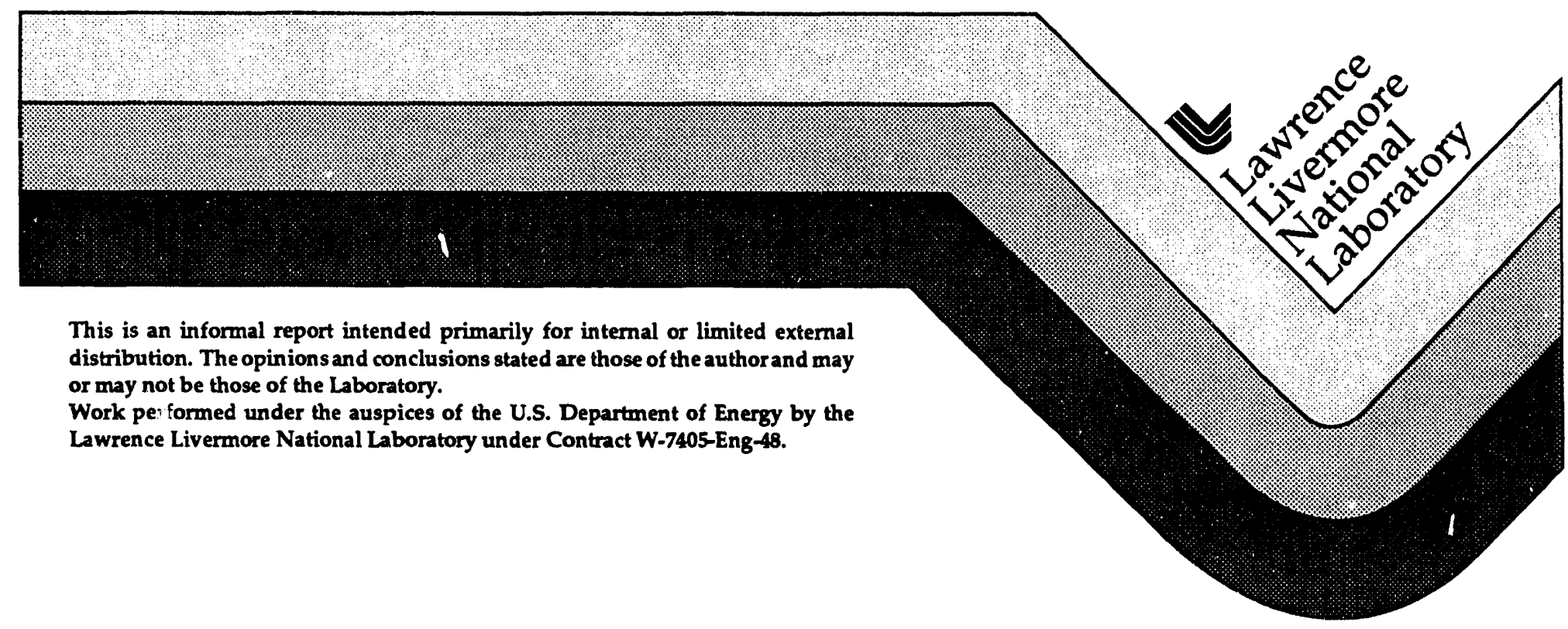




\section{DISCLAIMER}

This document was prepared as an account of work sponsored by an agency of the United States Government. Neither the United States Government nor the University of California nor any of their employees, makes any warranty, express or implied, or assumes any legal liability or responsibility for the accuracy, completeness, or usefulness of any information, apparatus, product, or process disclosed, or represents that its use would not infringe privately owned rights. Reference herein to any specific commercial products, process, or service by trade name, trademark, manufacturer, or otherwise, does not necessarily constitute or imply its endorsement, recommendation, or favoring by the United States Government or the University of California. The views and opinions of authors expressed herein do not necessarily state or reflect those of the United States Government or the University of California, and shall not be used for advertising or product endorsement purposes.

This report has been reproduced directly from the best available copy.

Available to DOE and DOE contractors from the Office of Scientific and Technical Information P.O. Box 62, Oak Ridge, TN 37831

Prices available from (615) 576-8401, FTS 626-8401

Available to the public from the National Technical Information Service

U.S. Department of Commerce 5285 Port Royal Rd. Springfield, VA 22161 


\section{Contents}

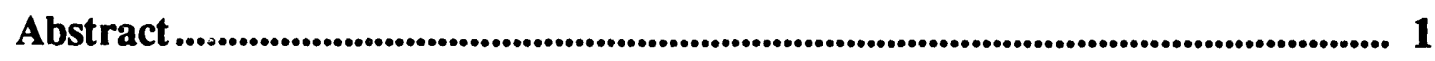

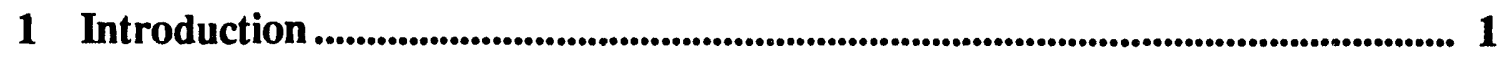

2 Multiple-Target Non-Dispersive Concrete .................................................................... 3

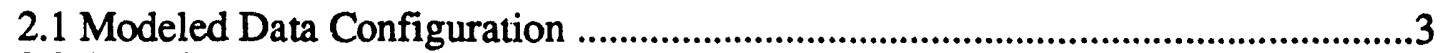

2.2 Adaptive Filter Configuration ..........................................................................

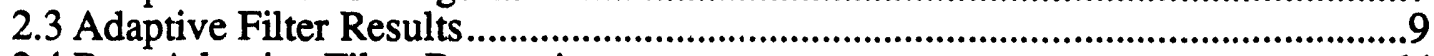

2.4 Post-Adaptive Filter Processing ................................................................................... 11

3 Variable Size Single Target in Dispersive Concrete .............................................. 17

3.1 Modeled Data Configuration ........................................................................... 17

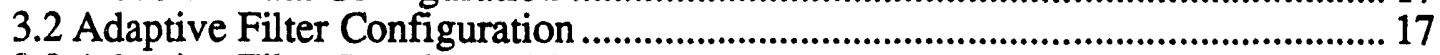

3.3 Adaptive Filter Results and Post-Adaptive Filter Processing ................................ 18

4 Variable Depth Single Target in Dispersive Concrete............................................... 27

4.1 Modeled Data Configuration ...................................................................................... 27

4.2 Adaptive Filter Configuration and Resultant Images ………………………….... 27

5 Variable Pulse-Width Transmitted Signal with Single Target in Dispersive Concrete ........................................................................................................................ 33

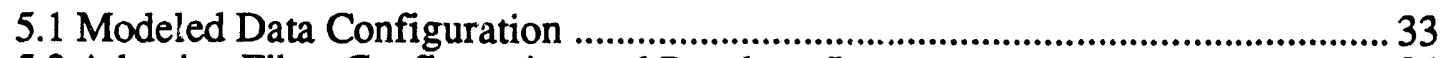

5.2 Adaptive Filter Configuration and Resultant Images ........................................... 34

6 Time Domain Synthetic Aperture Focusing Technique ............................................. 43

7 Resolution, Sampling, and Signal-to-Artifact Ratio ...................................................45

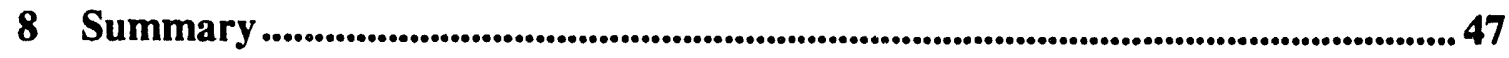

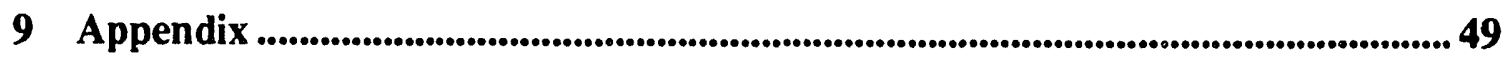

9.1 Program Listing of Adaptive Filter Algorithm ..................................................49

9.2 Program Listing for Time-Domain Synthetic Aperture Focusing ……………..... 53

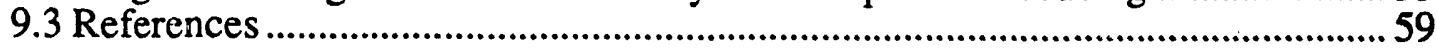




\section{Figures}

\section{Multiple Target Data}

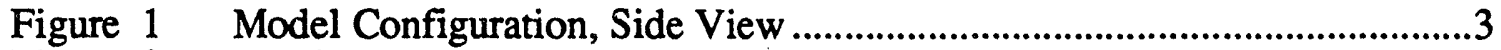

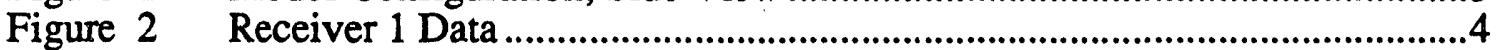

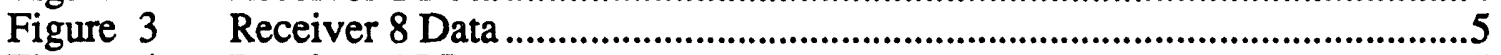

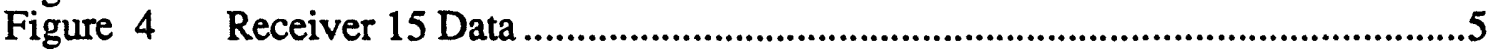

Figure 5 Receiver 1 Data Without Coupling Pulse .............................................6

Figure 6 Receiver 8 Data Without Coupling Pulse ................................................6

Figure 7 Receiver 15 Data Without Coupling Pulse .........................................6

Figure 8 Delayed Adaptive Inverse Model ......................................................7

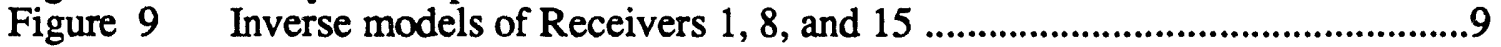

Figure 10 Convolution of $p$ Filter and Weights for Receivers 1, 8, and $15 \ldots \ldots \ldots \ldots . . .10$

Figure 11 MSE for the Adaptive Filter for Receivers 1, 8, and $15 \ldots \ldots \ldots \ldots \ldots \ldots \ldots \ldots . . . . . . . .10$

Figure 12 Filtered Three Target Data for Receivers 1, 8, and 15.......................... 11

Figure 13 Frequency Transforms for Post-Adaptive Filter Signal Processing........... 12

Figure 14 Focused Data from Adaptive, Blackman and Notch Filters ....................... 13

Figure 15 Modified Blackman Window .............................................................. 14

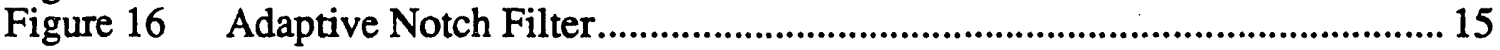

\section{Variable Size Single Target Data}

Figure 17 Receiver 1 Single Void Data With And Without Coupling Pulse ............. 18

Figure 18 Standard Weights for 35-mm Void from Receiver 1 ................................. 19

Figure 19 Convolution Results of $p$ Filter and Weights ........................................... 19

Figure 20 MSE for the Adaptive Filter............................................................ 19

Figure 21 Image of 5-mm Void Before and After Adaptive Filter Weights ............... 21

Figure 22 Image of 35-mm Void Before and After Adaptive Filter Weights ............. 22

Figure 23 Image of 55-mm Void Before and After Adaptive Filter Weights ............ 23

Figure 24 Image of 75-mm Void Before and After Adaptive Filter Weights ............. 24

Figure 25 Comparison of 3 75-mm Void Size Images ........................................... 25

\section{Variable Depth Single Target Data}

Figure 26 Receiver 1 Single Void Data with and Without Coupling Pulse ............... 28

Figure 27 Standard Weights for 80-mm Depth Void from Receiver 1 ....................... 28

Figure 28 Convolution Results of $p$ Filter and Weights ......................................... 28

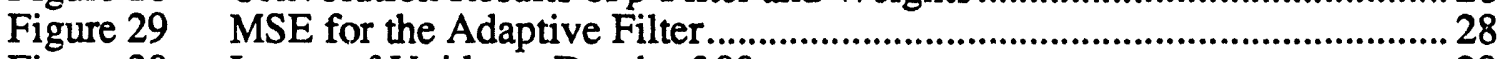

Figure 30 Inage of Void at a Depth of $80 \mathrm{~mm}$...................................................... 29

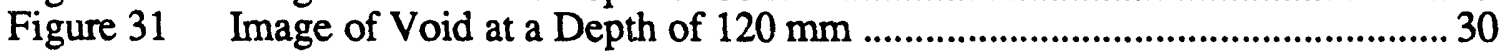

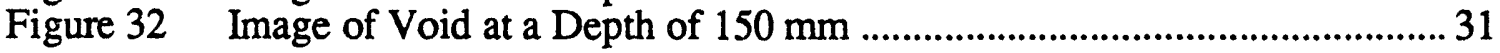




\section{Variable Pulse-Width Single Target Data}

Figure 33 Block Diagram of Concrete for Obtaining Impulse Response .................. 33

Figure 34 Rebar Image with 100-ps Transmitted Pulse-Width ................................... 35

Figure 35 Rebar Image with 500-ps Transmitted Pulse-Width .................................. 36

Figure 36 Rebar Image with 1000-ps Transmitted Pulse-Width ..................................... 37

Figure 37 Receiver 1 1000-ps Transmitted Pulse-Width, Single Rebar Data ............. 38

Figure 38 1000-ps Transmitted Pulse ........................................................................ 39

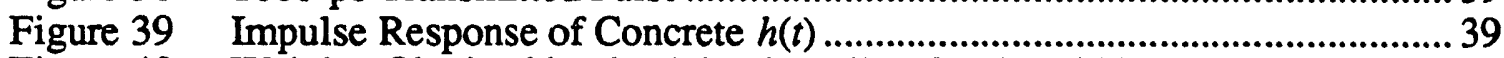

Figure 40 Weights Obtained by the Adaptive Filter for the 1000-ps Pulse .................. 39

Figure 41 Convolution Results of $p$ Filter and Weights ........................................ 39

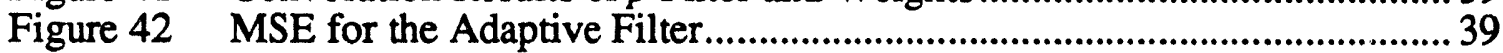

Figure 43 Rebar Image with 1000-ps Pulse Using Impulse Response ....................... 40

Figure 44 Comparison of 1000-ps Transmitted Pulse Images .................................. 41

\section{Time Domain Synthetic Aperture Focusing Technique}

Figure 45 Bistatic Focusing Technique 43

Figure 46 Bistatic Focusing Technique with Multiple Receivers

\section{Resolution and Signal-to-Artifact Ratio}

Figure 47 Bistatic Model of Transmitter and Receivers 


\begin{abstract}
This publication addresses applications of a delayed inverse model adaptive filter for modeled data obtained from short-pulse radar reflectometry. To determine the integrity of concrete, a digital adaptive filter was used, which allows compensation of dispersion and clutter generated by the concrete. A standard set of weights produced by an adaptive filter are used on modeled data to obtain the inverse-impulse response of the concrete. The data for this report include:

- Multiple target, nondispersive data.

- Single-target, variable-size dispersive data.

- Single-target, variable-depth dispersive data.

- Single-target, variable transmitted-pulse-width dispersive data.

Results of this simulation indicate that data generated by the weights of the adaptive filter, coupled with a two-dimensional, synthetic-aperture focusing technique, successfully generate two-dimensional images of targets within the concrete from modeled data.
\end{abstract}

\title{
Introduction
}

Lawrence Livermore National Laboratory (LLNL) is currently sponsoring a project to study short-pulse, wide-band reflectometry from concrete in roads and bridges. The purpose of this project is to develop nondestructive techniques to determine damage to the concrete by means of detecting voids and reinforcing bars (rebars). Reflected signals from single and multiple-targets can be analyzed to determine the location and type of scatterer. Unfortunately, reflected signals obtained by the receivers contain clutter from reflections of aggregate within the concrete as well as target information. The majority of the modeled data in this report considers dispersion because it considerably affected the received signals.

Although several methods have been developed for determining target location within concrete, ${ }^{[1,2]}$ the methods explained in this paper will obtain the inverse-impulse response of the medium. By simply convolving the inverse-impulse response with the model data dispersion, clutter and other affects can be taken into consideration. Additionally, other signal analysis not considered in this report can be performed on the inverse-impulse response waveforms.

Three important assumptions were made in generating and analyzing the modeled data. First, the transmitters and receivers were placed idealistically within the concrete pad. This placement eliminated any front surface reflections and effectively simplified the analysis of the collected data. Second, the signal coupled between transmitter and receivers was removed. That is, each data set was supplied with data which contained notarget reflections, thus the coupling between the transmitter and receivers was removed by subtracting the no-target data from the target data. Finally, the transmitted pulse was considered to be a wide-band pulse, i.e. having the approximate shape of an impulse. These 
three assumptions have simplified the analyses of the data and will require eventual consideration. However, these assumptions are also reasonable and should not present any insurmountable problems.

Several different sets of data are evaluated in this report. The data set in section 2 considered multiple-target non-dispersive concrete. Weights were generated from a single target and then applied in a general sense to multiple target data. Other signal processing tools are also discussed. The data containing dispersive effects are discussed in sections 3,4 , and 5. These respectively include:

- Single-target, variable-size data.

- Single-target, variable-depth data.

- Single-target, variable transmittedpulse-width data.

Because the transmitted pulse is not considered a wide-band signal in the third bullet data set, alternate methods for obtaining the inverse-impulse response from the adaptive filter are also discussed in section 5.

Section 6 examines the time-domain synthetic-aperture focusing technique (SAFT). This technique is used to generate the twodimensional images in this report obtain by the fifteen receivers considered in the modeled data. After accounting for the location of the receivers, the procedure requires that filtered data be swept across a two-dimensional plane. Signals from the receivers additively accumulate in the correct location to produce an image.

Section 7 briefly describes the resolution, sampling and signal-to-artifact points and the calculations presented in this section were determined from first-order approximations. 


\section{Multiple-target Nondispersive Concrete}

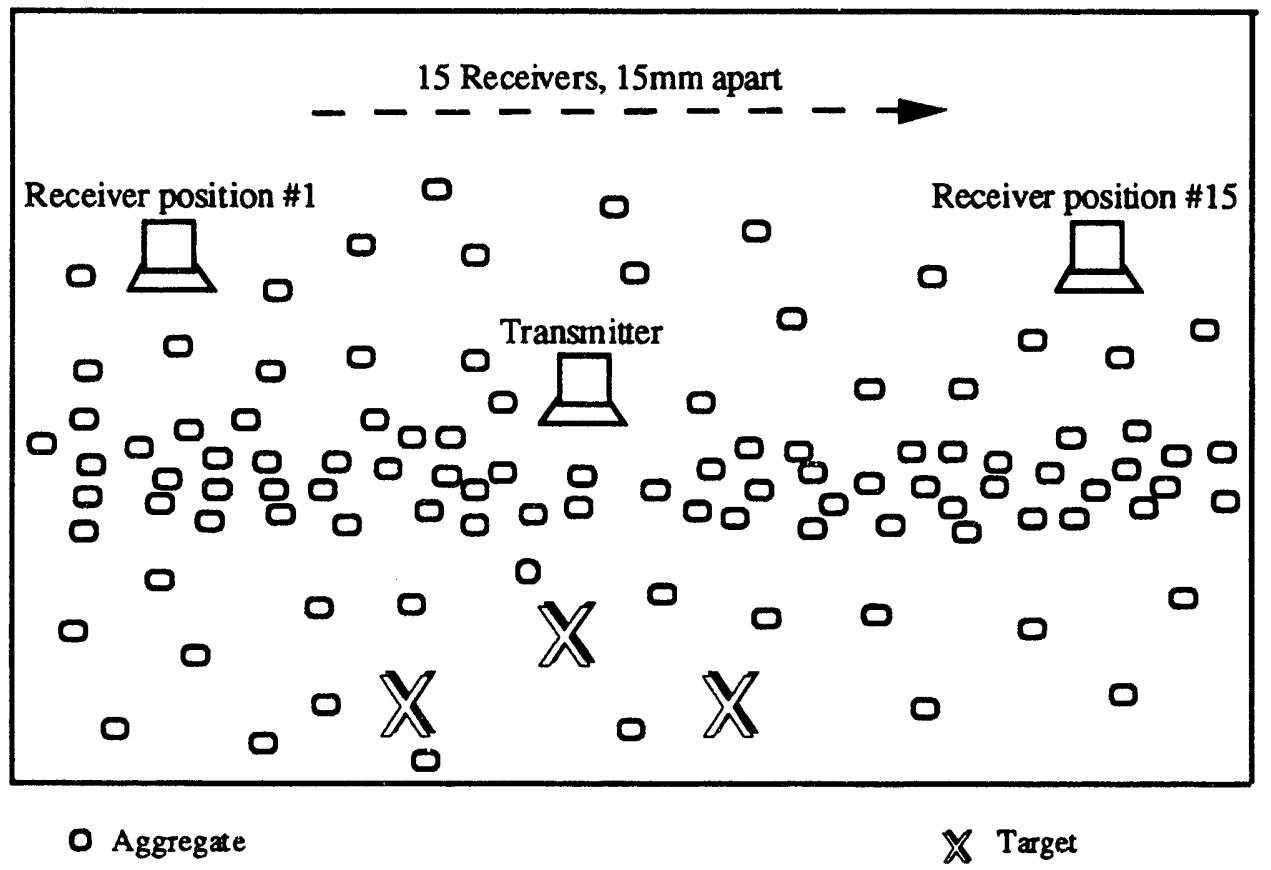

Figure 1. Model configuration, side view.

\subsection{Modeled Data Configuration}

Figure 1 shows the configuration of the model used to generate data for this experiment. The concrete was pulsed with a wide-band electromagnetic signal while the transmitter was located approximately in the center of the concrete pad. The receivers, separated by $15 \mathrm{~mm}$, were also positioned above the targets. Although the transmitter and receivers were held stationary for this data, eventual system implementation will require the transmitter and receivers to be located on a movable platform as an image is generated from the targets in the concrete. In effect, a synthetic-aperture image is generated. [3]

Model parameters to generate data for this section include modeling concrete with approximately 1800 rocks scattered throughout the material, with most of the rocks confined to an area near the surface. The transmitted pulse was modeled as a Gaussian-shaped pulse with a full-width, half-maximum amplitude of 100 ps. In this model, the relative dielectric constant was kept constant at 6 for the concrete and 4.3 for the rocks. Data collected from the receivers had a data sampling rate of $20 \mathrm{ps.}$

Three data subsets were generated from the receivers in Figure 1. The first subset had no targets in the concrete, the second had only a single void, and the third had three targets. 
Figures $2 a-c, 3 a-c$, and $4 a-c$ show waveforms generated for receiver position numbers 1,8 , and 15 , respectively. These waveforms were chosen as representative signals to thoroughly demonstrate the effectiveness of the adaptive filter.

In Figures 2a, 3a, and 4a, the initial portion of the received waveform contains a large pulse created by direct signal coupling between the transmitter and the receivers. The coupling pulse variations are caused by the difference in distance the transmitted signal must travel between receivers. Note that the similarities between Figures $2 a$ and $4 a$ are caused by the symmetry of the system, i.e., the transmitter is located in the center, and receiver positions 1 and 15 are in the outermost locations of the system.

The oscillations in Figures 2a and 4a, located at sample number 100 , are caused by the initial reflections of the aggregate. The same reflections are at sample number 70 for Figure 3a. The disturbances in Figures 2a, 3a, and $4 a$ at sample 250 are created by the back edge boundary of the model. For Figure $2 b$, perturbations in the signal for a single target are located at sample 180, for Figure $3 \mathrm{~b}$ at 200, and for Figure $4 \mathrm{~b}$ at 225 . Three-target information is centered at sample number 200 for Figure 2c, sample number 180 for Figure 3c, and sample number 200 for Figure 4c. From these figures it is apparent how the ringing of the signal caused by the reflection of a single target is coupled to the reflections generated by the other targets.

To remove the direct coupling pulse between the transmitter and the receivers, the no-target data was subtracted from the multipletarget and single-target data, using the appropriated position data. These signals are shown in Figures 5, 6, and 7 for positions 1,8, and 15 , respectively. Because the no-target data had the same pattern of aggregate as the target data, the aggregate was also removed. The removal of the aggregate was considered inconsequential because the major reflection caused by the aggregate occurs much earlier then the reflection caused by the target. Consequently, the results of the adaptive filter should not be affected if the filter is allowed to converge only on the portion of the signal containing the target reflection.

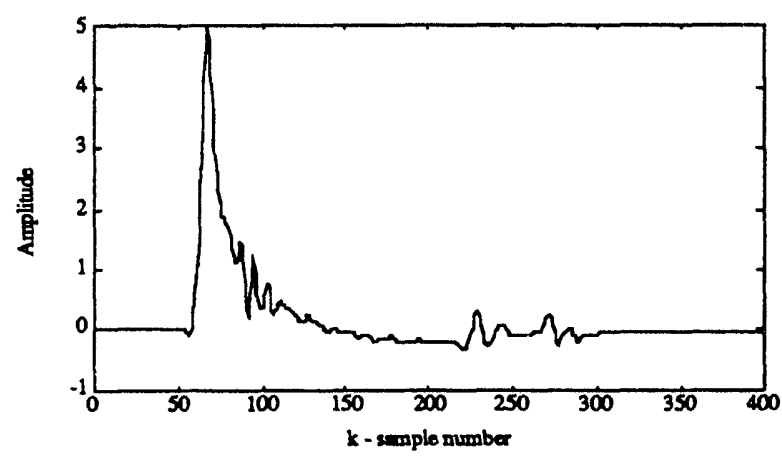

(a)

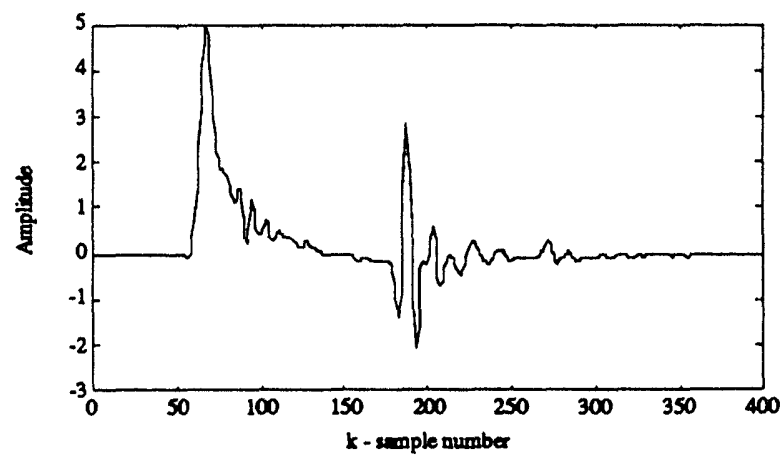

(b)

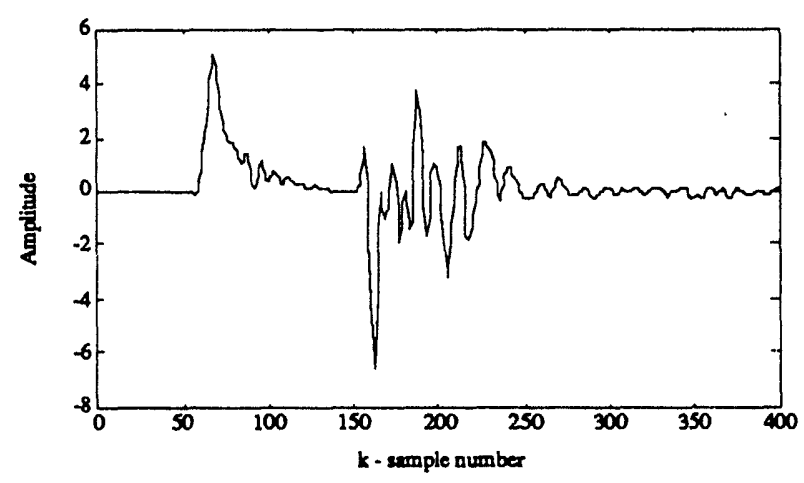

(c)

Figure 2. Receiver 1 signal for (a) no target data, (b) single-target data, and (c) threetarget data. 


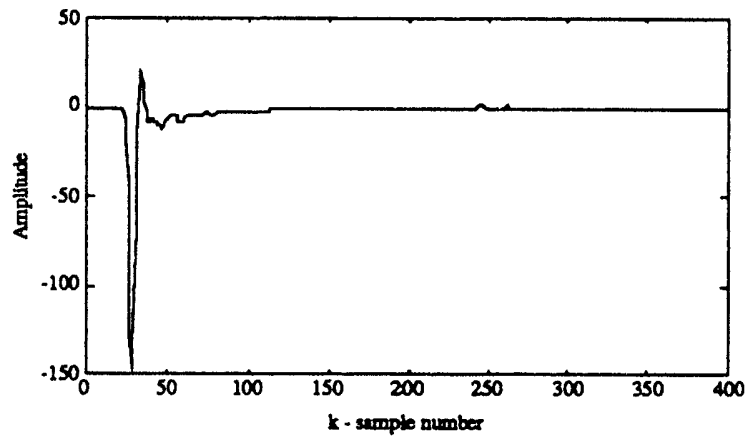

(a)

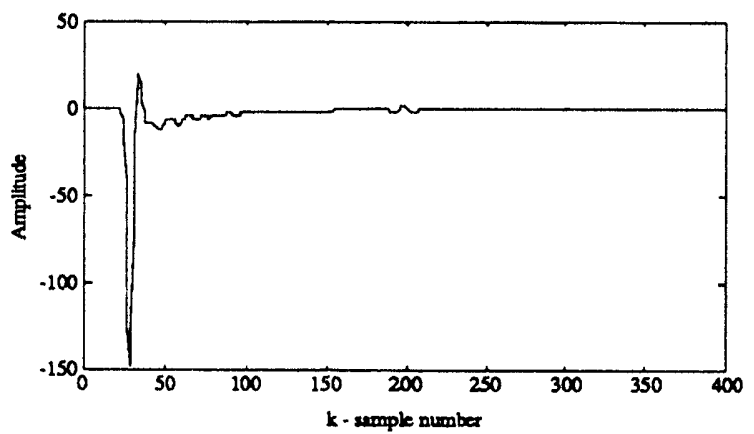

(b)

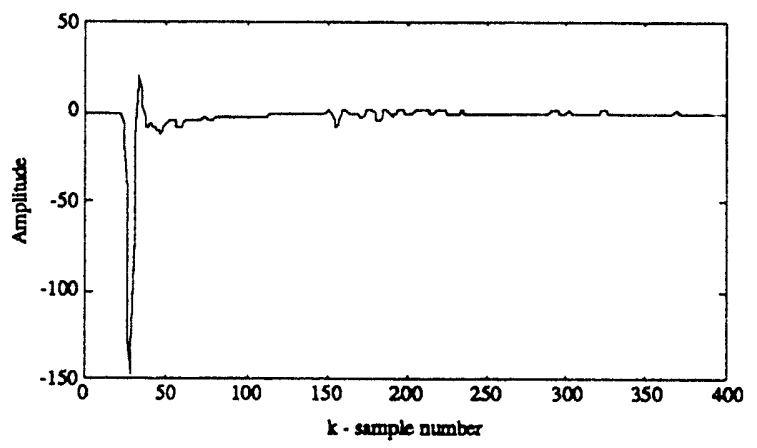

(c)

Figure 3. Receiver 8 signal for (a) no-target data, (b) single-target data, and (c) threetarget data.

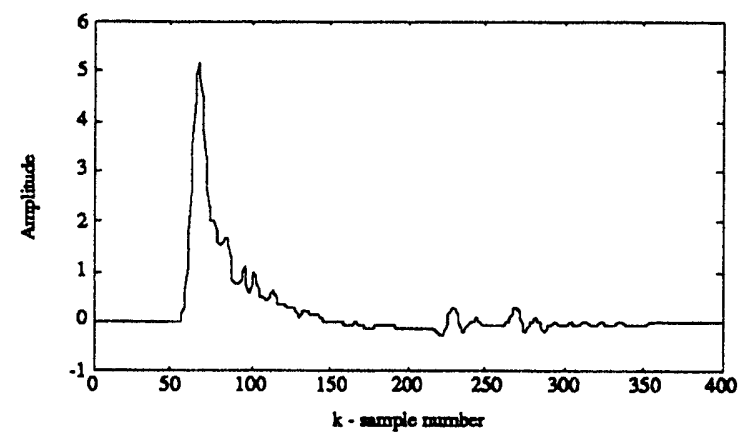

(a)

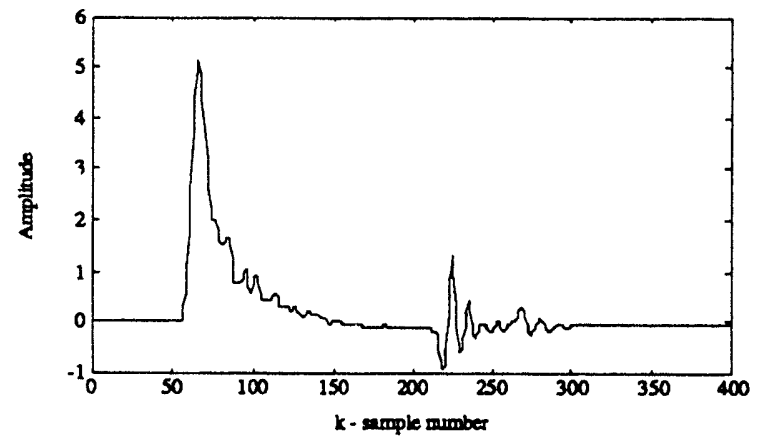

(b)

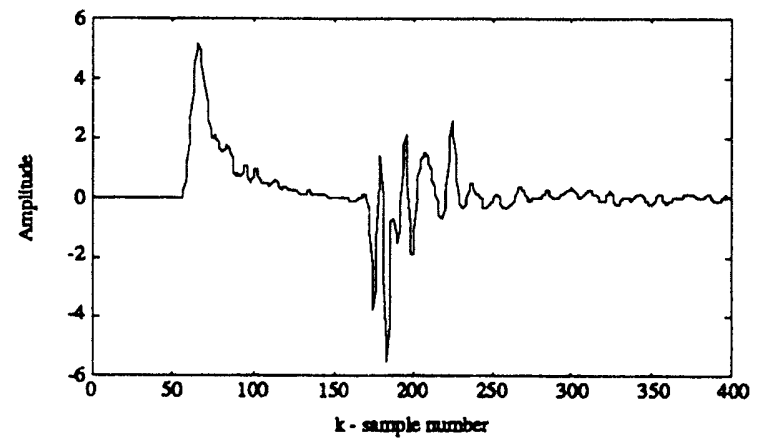

(c)

Figure 4. Receiver 15 signal for (a) no-target data, (b) single-target data, and (c) threetarget data. 


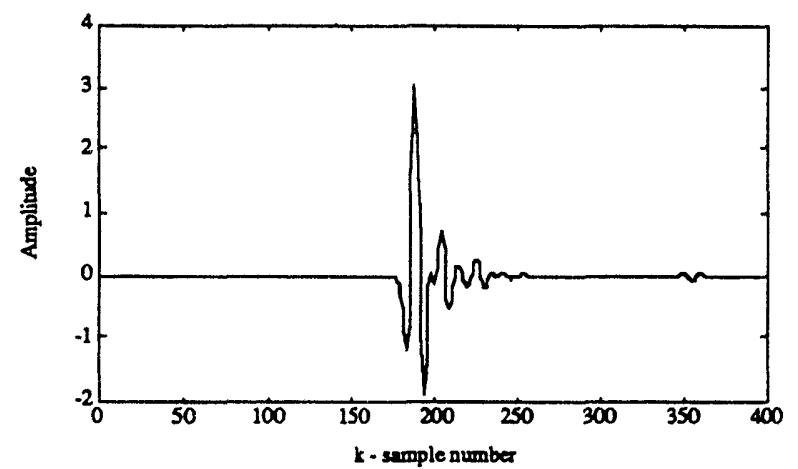

(a)

Figure 5. Receiver 1 without coupling pause for (a) single-target data, and (b) threetarget data.

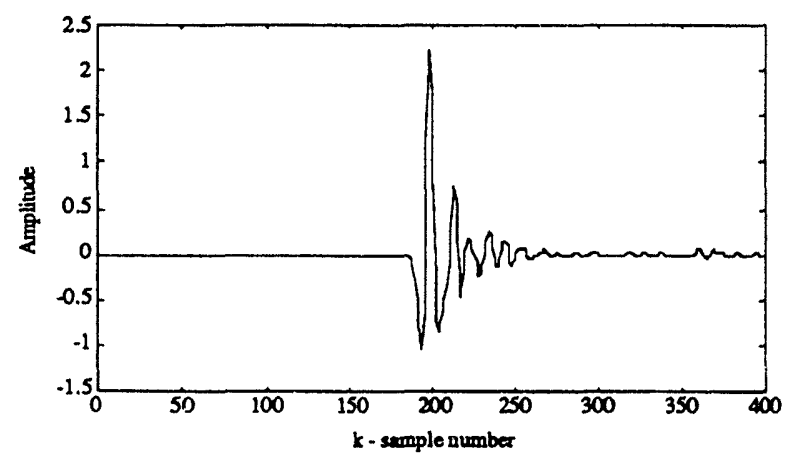

(a)

Figure 6. Receiver 8 without coupling pulse for (a) single-target data, (b) three-target data.

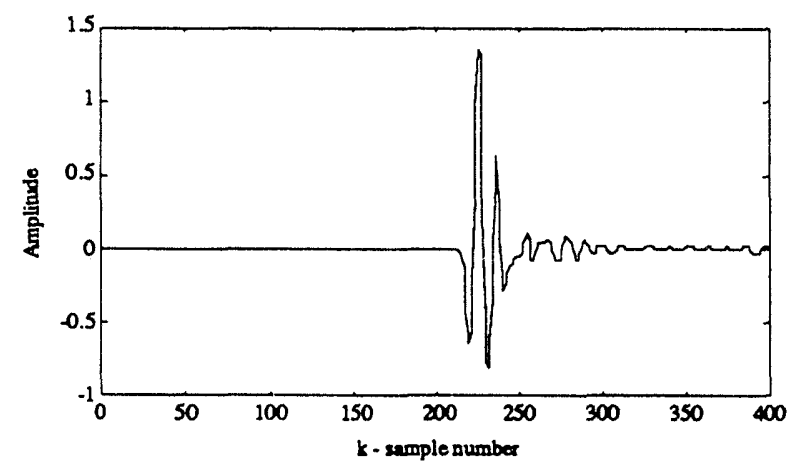

(a)

Figure 7. Receiver 15 without coupling pulse for single-target Single-target data without coupling pulse.

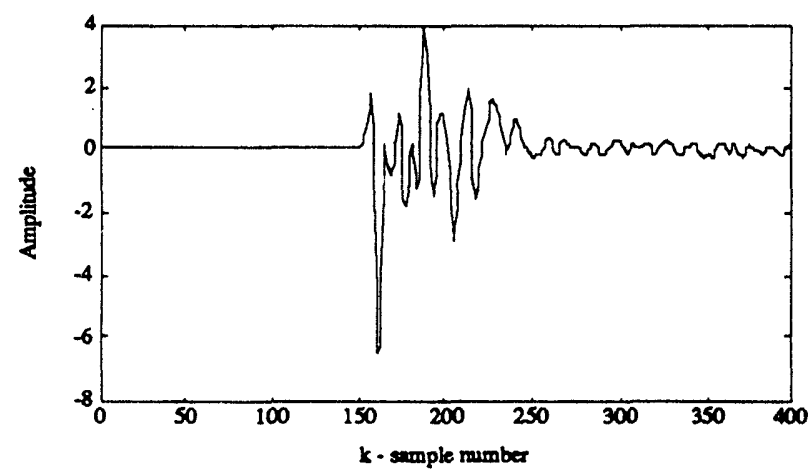

(b)

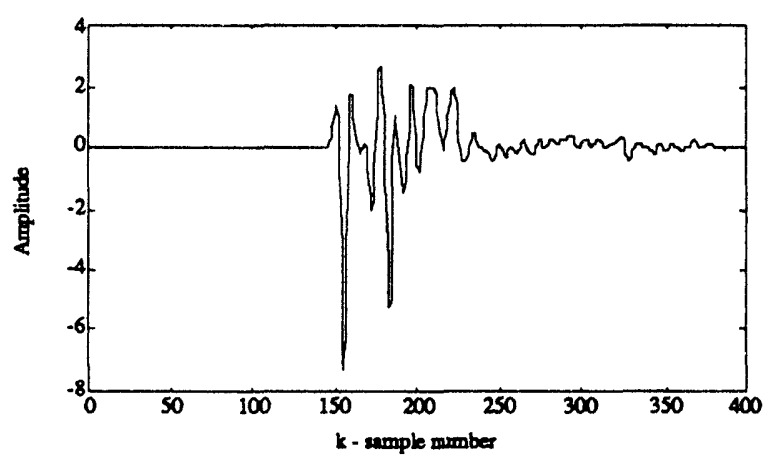

(b)

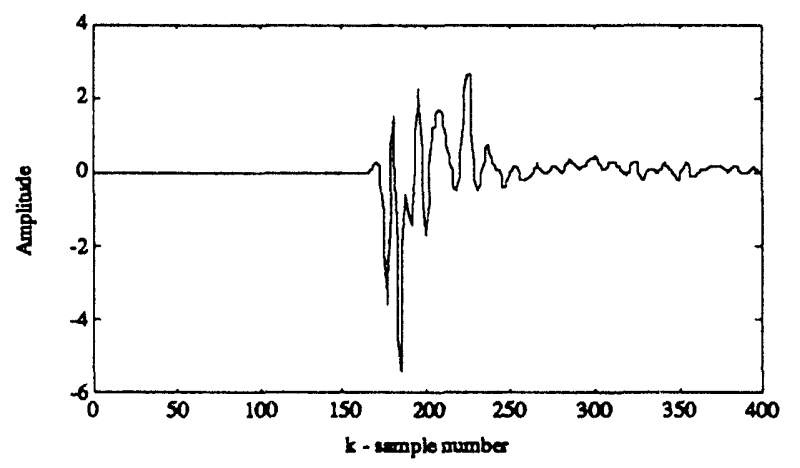

(b) 


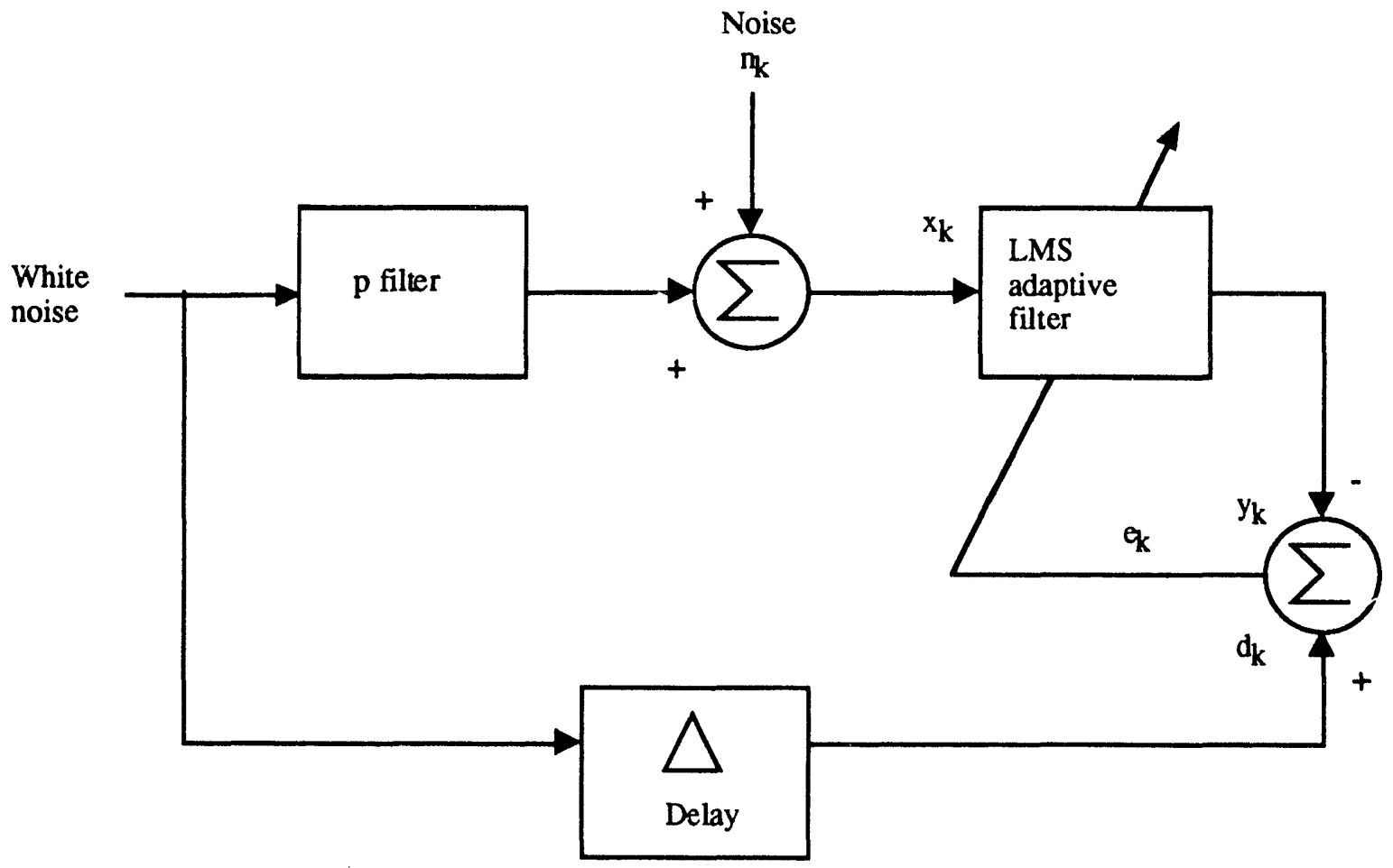

Figure 8. Delayed adaptive inverse model.

\subsection{Adaptive Filter Configuration}

The inverse model adaptive filter to determine target location and identification (void or rebar) is shown in Figure 8. [4] This filter is used to obtain a set of weights that represents the inverse-impulse response for a single void in the concrete. Once these weights are determined, the three-target data without the coupling pulse is convolved with the weights determined by the adaptive filter. In an example that uses a specific receiver position, a set of weights for position 1 is determined by the adaptive filter, using the waveform in Figure 5a (i.e., single-target data without the coupling pulse). These weights are convolved with the data from Figure $5 b$ to generate $a$ filtered signal representing multiple targets. This procedure is applied to all data from the 15 receivers. Next, the synthetic-aperture focusing technique is used to generate a two-dimensional image. The least mean square (LMS) algorithm was used to adapt the inverse model digital filter. The details of operation of the adaptive filter follows.

When the $\Delta$ delay and the noise $n_{k}$ are zero in the ideal inverse model filter of Figure 8 , the internal weights of the adaptive filter adjust and converge so the expected value of the error squared equals zero, i.e., $E\left[e^{2}\right]=0$. In other words, the average of $e_{k}{ }^{2}$ approaches zero as $y_{k}$ matches $d_{k}$. After convergence, the $p$ filter inverse is generated and contained in the weights of the LMS Adaptive Filter block (Figure 8). The inverse-impulse response of the $p$ filter is generated and contained in these weights. Convolution of these weights with the three-target data (without the coupling pulse) generates the filtered signal. The $p$ filter in Figure 8 actually contained a windowed version of single-target data. For example, only 80 samples $(171-250)$ were used from the signal in Figure 5a. This decision was made to reduce computation time and keep the filter from adapting unnecessarily to zeros in the data (see Figure 5a). 
The noise $n_{k}$ in Figure 8 represents plant noise that is present in the adaptive filter but not available to the summation of $y_{k}$ and $d_{k}$. Because $n_{k}$ will cause $y_{k}$ to be noisy, the meansquare error will also increase as $n_{k}$ increases; thus, the inverse model of the $p$ filter is affected, as well as the cleanliness of the filtered three-target data.

The delay in Figure 8 performs two functions: the delay is required to compensate for the internal delays in the $p$ filter and the LMS adaptive filter; however, the $\Delta$ delay is primarily needed when the $p$ filter contains a nonminimum phase signal (i.e., its zeros are outside the z-domain unit circle). Without the delay, the inverse model generated by the adaptive filter would contain poles outside the unit circle where the region of convergence demands the function to be noncausal to meet stability requirements. The delay then generates an approximate causal response from a noncausal sequence. Consequently, the delay also causes the signal generated by the convolution of the weights with the three-target data to be delayed by a $\Delta$ delay amount .

The following calculations were determined for position 1 data, although the same general results can be applied to the other fourteen receivers. The adaptive gain constant $\mu$ and number of weights $(L+1)$ were maintained at the same values for the adaptation of each receiver position. An arbitrary 5\% misadjustment $(M)$ was selected for the inverse model adaptive filter; $M$ is defined as the excess mean-square error (MSE) divided by the minimum MSE. Generally, the MSE is also equal to the $E\left[e^{2}\right]$. Thus the formulation for misadjustment is:

$$
M=\frac{\mathrm{MSE}_{\text {excess }}}{\mathrm{MSE}_{\text {min }}}
$$

which is also designated as:

$M=\mu \cdot \operatorname{tr}[R]$

where $\mu$ is the adaptive gain constant and $\operatorname{tr}[R]$ is the trace of the input auto-correlation matrix, $R$. Because $\operatorname{tr}[R]$ is defined as:
$\operatorname{tr}[R]=(L+1) \cdot$ signal power

where $(L+1)$ is the total number of weights in the adaptive filter, and the signal power is determined by the variance of the signal [5] $x_{k}$ then:

$$
\mu=\frac{M}{(L+1) \cdot(\text { signal power })}
$$

The parameters for position 1 were:

- Variance $=3.4618$.

- $L+1=200$.

- $M=5 \%$.

With these values, $\mu$ is calculated to be .00007 . If we allow $\mu$ to be .0001 and let

$$
(L+1)=200
$$

then misadjustment becomes $6.92 \%$, an acceptable level. The time constant of the learning curve for the filter is given by:

$$
\tau_{\text {use }}-\frac{L+1}{4 \cdot \mu \cdot \operatorname{tr}[R]}=722 \text { iterations }
$$

Assuming the filter totally adapts in five time constants, the MSE learning curve will take approximately 3610 iterations to reach the minimum level. If we consider input to the inverse model to be white noise, then we can statistically approximate the power of the white noise as .0833 . MSE ${ }_{\min }$ is given by:

$$
\mathrm{MSE}_{\min }=\frac{p}{p+1}=.0769
$$

where $p$ is the power of the white noise. The following section examines the inverse model adaptive-filter results. 


\subsection{Adaptive Filter Results}

If the results are presented with waveforms obtained from receiver 1 (supplemented with receivers 8 and 15) we can fully describe the outcome of the adaptive filter by displaying specific waveforms. The contents of the $p$ filter are simply a windowed version of Figure $5 \mathrm{a}$, the single void data with the coupling pulse removed. Receiver 8 would contain a windowed version of Figure $6 \mathrm{a}$ and receiver 15, that of Figure 7a. Using the configuration of Figure 8 with an arbitrary delay of 100 samples and the adaptive filter containing 200 weights, Figure 9a was obtained as the inverse-impulse response of the $p$ filter for receiver 1. Figures $9 b$ and $9 c$ show the results for receivers 8 and 15 , respectively.

As a technique to demonstrate the success of the adaptive filter and show the validity of the inverse model, we convolved the contents of the $p$ filter with the weights determined by the adaptive filter. The resclts of the convolution should present an impulse-shape waveform, and this result is verified in Figures 10a, 10b, and 10c. Note that the impulse has a different location for each receiver position. This result is expected because the distance between the void and each receiver is different, and the reflection from the void arrives at different times.

The mean-square error (MSE) was approximated by averaging the squared error over its 500 nearest neighbors, as shown in Figures $11 \mathrm{a}, \mathrm{b}$, and $\mathrm{c}$ for receivers 1,8 , and 15 , respectively. Although the exact time constant of adaptation is difficult to determine from these waveforms, the constant is within the range calculated from equation (5). Similarly, the amplitude of the MSE is also within the value calculated from equation (7).

Because the inverse model of the system has been determined for each receiver position for a single void, we can now obtain filtered three-target data from the convolution between the weights and the three-target information. For example, we can use the weights in Figures $9 \mathrm{a}, \mathrm{b}$, and $\mathrm{c}$, and convolve these waveforms with Figures $5 \mathrm{~b}, 6 \mathrm{~b}$, and $7 \mathrm{~b}$ to obtain results for receivers 1,8 , and 15 . The resultant signals are shown in Figures 12a, b, and c. The primary goal of this process is target location and identification, i.e., void or rebar.

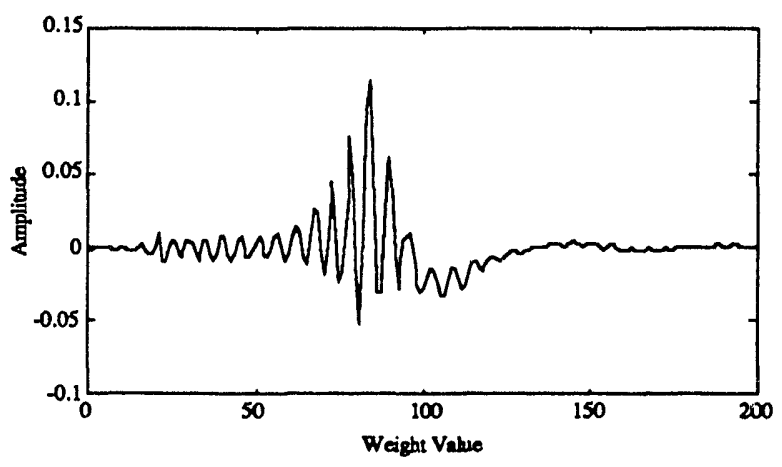

(a)

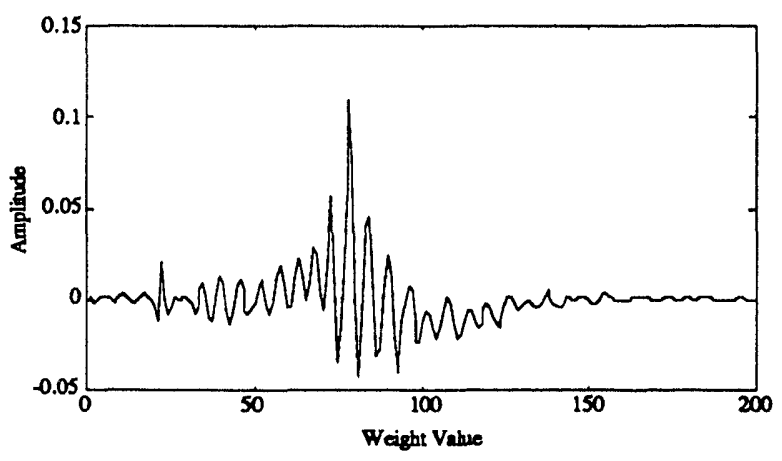

(b)

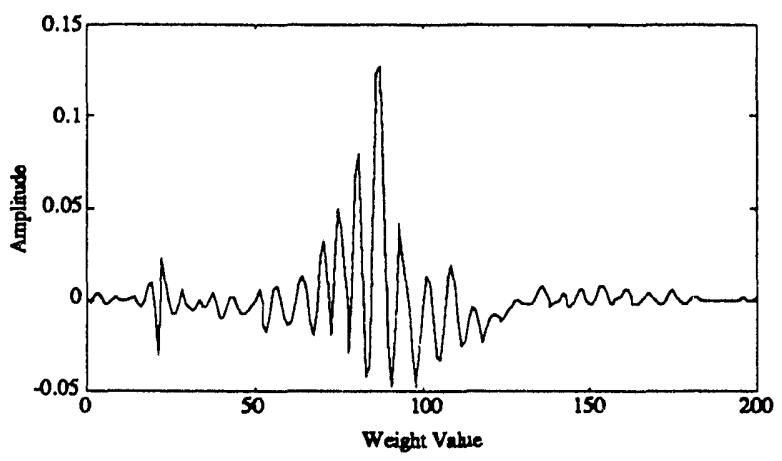

(c)

Figure 9. Inverse model for (a) receiver 1, (b) receiver 8 , and (c) receiver 15. 


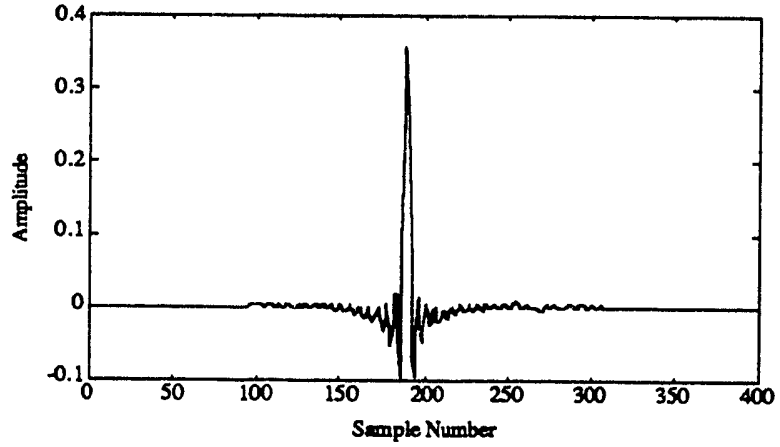

(a)

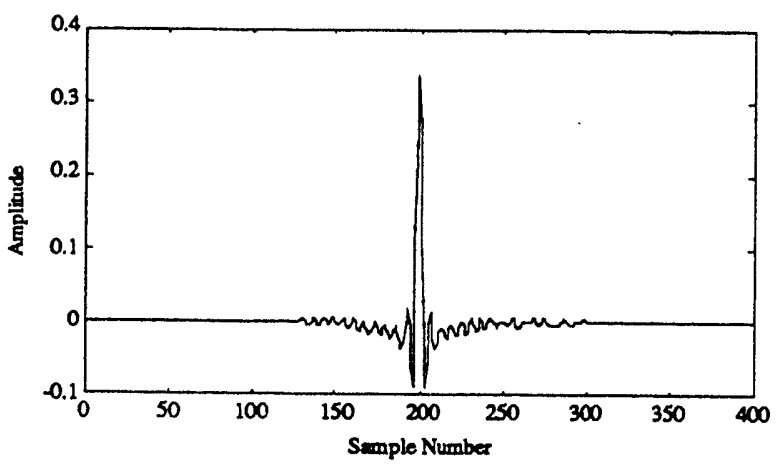

(b)

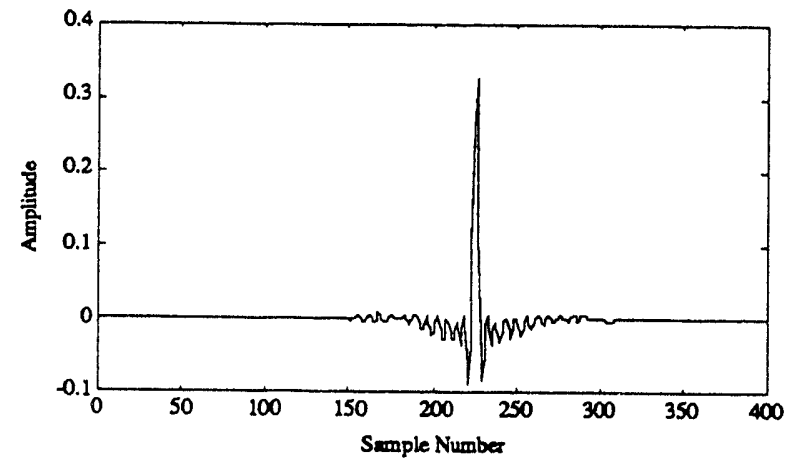

(c)

Figure 10. Convolution $p$ filter and weights for (a) receiver $1,(b)$ receiver 8 , and (c) receiver 15.

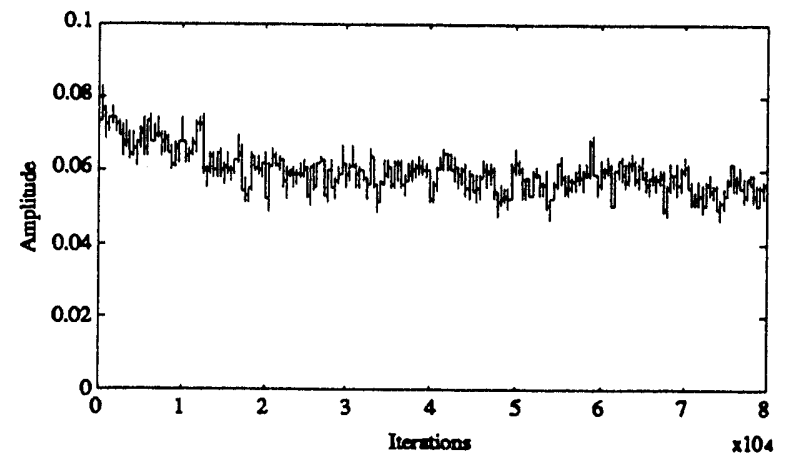

(a)

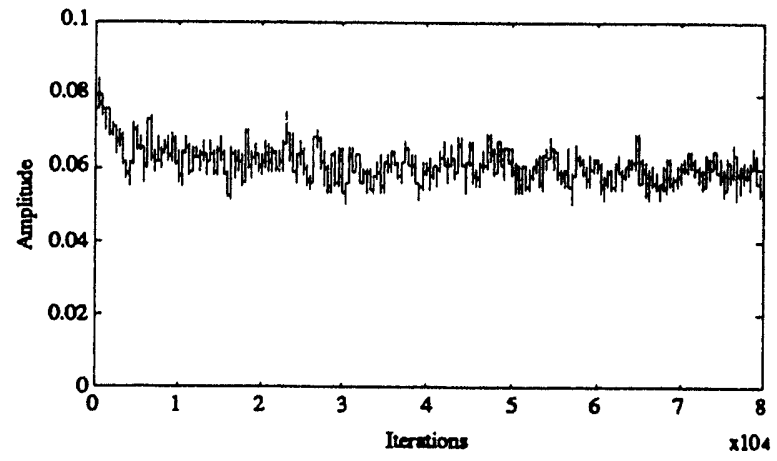

(b)

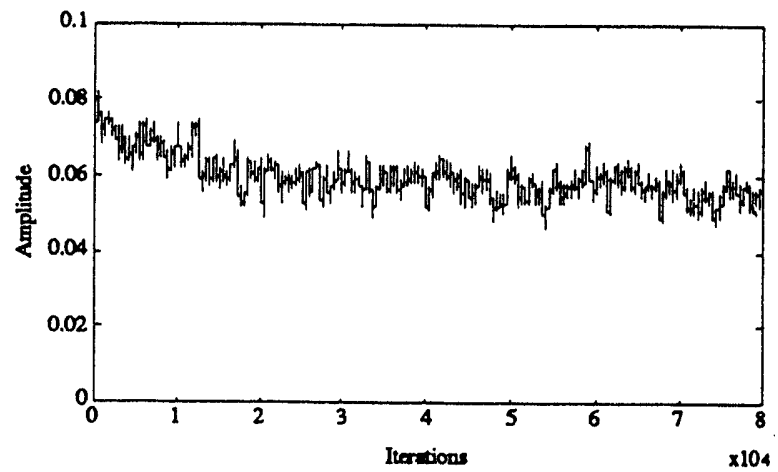

(c)

Figure 11. Mean-square error for the adaptive filter for (a) receiver 1 , (b) receiver 8 , and (c) receiver 15. 
As shown by Figure 12a, we can predict that there are two rebars and a single void in the concrete. The two rebars are located at sample number 160 and 205; the void is located at sample number 190 . The data for Figure $12 \mathrm{~b}$ contains the same information as Figure 12a, except that the receiver location is now in position 8 . The two rebars are located at positions 160 and 180, and the void is at 195 . The data from Figure 12c distinctly shows rebar locations; however, the void location is more difficult to determine. The void is located at sample number 225 . The extra oscillations of Figure $12 \mathrm{c}$ are created by the close proximity of rebars and the resultant convolution with the weights. These three waveforms show the complexity of the of the signals and some of the problems encountered in trying to determine target location and identification. However, if we compare signals from Figure $12 \mathrm{a}, \mathrm{b}$, and $\mathrm{c}$ with Figures $6 \mathrm{a}, \mathrm{b}$, and $\mathrm{c}$, we can recognize signal improvement resulting in better target identification and location.

\subsection{Post Adaptive Filter Processing}

To generate the impulse-shaped waveforms of Figures 10a, b, and c, the lower frequency content of single-void data required amplification. This amplification is clearly shown by examining the frequency spectrum of three-target data without the coupling pulse, the frequency spectrum of the weights from the adaptive filter, and the frequency spectrum of the filtered three-target data. Th? frequency transforms of signals from receiver 1 for Figures $5 b, 9 a$, and 12a are shown in Figures $13 \mathrm{a}, \mathrm{b}$, and $\mathrm{c}$, respectively [6]. Figure $13 \mathrm{c}$ clearly shows that the lower frequency spectrum has been amplified by the adaptive filter. By amplifying these lower frequencies, we also generate the low-frequency oscillations shown in Figures 12a, b, and c. Theses lowfrequency oscillations are particularly significant toward the end of these waveforms. Because these frequencies are in phase and because the synthetic aperture focusing technique applies the values obtained by the adaptive filter across a two-dimension image plane, the amplitudes of these lower frequencies increase in magnitude. The focusing technique generates a white smear in the two-dimensional image, as shown by the bottom of Figure 14a. If we apply a modified Blackman window [7],[8] to reduce the magnitude of these lower frequencies from the

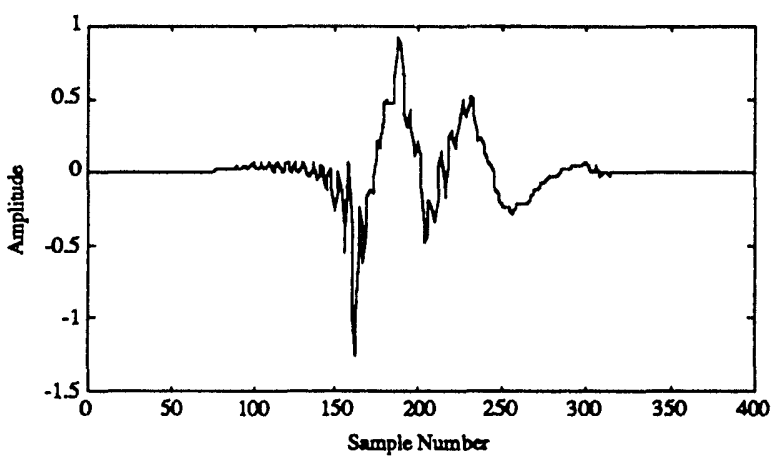

Figure 12a. Filtered three-target data for receiver 1 .

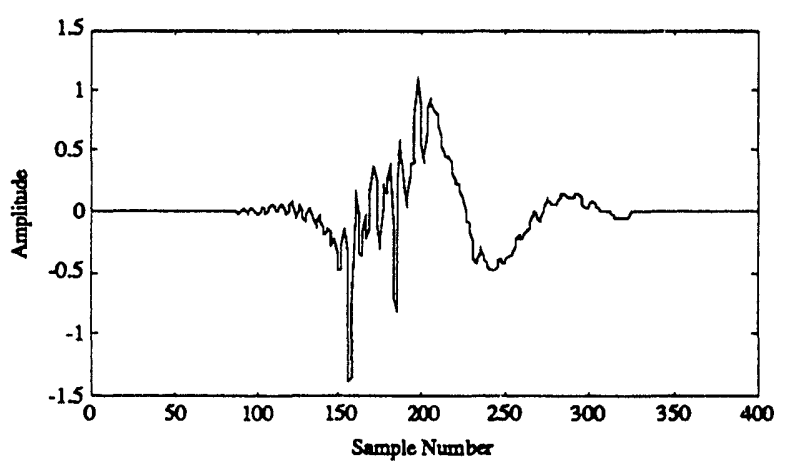

Figure 12b. Filtered three-target data for receiver 8.

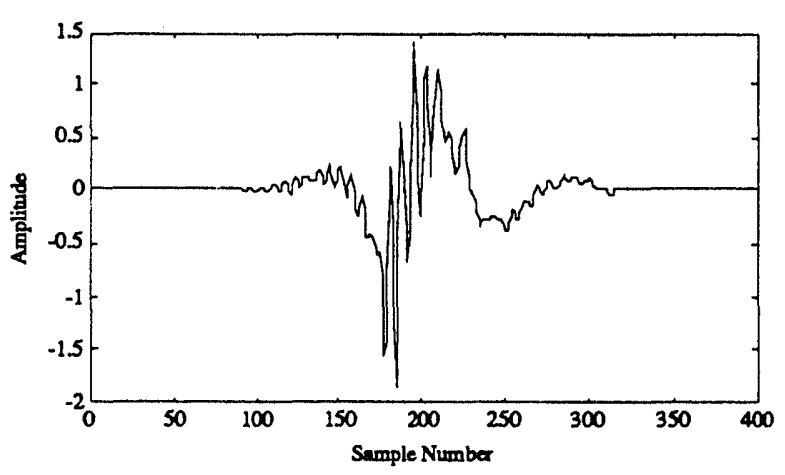

Figure 12c. Filtered three-target data for receiver 15. 


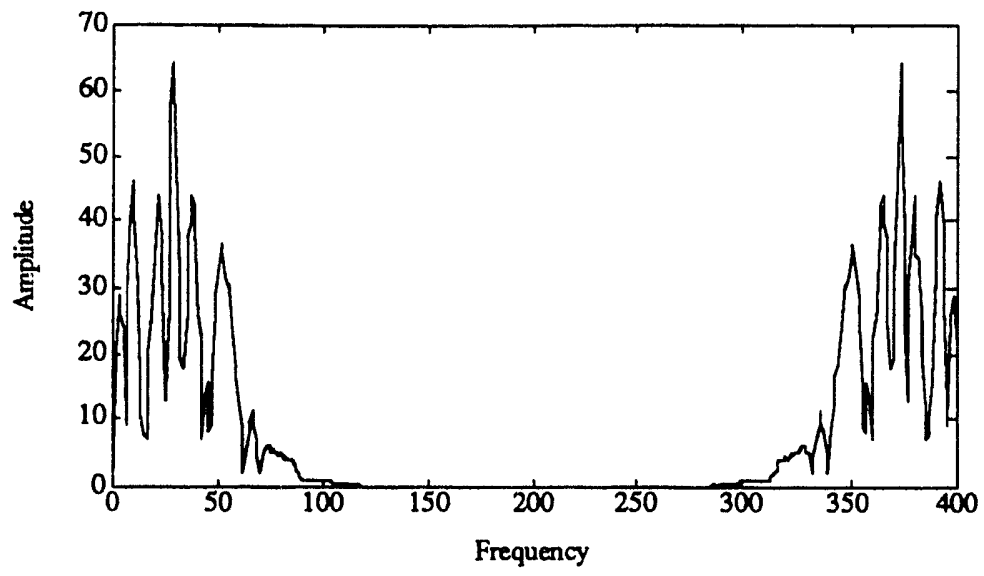

Figure 13a. Frequency transform of three target data for position 1 (without coupling pulse).

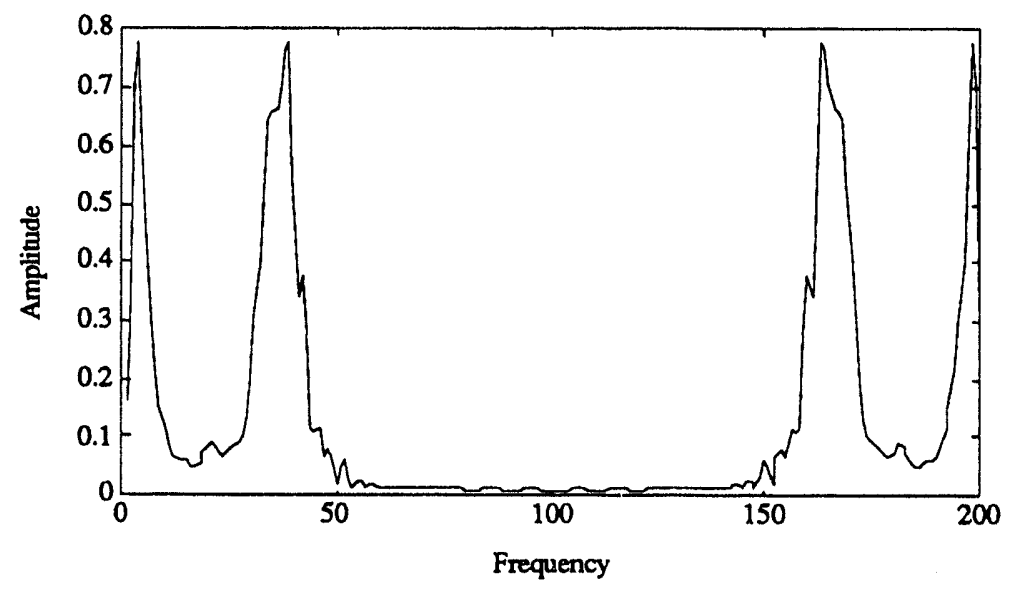

Figure 13b. Frequency transform of weights for position 1 .

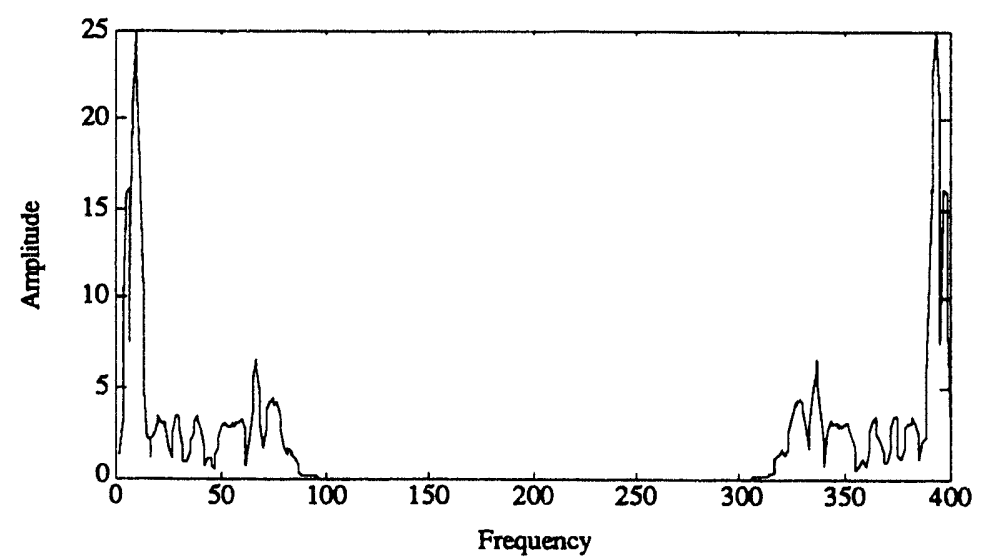

Figure 13c. Frequency transform of filtered three target data for position 1. 


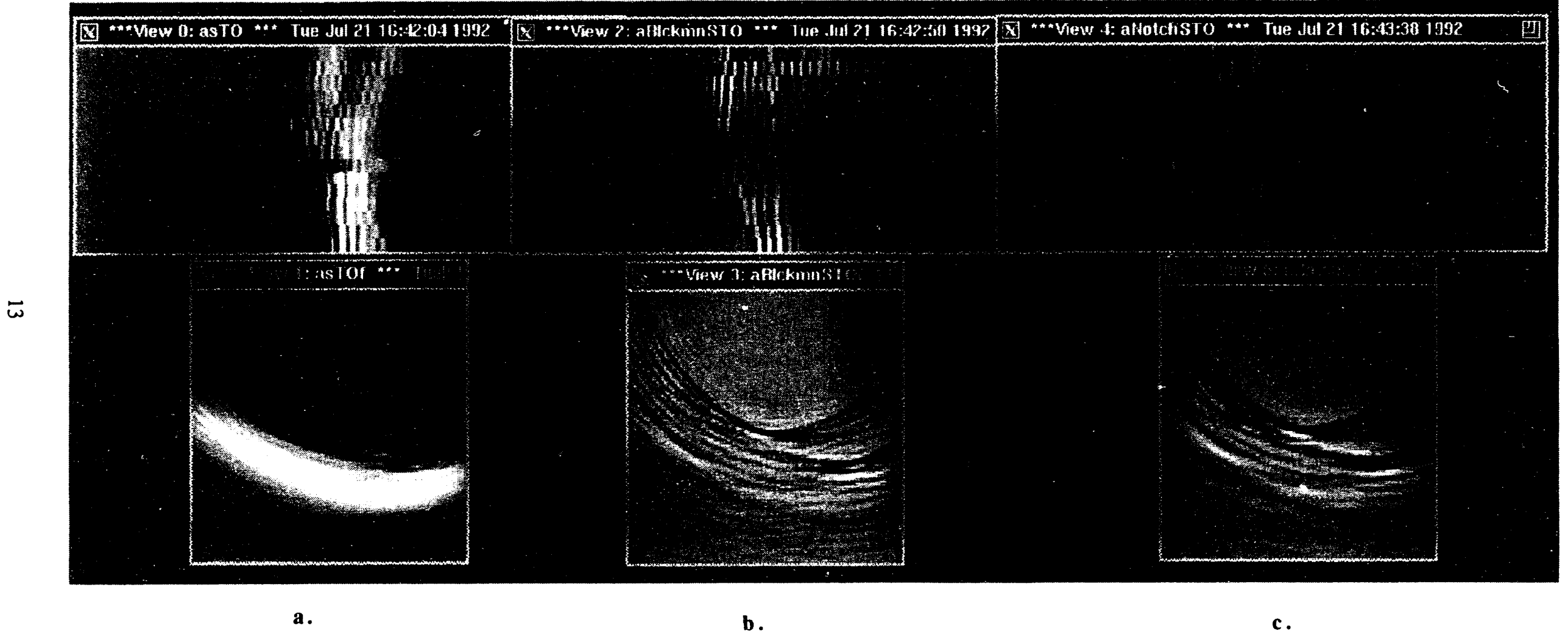

Figure 14: Pre-focused (top) and focused (bottom) three target data

a. Output from adaptive filter

b. Output from modified Blackman window

c. Output from adaptive notch filter 


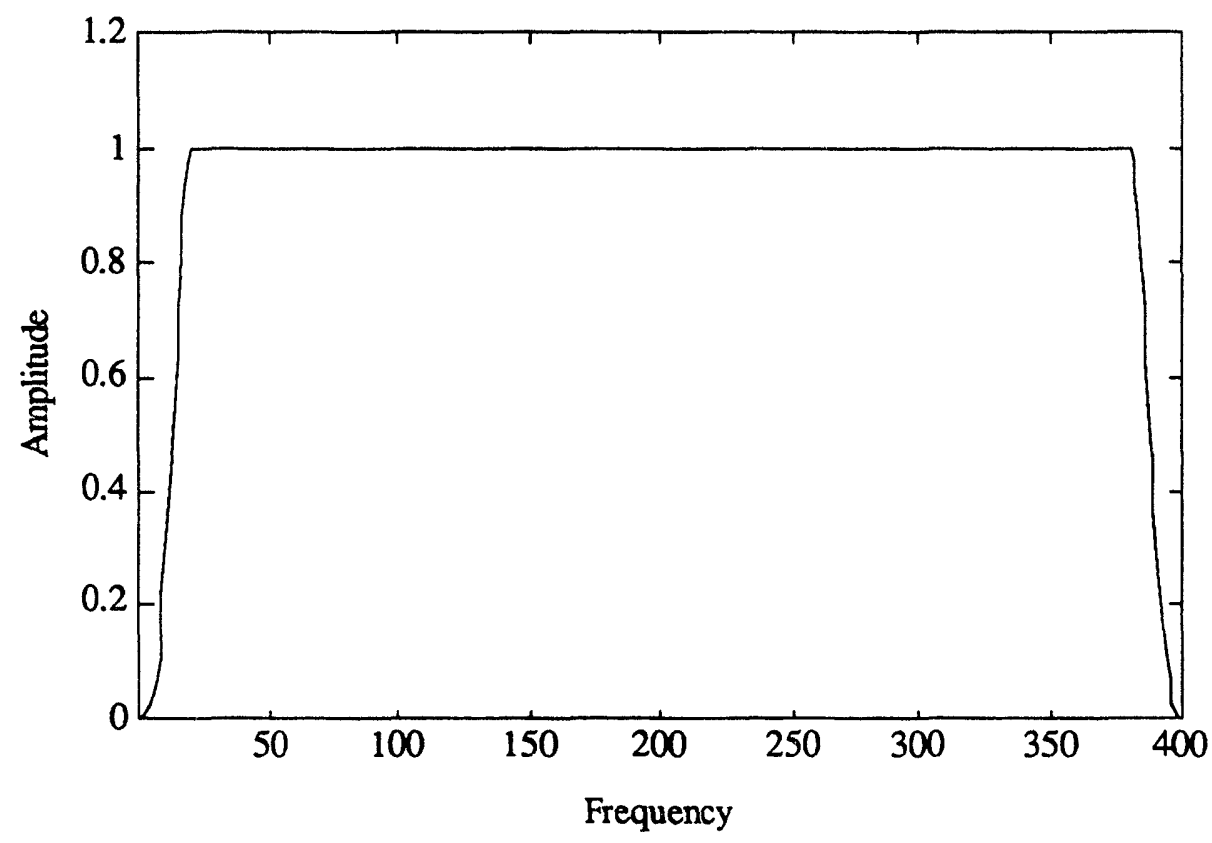

Figure 15. Modified Blackman window.

filtered three-target data, the bottom of Figure $14 \mathrm{~b}$ becomes the resultant two-dimensional image. The modified Blackman window consisted of a typical 40-sample Blackman window with 360 "ones" inserted between sample numbers 20 and 21 . Thus, the modified Blackman window contained 400 samples with a smooth transition between the unequal amplitudes, as seen in Figure 15. The same modified Blackman window was applied equally to all filtered three-target data. The tops of Figures $14 a-c$ show the data before the twodimensional synthetic-aperture focusing technique was applied to the data. The signals from receiver 1 are at the upper most part of these figures.

An adaptive notch filter was used next on data generated by the modified Blackman window. The notch filter was used to diminish the low frequency oscillations in Figure 12a-c while trying to maintain as much the target information as possible. Like the modified Blackman window, the notch filter was applied evenly for all the receiver data. An adaptive notch filter was selected rather than conventional notch filters, such as an elliptic filter, ${ }^{[9]}$ for several reasons. Primarily, the adaptive notch filter did not generate as much ringing as the other filters did. In fact, at times the ringing generated by the nonadaptive notch-filter data was greater than the original signal.

Moreover, the adaptive notch filter permitted easy control over the frequency bandwidth and provided an infinite null at the notch frequency. The parameters for the adaptive filter were:

$$
\omega_{o}=2 \cdot \pi \cdot f \quad(\mathrm{rad})
$$

where $f$ is the frequency to be removed. The frequency for this filter was $0.15 \mathrm{rad}$. The adaptive time constant, $\mu$, was chosen as 0.05 $\mathrm{rad}$, where $\mu$ determines the bandwidth (BW) from:

$$
B W=2 \cdot \mu \quad(\mathrm{rad})
$$




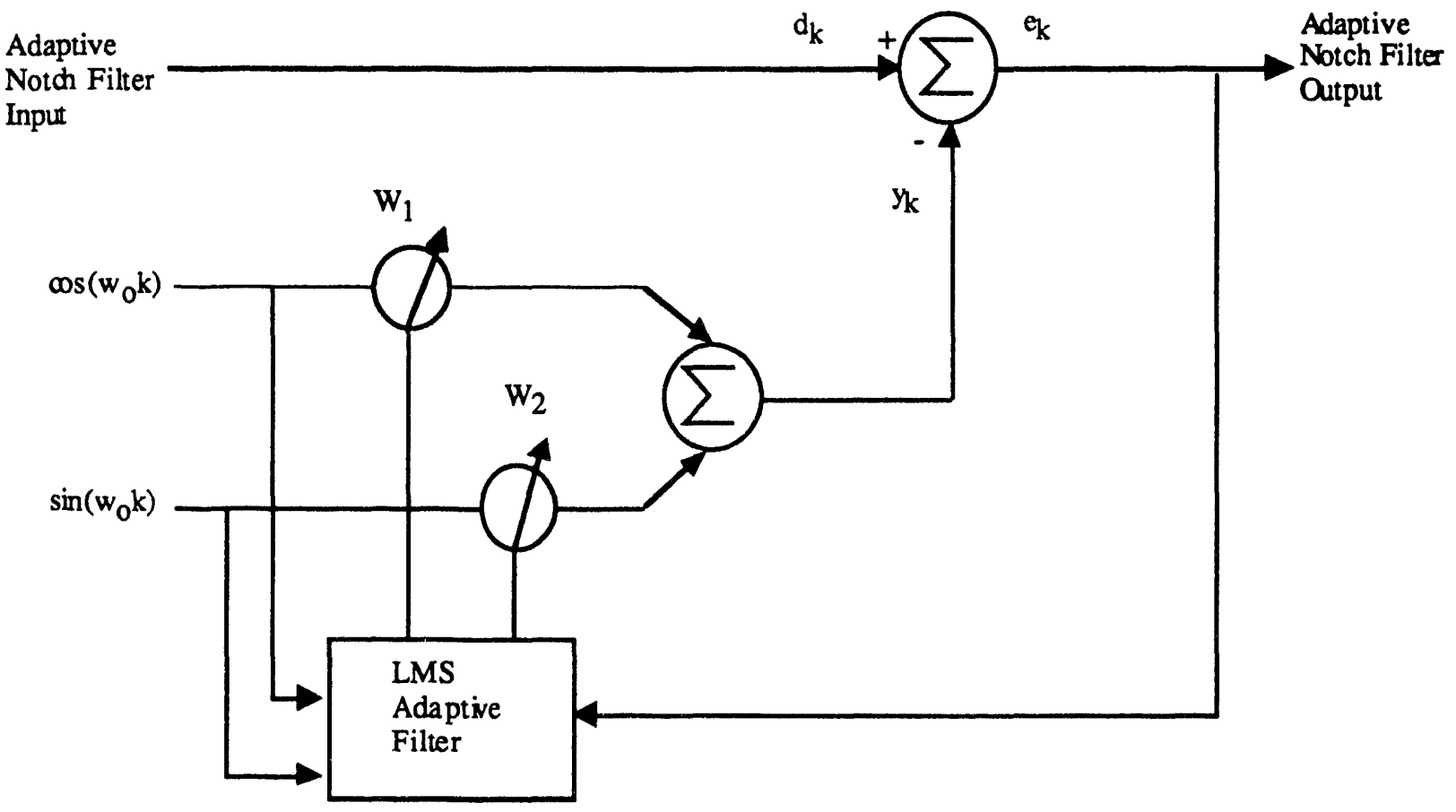

Figure 16. Adaptive notch filter.

Because 400 frequency sample points are used, the frequency and bandwidth must be scaled appropriately to obtain:

$\mathrm{f}=64 \quad \mathrm{~Hz} \quad$ and,

$\mathrm{BW} \approx 64 \mathrm{~Hz}$ at $-3 \mathrm{db}$.

After the 15 signals were applied to the adaptive notch filter, the data were processed through the synthetic-focusing technique to generate the two-dimensional image of Figure 14c. The single void and the two rebar are shown in this figure; the rebars are dark areas and the void is the lighter area. The "cat whiskers" type artifacts in this picture are caused by the limited number of receivers. These artifacts depend on the number of receivers and should reduce in intensity as the number of receivers is increased. (Section 7 details the signal-to-artifact calculations as a result of using the synthetic aperture image focusing technique.) The uppermost portion of Figure 14 exhibits the time domain filtered data before application of the focusing technique. The curvature of the light and dark strips in the top portions is caused by the difference in distance between each target and each receiver. The $y$ axis in this figure was magnified by 5 to increase the width of the waveforms. 


\section{Variable Size Single Target}

\section{in Dispersive Concrete}

\subsection{Modeled Data Configuration:}

The configuration of the single-target dispersive model is similar to Figure 1, i.e., the model had 15 receivers and one transmitter. However, instead of multiple targets, the modeled data for this configuration contained a single void of changing size inside dispersive concrete with aggregate. The void ranged in size from $5 \mathrm{~mm}$ to $75 \mathrm{~mm}$ in $5-\mathrm{mm}$ steps, and the void was modeled as if it were filled with dry air. The front surface of the void was kept at a depth of $91 \mathrm{~mm}$ as the void size changed. The aggregate is modeled with a dielectric constant of 4.3 and an individual size of $4 \mathrm{~mm}$ by $5 \mathrm{~mm}$. Because cement is dispersive, its dielectric properties are a function of frequencies transmitting through it and thus are not a constant. The transmitted pulse had a 4-ns pulse peak and a 100-ps FWHM. Like the multiple-target data, these data were also supplied with a no-target data set. The set included a waveform for each receiver without the reflection caused by the void. Removing the coupling pulse required a simple subtraction between the data of different size voids and the no-void data. As the model of Figure 1 suggests, the transmitter and receivers were modeled as if they were inside the concrete pad. Therefore, the initial reflection pulse from the front surface of the concrete was not considered.

The objective of this section was to determine a standard set of weights from a given receiver position and void size, apply these weights to other receiver positions and other void sizes, and then produce target images where compensation for dispersion was considered. In this experiment, receiver 1 and the data for the $40-\mathrm{mm}$ void were selected to generate the standard set of weights. The weights could be generated off-line so that the only field computation required would be convolving the weights with the data recorded. These weights would then compensate for concrete dispersion for different size voids at a given depth. Because generating adaptive-filter weights requires a significant amount of computation time, the weights could be determined off-line without significantly affecting imare production in the field; final convolution with field data does not require much computation time. Because the weights contain the inverse-impulse response of the concrete for a given void size and position, using the standard weights on other void sizes and other receiver positions will not produce ideal images. However, the following sections will show that the images are complete enough to visualize the positions for the different size voids.

\subsection{Adaptive-Filter Configuration}

The adaptive filter used to determine the inverse-impulse response for a single void in dispersive cement is identical to Figure 8, its operation is described in Section 2.2.

Parameters selected for this filter are similar to the multiple-target data, i.e., the number of weights and the delay are respectively:

$$
\begin{aligned}
& L+1=200, \\
& D=100 .
\end{aligned}
$$


The adaptive time constant and the number of iterations to adapt the filter are different then those from the previous section. These parameters were changed because the window selected to adapt the filter was increased, and the data included dispersive effects. These parameters are:

$$
\begin{aligned}
& \mu=1 e-8, \\
& \text { Iter }=50000 .
\end{aligned}
$$

With the above parameters and with the variance of the $x$ matrix equal to 6038.2 , the misadjustment was calculated as $1.21 \%$ from equations (2) and (3). Equation (5) gives the leaming curve time constant. Assuming five time constants, full adaptation is reached in approximately 20,700 iterations.

The void size used to determine the standard set of weights was chosen from the $40-\mathrm{mm}$ data receiver position 1 . The waveforms for receiver $i$ are shown in Figures $17 a-c$. Figure 17a includes the coupling pulse in the data for the single void, while Figure $17 \mathrm{~b}$ contains the waveform without the void reflection. To remove the coupling pulse, Figure $17 \mathrm{~b}$ is subtracted from the data in Figure $17 \mathrm{a}$ to generate Figure 17c. The actual waveform used in the adaptation of the digital filter is a reduced version of Figure 17c; instead of 400 samples, the waveform included only 200 samples, 90-289.

Figure 18 shows he standard weights determined by the adaptive filter. To verify the validity of the adaptive filter, the contents of the $p$ filter were convolved with the weights. As expected, the results are demonstrated in the impulse-shaped waveform of Figure 19. Figure 20 shows the waveform of the MSE (once again, determined by averaging the squared error over its 500 nearest neighbors).

\subsection{Adaptive Filter Results and Post Adaptive Filter Processing}

After the set of standard weights were determined from the $40-\mathrm{mm}$ void size, images were produced for several void sizes using these same weights. The purpose of imaging different void sizes in this particular experiment was to see if these weights could be used in a general sense. Also, the purpose was to establish

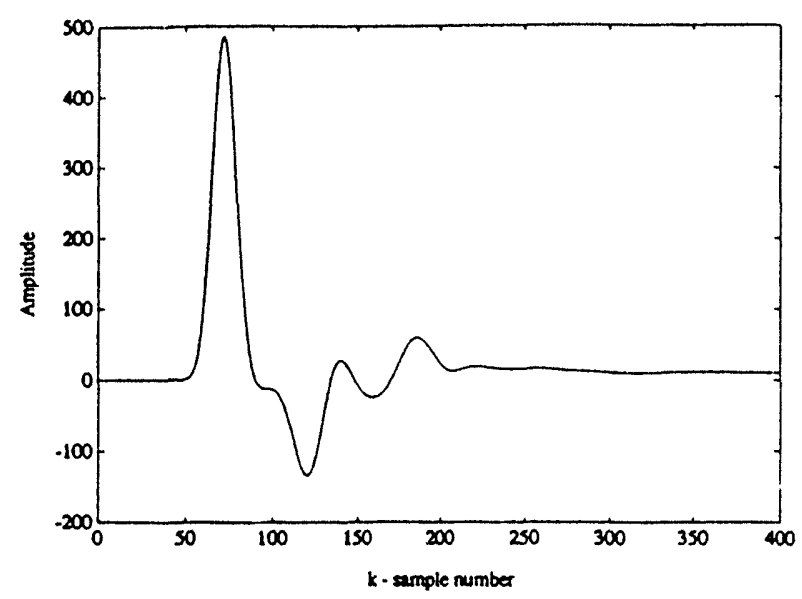

Figure 17a. Single void in dispersive medium with coupling pulse.

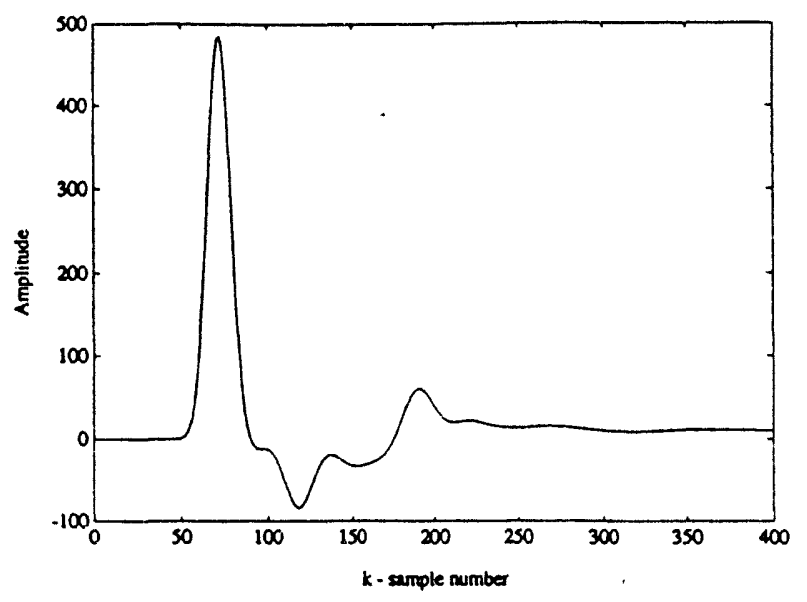

Figure 17b. Received signal without void.

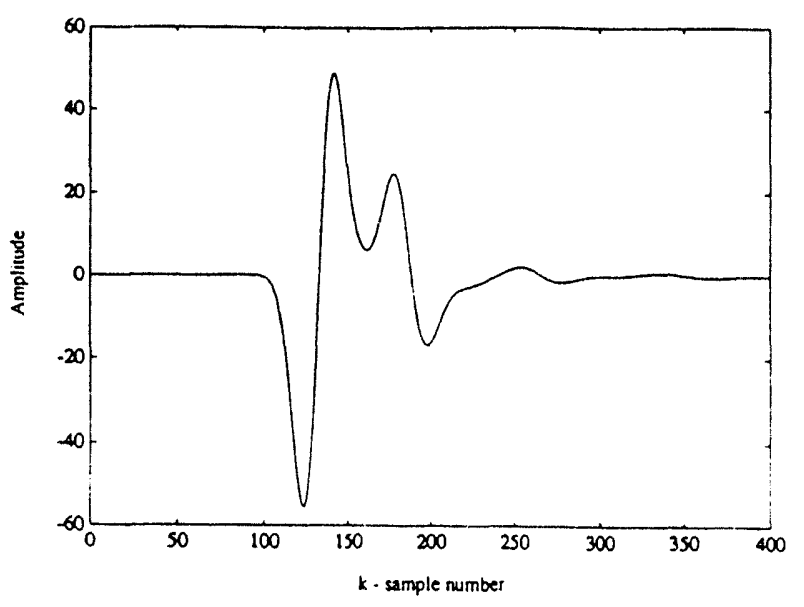

Figure 17c. Void in dispersive medium without coupling pulse 


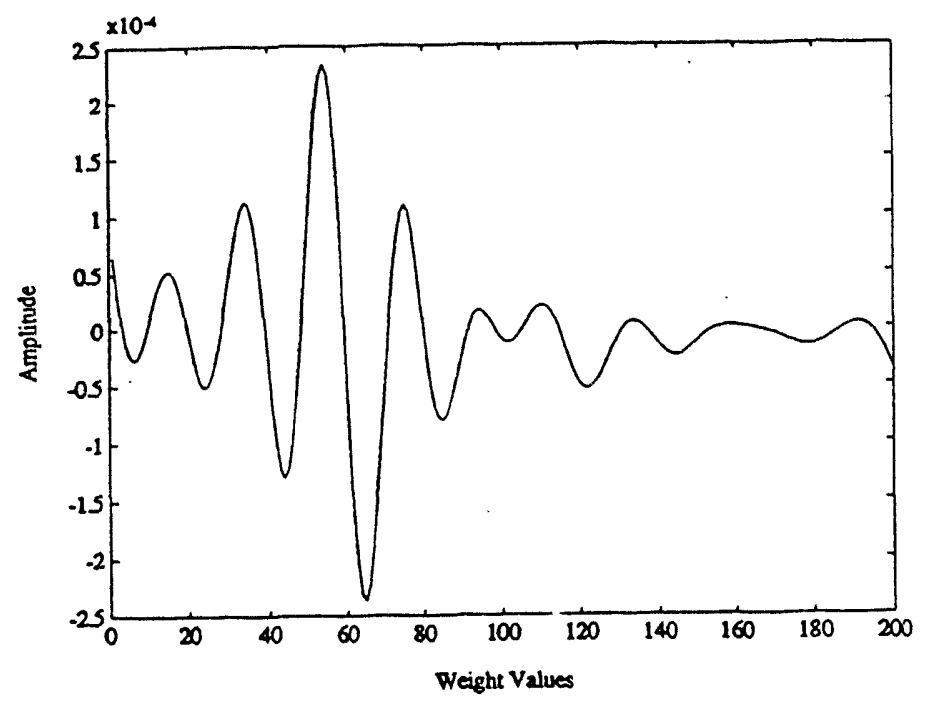

Figure 18. Standard weights for $35 \mathrm{~mm}$ void from receiver 1 .

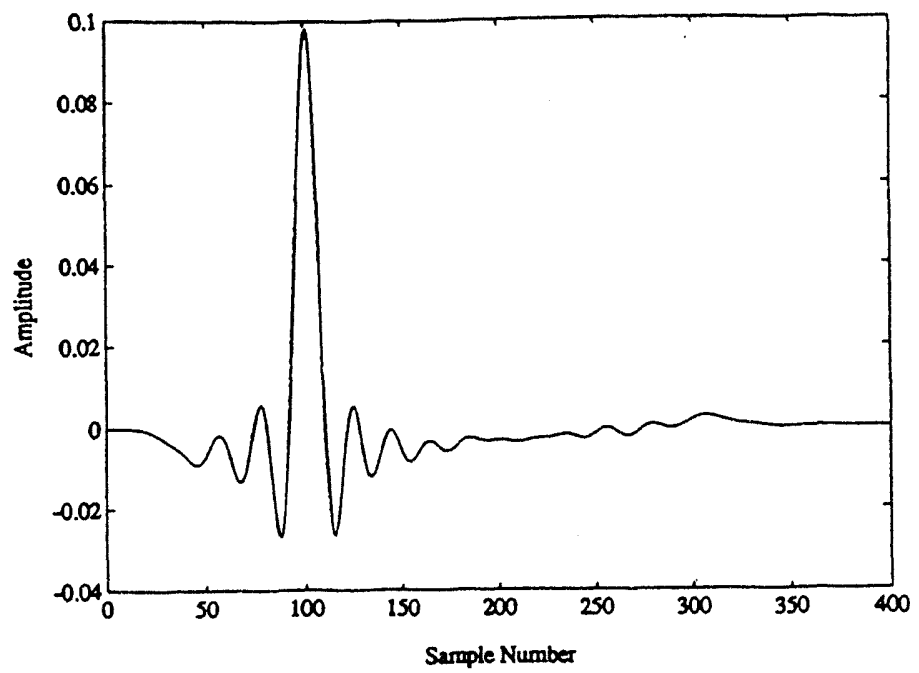

Figure 19. Convolution results of "p" filter and weights.

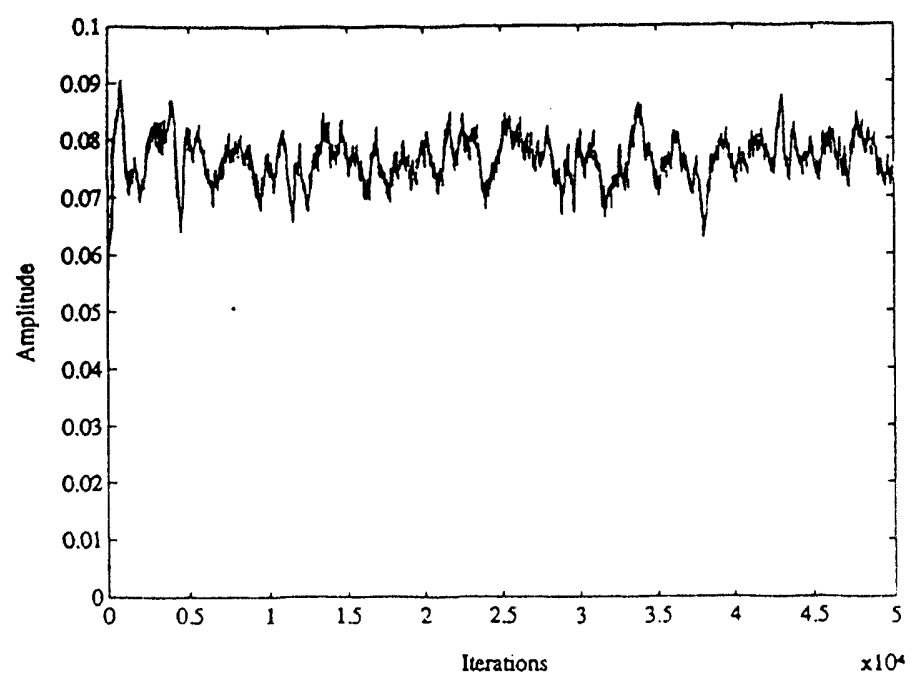

Figure 20. Mean-square error for adaptive filter. 
any limits in which these general set of standard weights could not be used. The standard set of weights were used on the following void sizes: $5 \mathrm{~mm}, 35 \mathrm{~mm}, 55 \mathrm{~mm}$ and $75 \mathrm{~mm}$. The images are shown in Figures 2lb, 22b, 23b, and 24b, respectively. Figures 21a, 22a, 23a, and 24a display the waveforms before standard weights were applied to the data. In each of these figures, the rectangular figure represents the time domain prefocused data, and the square figure represents the focused data. The bright area signifies the higher amplitude signal and the location of the target. As with multipletarget data, the $y$ axis in the time domain figure was magnified by 5 to increase the width of each waveform. The data for receiver position 1 , is the uppermost signal of the rectangular figure. (The focusing technique will be discussed in detail in Section 6.)

Note that in each time domain figure, the lighter-colored portion of the signal was sharpened after the standard set of weights were applied. In addition, the focused figures where the adaptive filter weights had not been applied, show that the location of the void was further away from the surface of the concrete than they were in reality. This shift is due to the dispersive effects caused by the concrete, which had changed the arrival time of the reflected signal. After the previously generated standard weights were applied to the data, the location of the void in the focused figure was correctly located. This was verified by determining the location of the pixels in the picture of the two-dimension image plane.

The differences in void sizes in Figures $21 \mathrm{~b}, 22 \mathrm{~b}, 23 \mathrm{~b}$, and $24 \mathrm{~b}$ are not notably present. The primary reason is the inadequate crossrange resolution used to obtain the modeled data. The resolution is determined by the width of the synthetic aperture and the properties of the transmitted pulse. In a first-order approximation, the cross-range resolution is nearly $78 \mathrm{~mm}$ and the size of the voids range from $5 \mathrm{~mm}$ to $75 \mathrm{~mm}$. (These calculations are explained in detail in Section 7.)

Determining the most deteriorated image from the void images of Figures $21,22,23$, and
24 is moderately difficult. Any deterioration in these images is caused by using standard weights generated from a different size void. It does appear, however, that the "cat whiskers" in Figure 21 are more apparent than in the other figures. Using this criterion, we can say conservatively that weights generated from a 40-mm void can generate images of voids, ranging from $5 \mathrm{~mm}$ to $75 \mathrm{~mm}$.

Figure 25a-c show the image generated from the data of the $75-\mathrm{mm}$ void, including an additional post-adaptive-filter process introduced in Figure 25c. This process was used to remove the "cat whiskers." The analytic signal ${ }^{[10]}$ component of the data that had been processed with the standard weights was used to remove these artifacts. The analytic signal was used because its magnitude is directly related to the rate of energy arrival and therefore is a good interface location estimator for signals obtained from radar reflections. Since the magnitude of the analytic signal obtains the envelope of the signal that was processed with the adaptive filter weights, the resolution of the image could deteriorate. However, the analytic signal does provide better image resolution than simply applying a low-pass filter and removing the highfrequency components. ${ }^{[11]}$

The analytic signal was obtained by applying a fast Fourier transform (fft) to each receiver post-adaptive-filter signal and then removing the negative frequency components. Subsequent steps required taking the inverse fft of the above signal and obtaining its magnitude by taking the square root of the sum of the real component squared and the imaginary component squared. Because phase information is lost when the magnitude of the signal is taken, current investigations are exploring how to re-obtain this phase information from the real and imaginary components of the analytic signal.

Figures 25a and $\mathrm{b}$ are the same figures presented previously in Figures 24a and $b$ for the $75-\mathrm{mm}$ void. They are shown again only for comparison with the analytic signal results. 


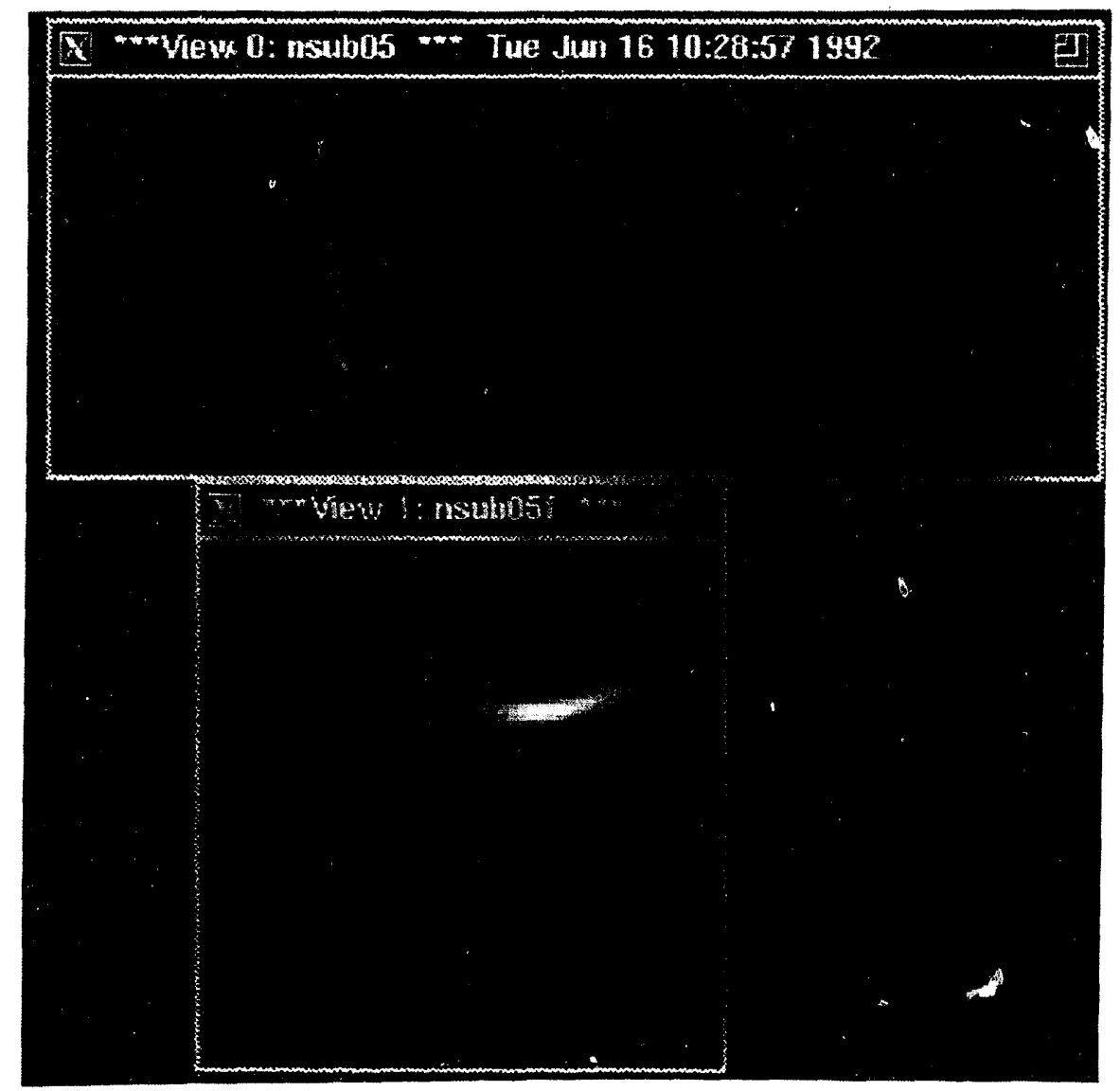

Figure 21a. Image of $5 \mathrm{~mm}$ void before applying adaptive filter weights.

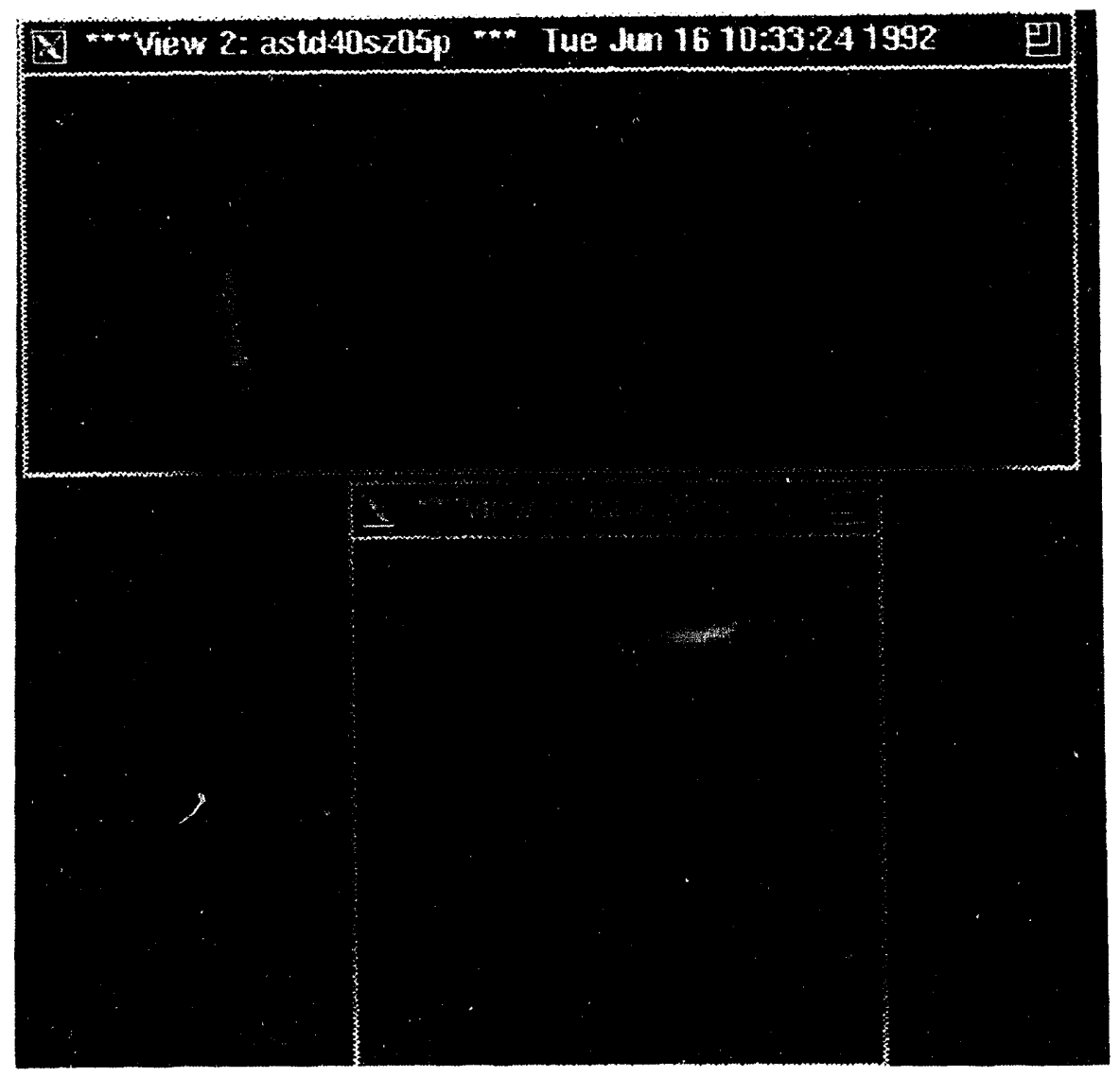

Figure 21b. Image of $5 \mathrm{~mm}$ void post-adaptive-filter weights process. 


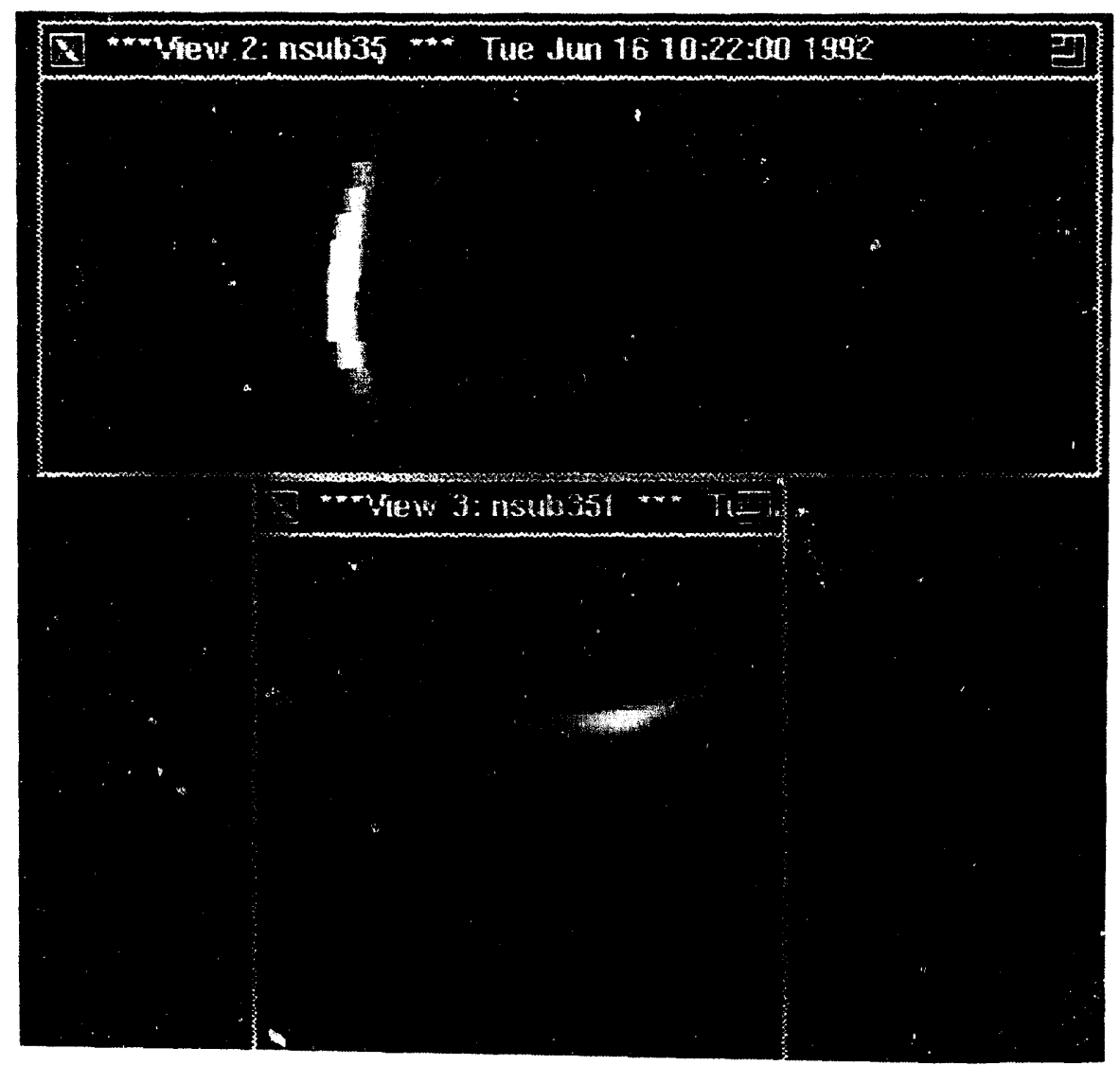

Figure 22a. Image of $35 \mathrm{~mm}$ void before applying adaptive-filter weights.

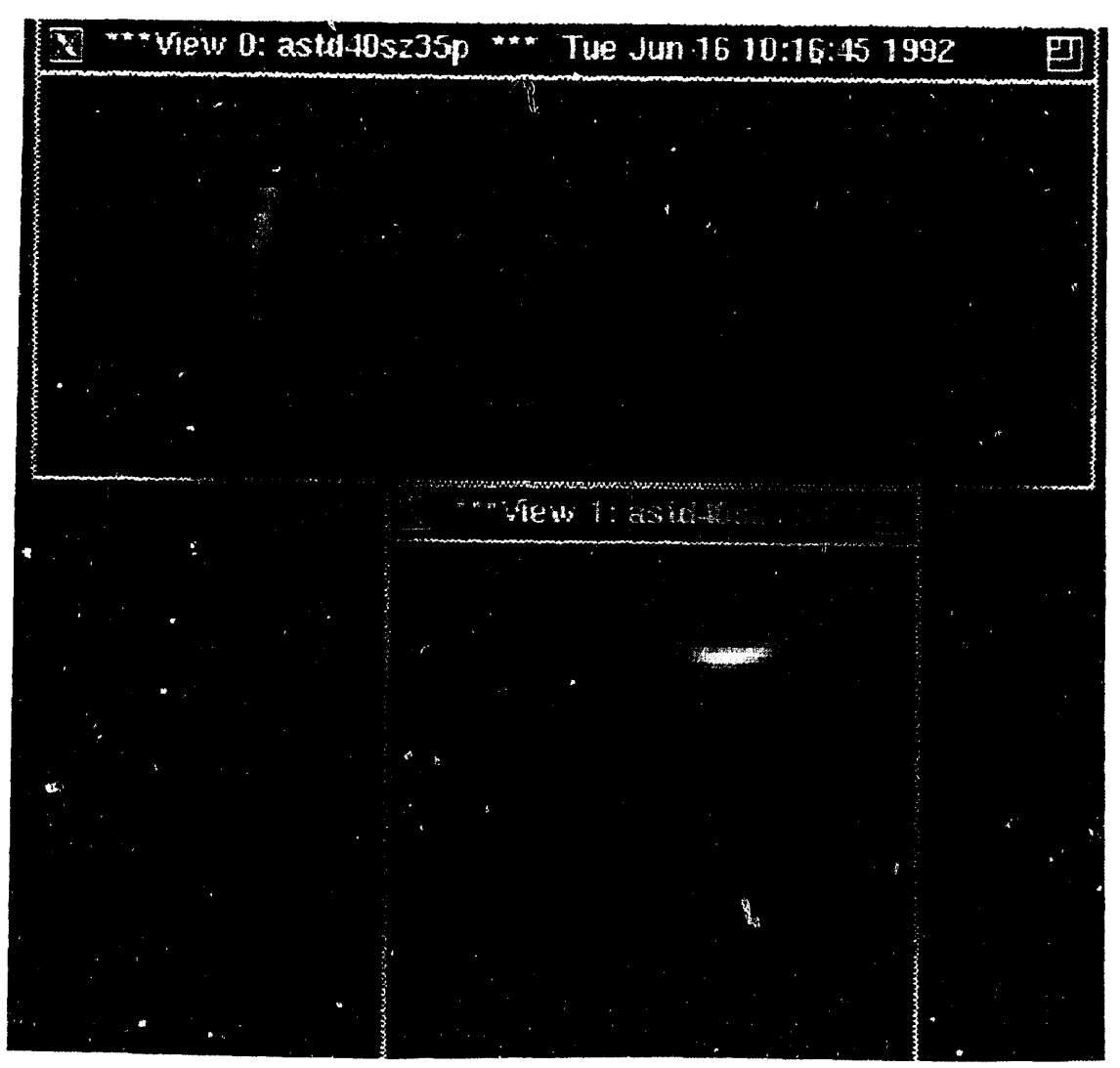

Figure 22b. Image of $35 \mathrm{~mm}$ void post-adaptive-filter weights process. 


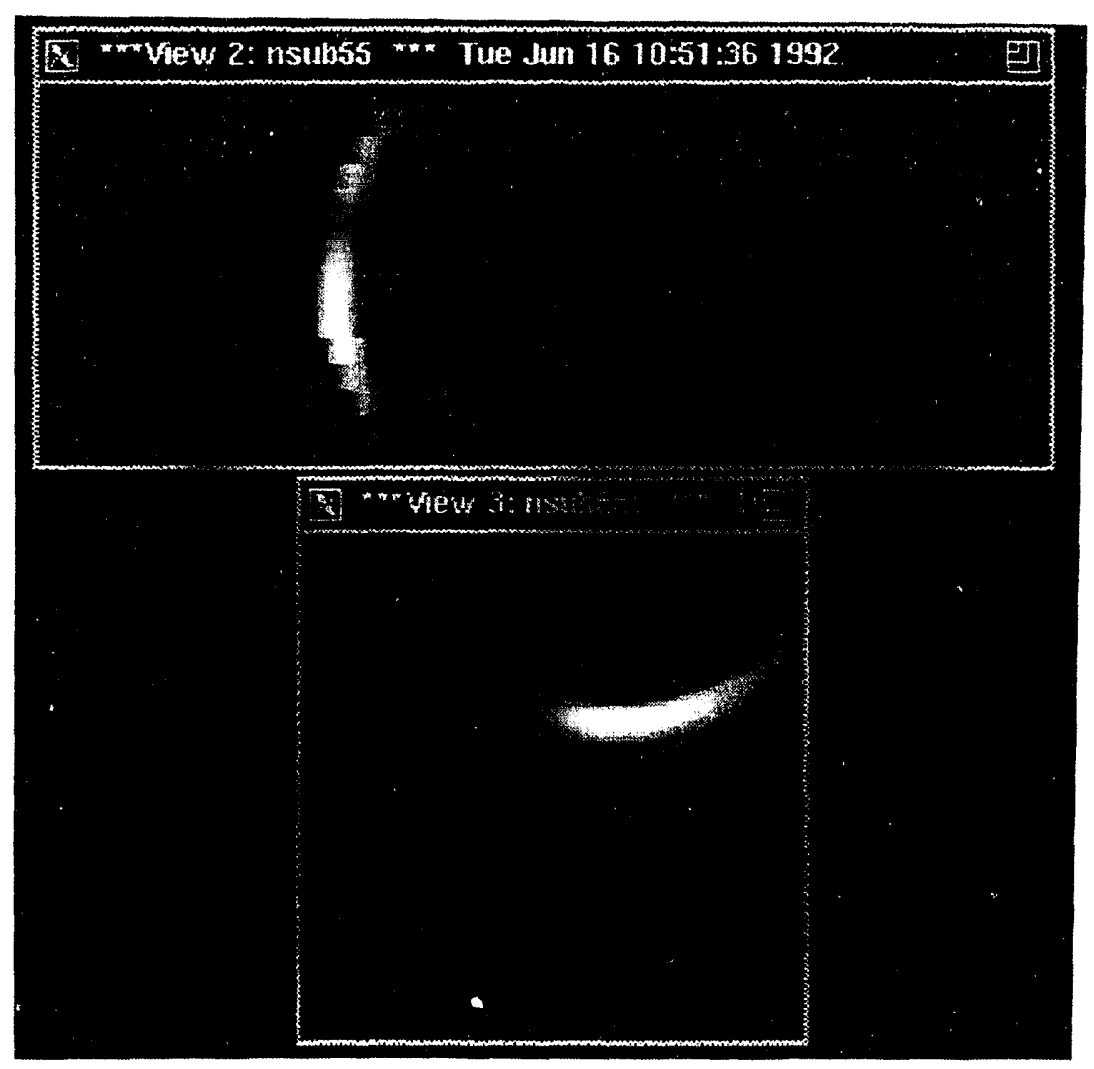

Figure 23a. Image of $55 \mathrm{~mm}$ void before applying adaptive-filter weights.

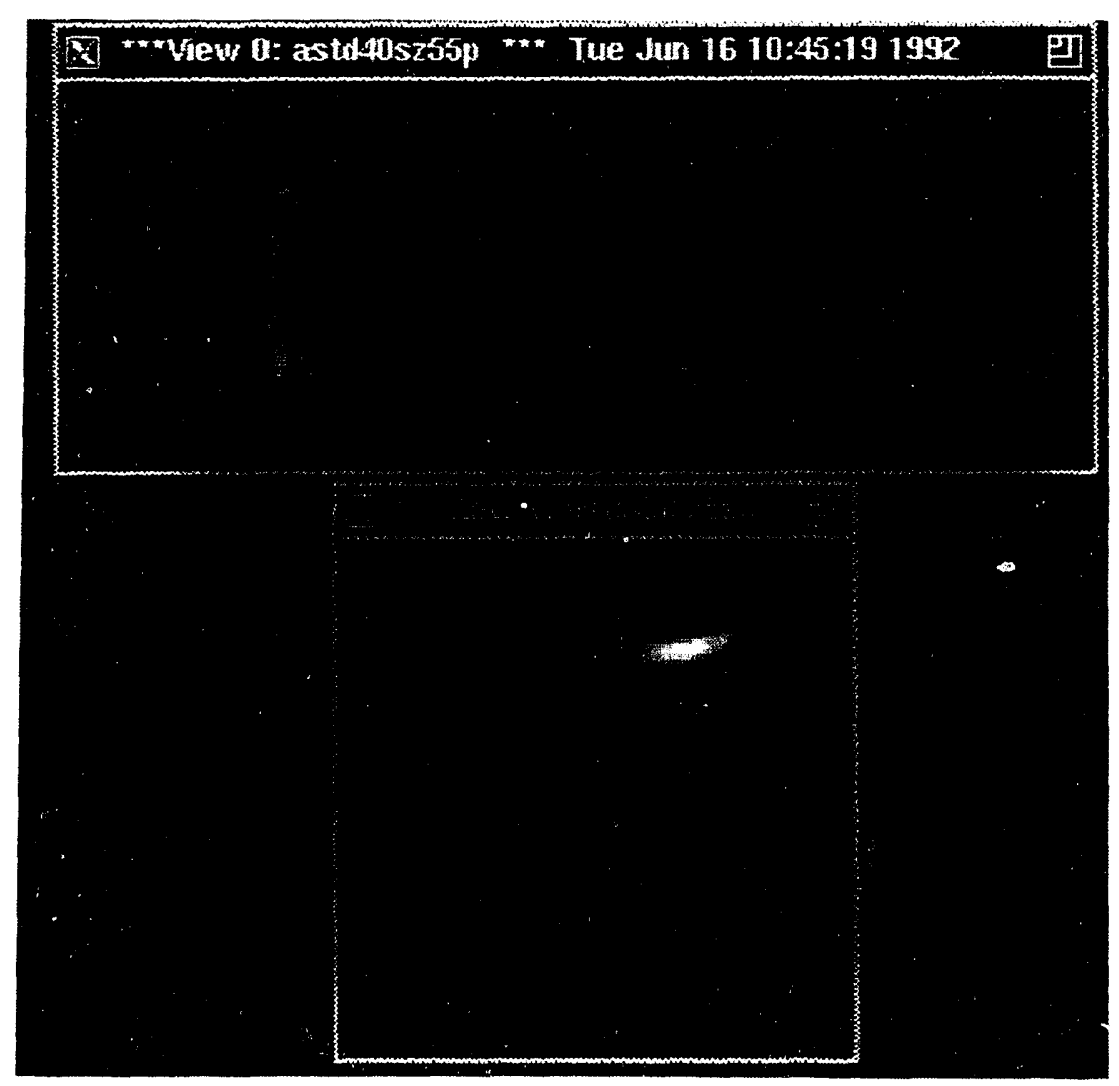

Figure 23b. Image of $55 \mathrm{~mm}$ void post-adaptive-filter weights process. 


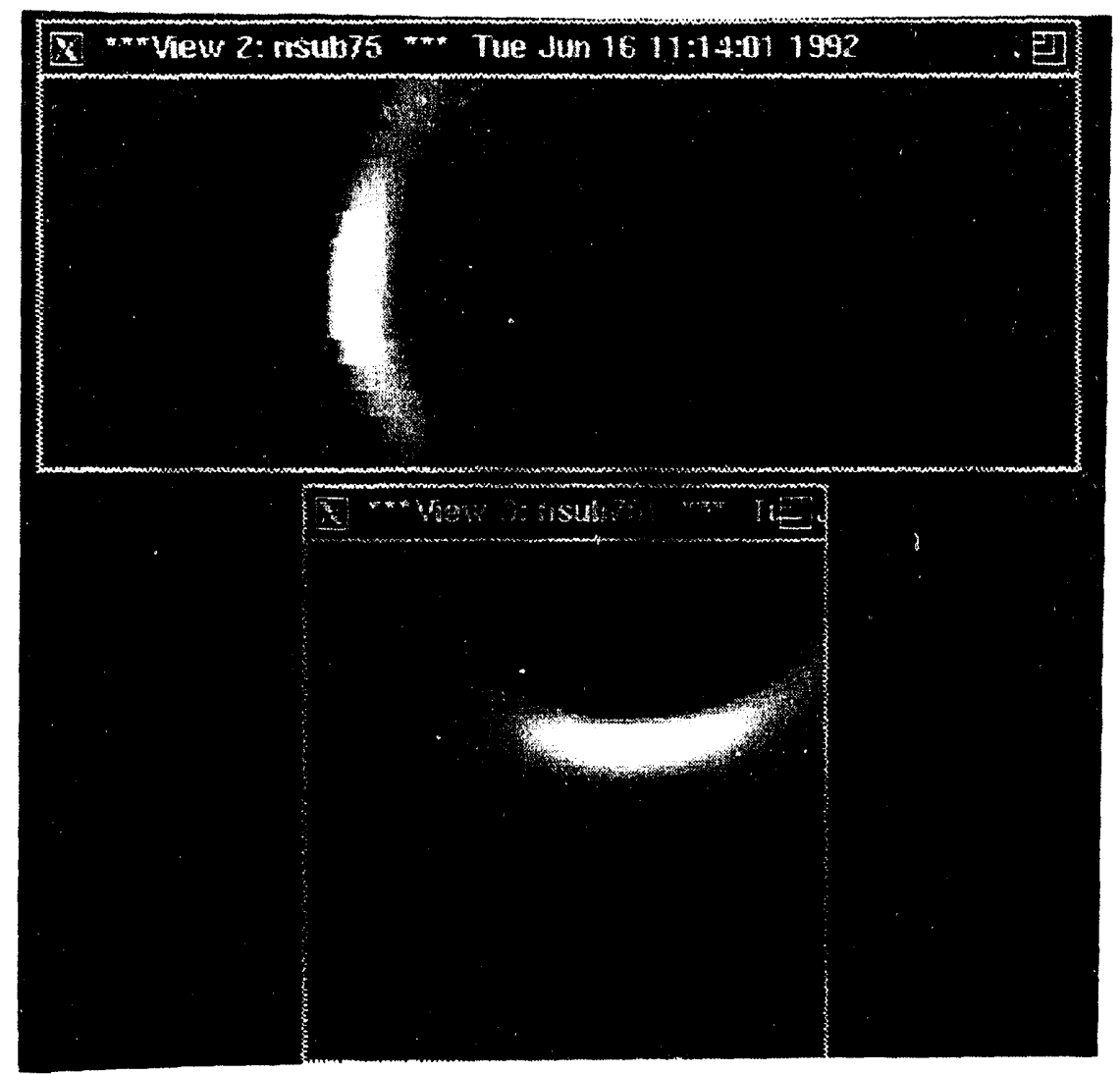

Figure 24a. Image of $75 \mathrm{~mm}$ void before applying adaptive-filter weights.

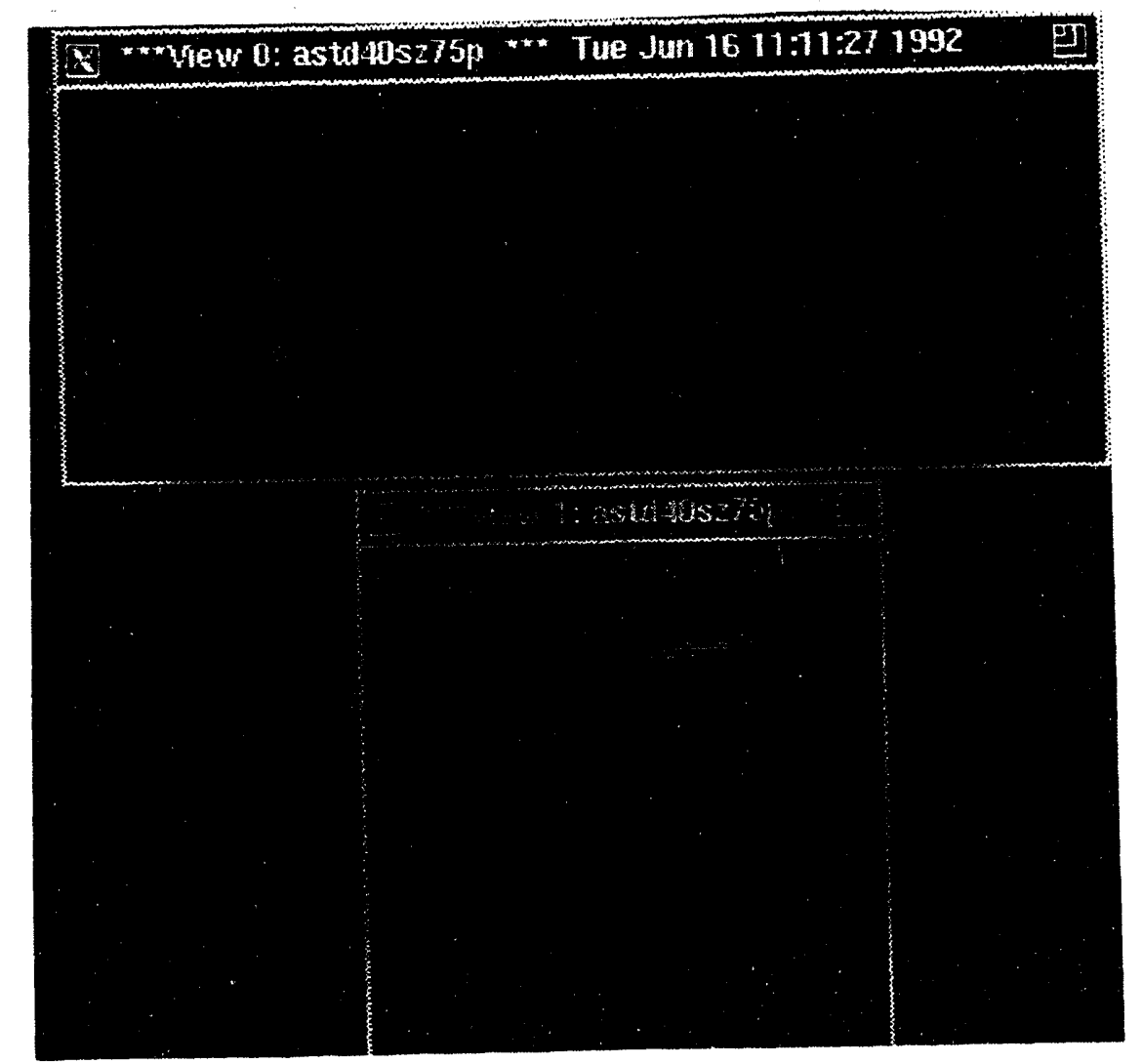

Figure 24b. Image of $75 \mathrm{~mm}$ void post-adaptive-filter weights process. 


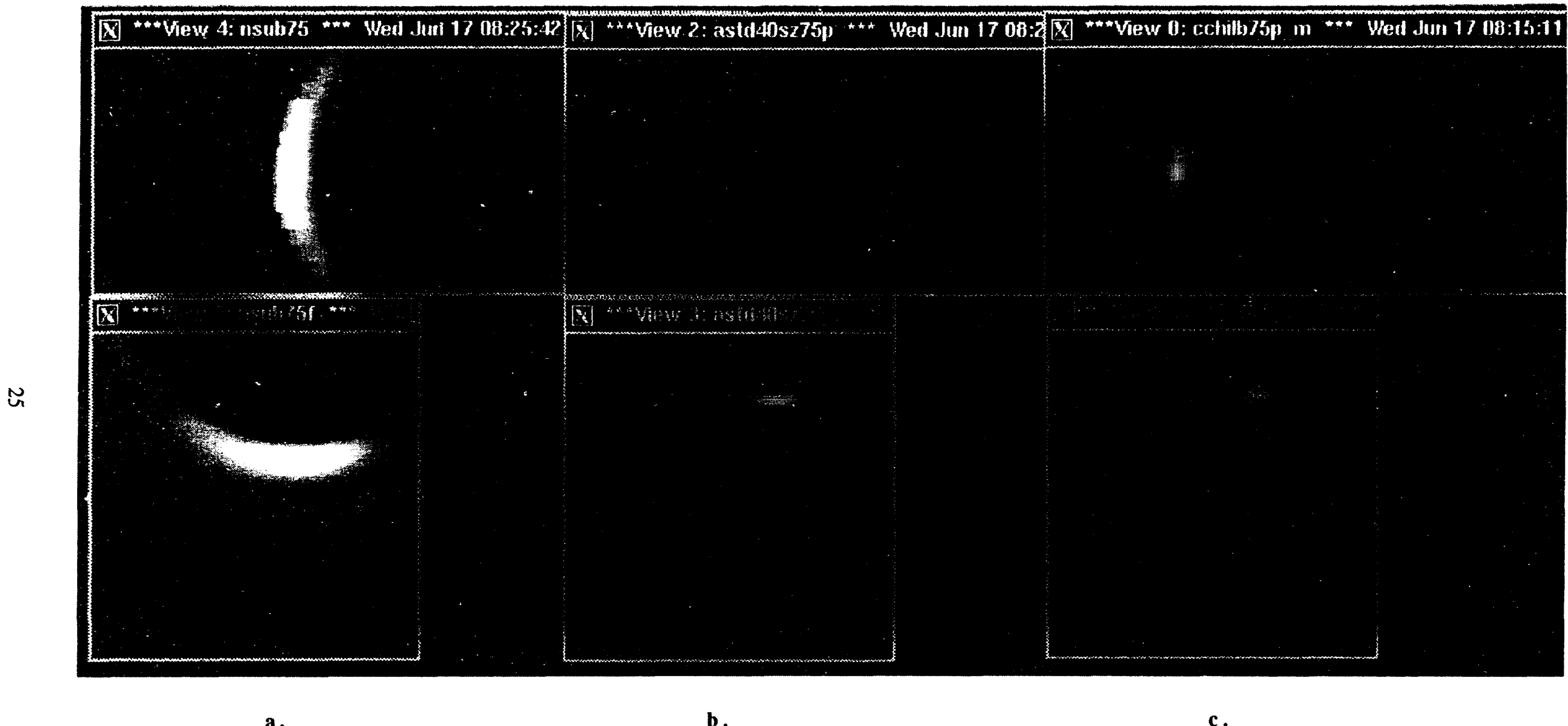

Figure 25: Image of a $75 \mathrm{~mm}$ void:

a. Image of receiver signals without any signal processing;

b. Image after convolving adaptive-filter weights with receiver signals;

c. Image after applying analytic signal to results from the convolution between adaptive-filter weights and receiver signals 


\section{Variable Depth Single Target in Dispersive Concrete}

\subsection{Model-Data Configuration}

The configuration of the single-target model is similar to Figure 1, with 15 receivers and a single transmitter located in the center of the target. The target measured $15 \mathrm{~mm} \times$ $15 \mathrm{~mm}$ and was enclosed in dispersive cement. As with previously discussed models, the aggregate had a dielectric constant of 4.3 , conductivity of $1 e-7$, and individually measured $4 \mathrm{~mm} \times 5 \mathrm{~mm}$. The target was a void filled with dry air and its depth varied from $10 \mathrm{~mm}$ to $150 \mathrm{~mm}$. The transmitted pulse also peaked at $0.4 \mathrm{~ns}$ and 100-ps FWHM.

These data were also supplied with a set of waveforms that contain no target reflection. As discussed in Section 2 and 3, these data were used to remove the coupling pulse by simply subtracting the data set without the void from the different depth void data.

The experiment objective was the development of a set of weights for a given depth and the application of these weights to other data for voids of different depths. In this case, the data for receiver position 1 at a depth of $80 \mathrm{~mm}$ was selected as the waveform to generate the standard set of weights.

\subsection{Adaptive Filter Configuration and Resultant Images}

Figure 8 displays the adaptive filter used to determine the inverse-impulse response for the void in dispersive cement. The operation of the filter is identical to the filter discussed in Section 2.2 .

With the variance of the $x$ matrix equal to
1.474 and with 200 weights, the trace of $R$ can be calculated from equation (3). The trace of the $R$ matrix is given as:

$\operatorname{tr}[R]=294.8$.

With the adaptive time constant $\mu$, set to $5 e-5$, the misadjustment resulted as $1.47 \%$ from equation (2). The time constant for the learning curve is given by equation (5) and calculated as:

$\tau_{\mathrm{MSE}}=3392$ iterations

For five time constants, the ideal total time to adaptation is determined in approximately 17,000 iterations. The adaptive filter performed 80,000 iterations.

An $80-\mathrm{mm}$ void depth was used to determine the standard set of weights. The waveforms for receiver 1 are shown in Figures 26a-c. Figure 26a shows the coupling pulse and the reflection caused by the single void, while Figure $26 \mathrm{~b}$ contains no void reflection. Figure $26 \mathrm{c}$ had the coupling pulse removed by subtracting Figure 26b from Figure 26a. Note that only 300 sample points were provided for these data.

The waveform to obtain the inverseimpulse response for the void at the $80-\mathrm{mm}$ depth only included 200 sample points from Figure 26c, samples 50-249. As in Sections 3 , to save computation time, 200 points were used instead of 300 samples. 


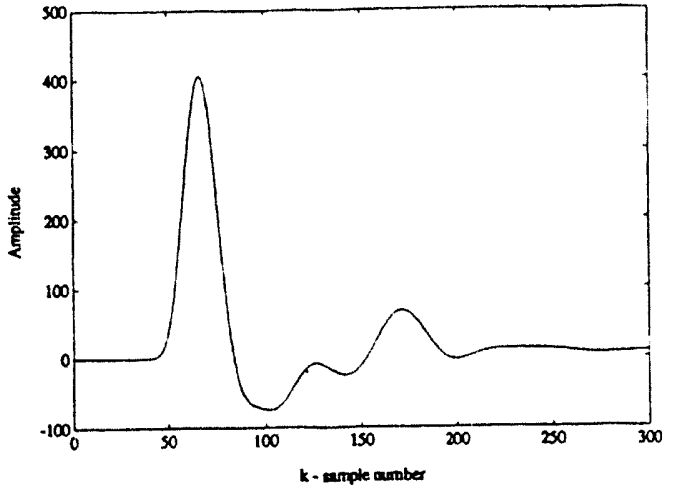

Figure 26a. Single void with coupling pulse.

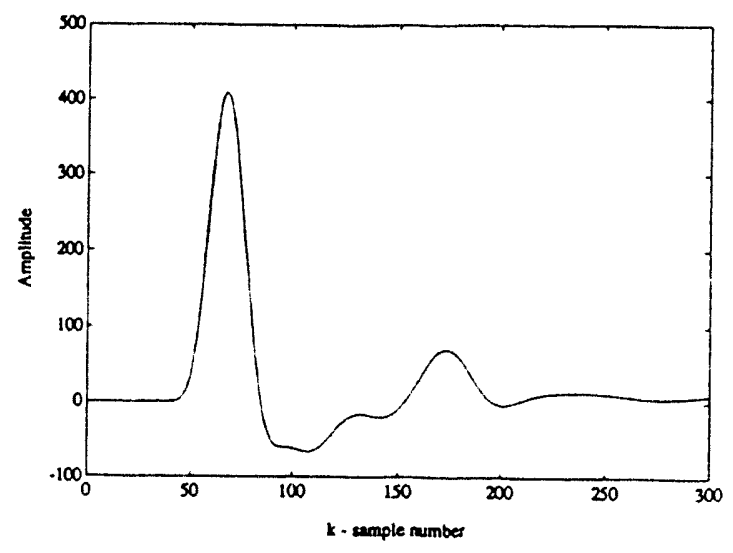

Figure 26b. Received signal without void.

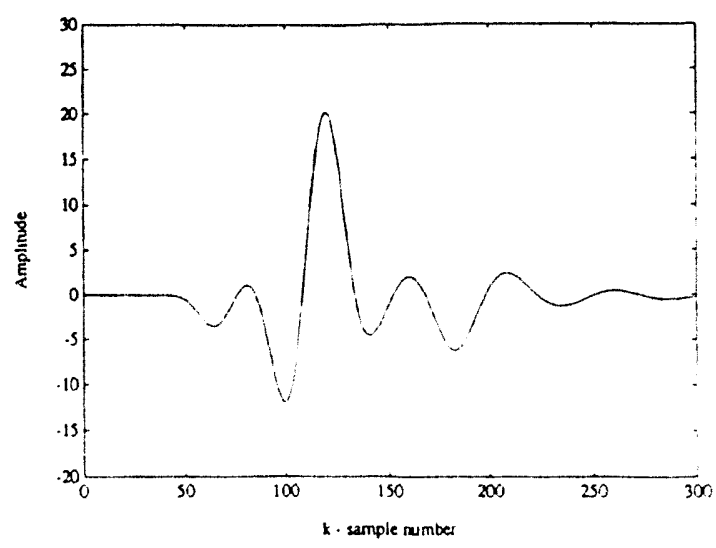

Figure 26c. Void in dispersive medium without coupling pulse.

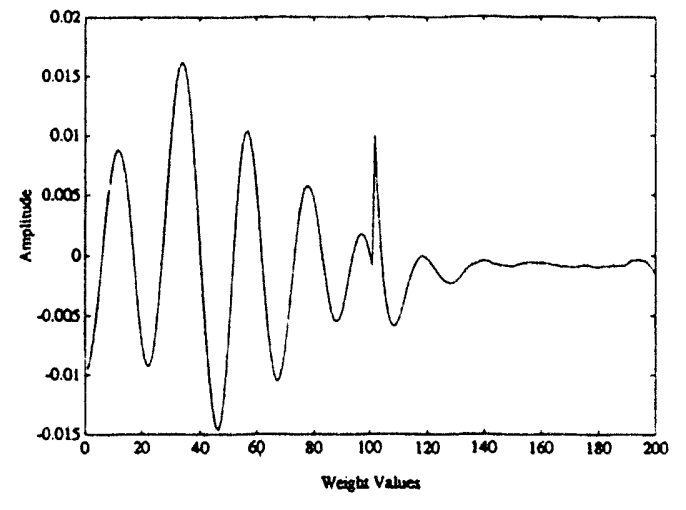

Figure 27. Standard weights for 80 -mm-depth void from receiver 1 .

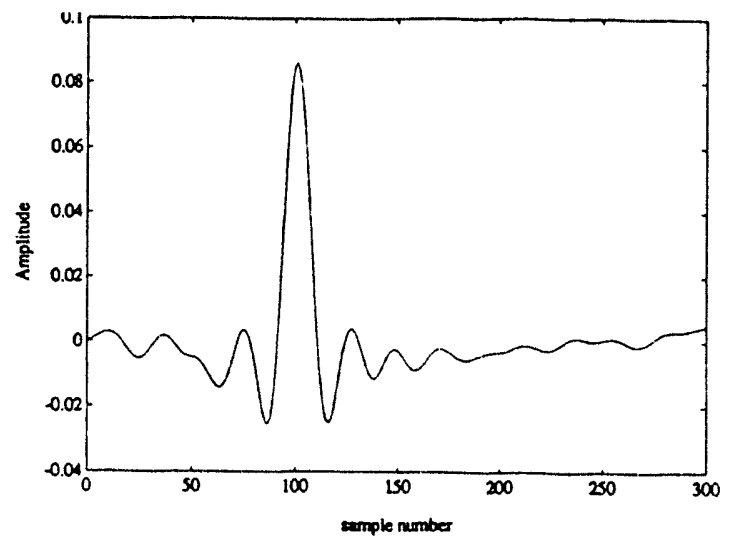

Figure 28. Convolution results of $p$ filter and weights.

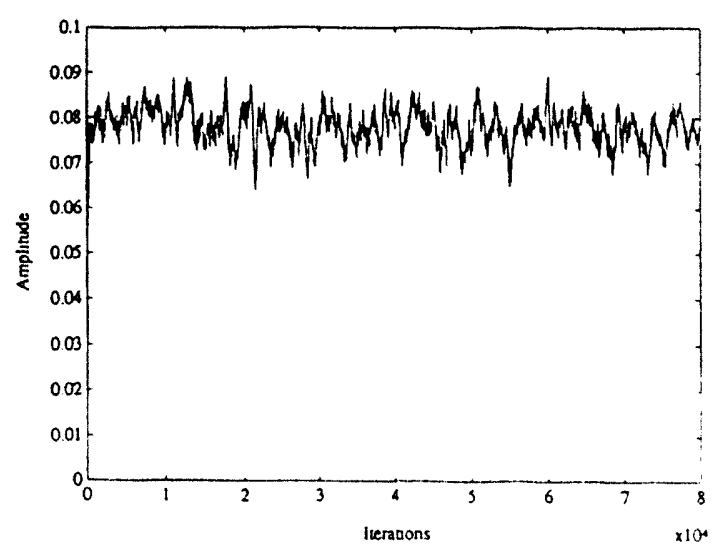

Figure 29. MSE for adaptive filter. 


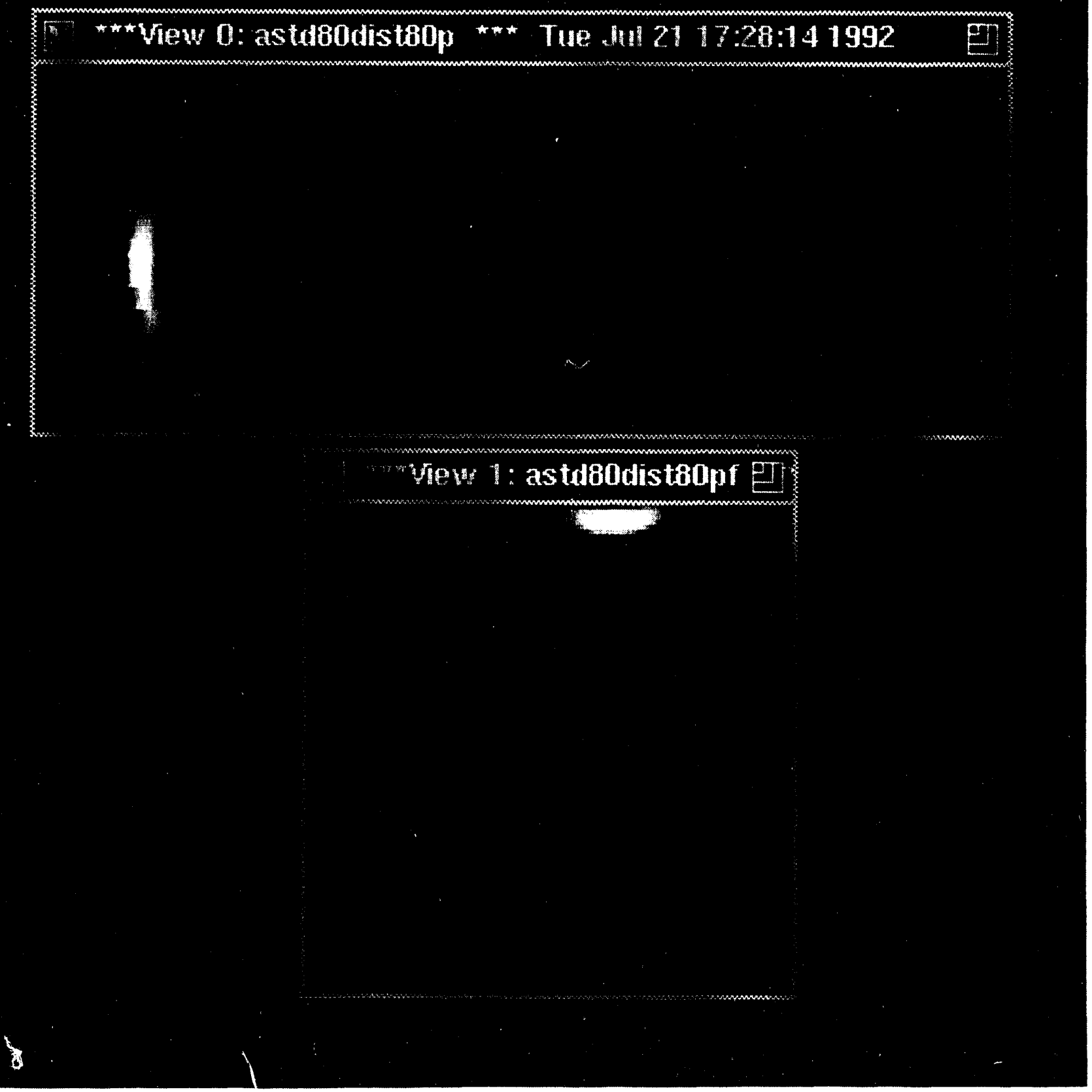

Figure 30. Image of void at a depth of $80 \mathrm{~mm}$.

Figure 27 shows the standard weights determined by the adaptive filter. Figure 28 shows the convolution between the inverse response of the concrete with the contents of the $p$ filter. The resultant waveform, as expected, has an impulsive shape. Figure 29 shows the MSE obtained as before, by averaging the squared error over its 500 nearest neighbors

After the standard set of weights were determined by the adaptive filter for the $80-\mathrm{mm}$ depth void, the weights were applied to the data sets for the $80-\mathrm{mm}, 120-\mathrm{mm}$ and the $150-\mathrm{mm}$ depths. These results are shown in Figures 30, 31 , and 32 , respectively. Because the twodimensional image plane was set to only visualize the targets starting at approximately $80 \mathrm{~mm}$ away from the transmitter, only these images were included in this report. It was determined by using the unfocused timedomain waveforms; however, not imaging 


\section{*triew 0: astd80dist120p *** Wed Jul 22 10:23:36 1992}

,

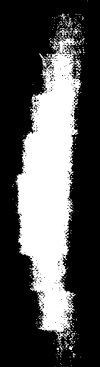

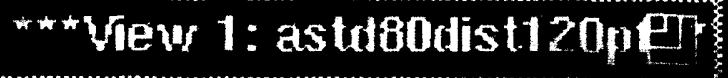

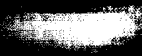

Figure 31. Image of void at a depth of $120 \mathrm{~mm}$.

the data worked equally well. The weights determined from receiver 1 were used on all receiver positions as in Section 3.

Figure 30 shows the image with the least amount of distortion. Because receiver 1 at this void depth was used to generate the standard set of weights, we would expect this image to be the best defined. Figures 31 and 32 show increasing deterioration with an increasing number of artifacts. As the correlation between the $80-\mathrm{mm}$ void data and the other depth signals diminishes due to dispersion, the number of artifacts in the image increases. We could estimate from Figure 32 that the $150-\mathrm{mm}$ void data would be the maximum limit for using the weights from the $80-\mathrm{mm}$ depth. Selecting this range was subjective and was based entirely on the fact that the "cat whiskers" begin to be significant in this range. Even though dispersion has moderately affected the image generated in Figure 32, the location of the void is imaged correctly. 


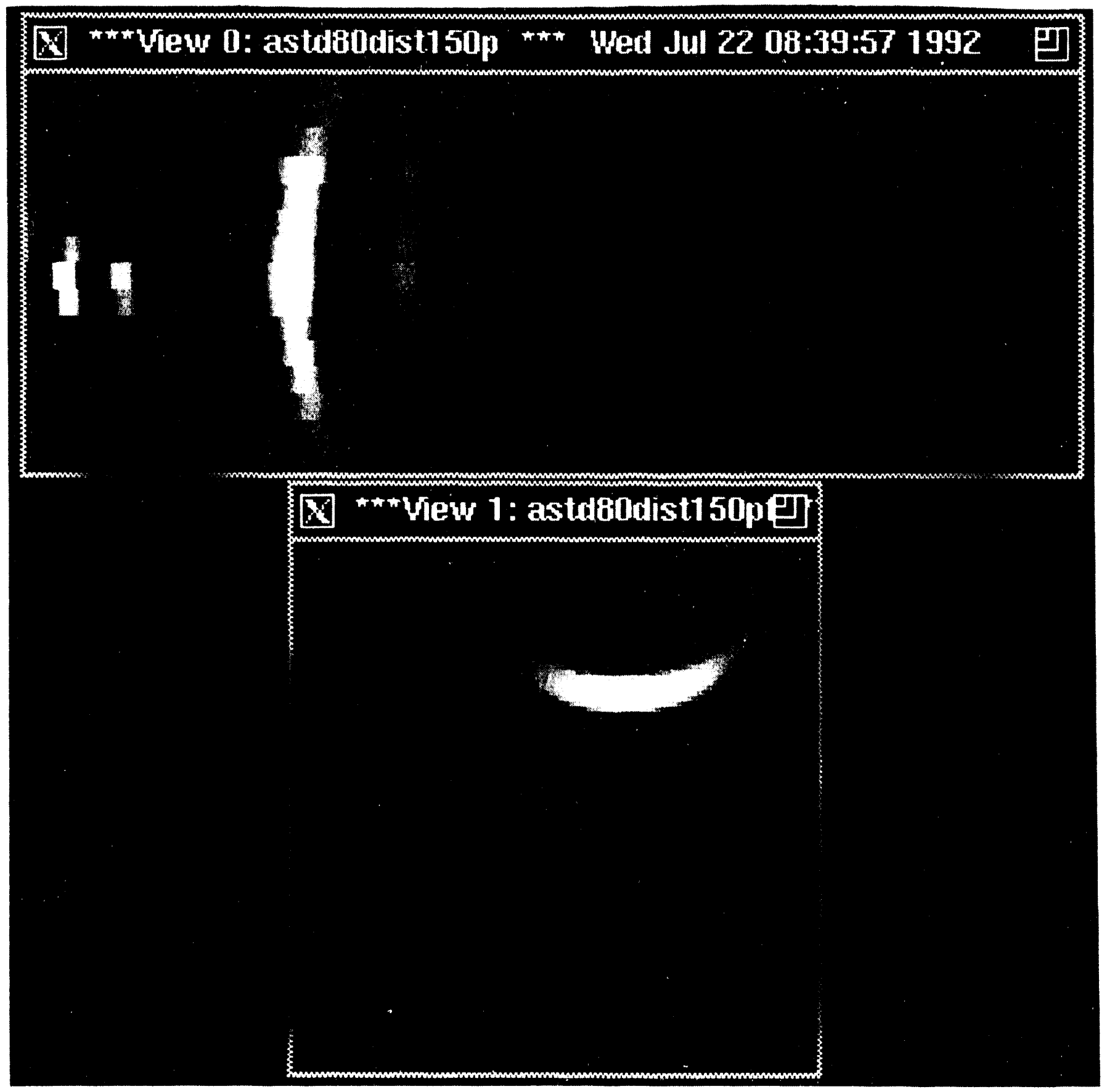

Figure 32. Image of void at a depth of $150 \mathrm{~mm}$. 


\section{Variable Pulse-Width-Transmitted Signal with Single Target in Dispersive Concrete}

\subsection{Model Data Configuration}

The configuration of the model used to generate this data is again similar to Figure 1. The system consists of one transmitter and fifteen receivers centered approximately over the target. The target was in dispersive concrete. The front surface of the rectangularshaped target was located at a depth of $91 \mathrm{~mm}$; its approximate size is $15 \mathrm{~mm} \times 15 \mathrm{~mm}$. In the variable size and depth data, the target was a void. However, for this section the target will be changed to a rebar. The aggregate had a dielectric constant of 4.3 and a conductivity of $1 e-7$.

The coupling pulse was removed in these data sets as in Sections 2, 3, and 4. That is, the data was supplied with a no-target signal reflection for each receiver so the coupling pulse could be subtracted. The pulse-width of the transmitted signal varied from $100-1000$ ps and had a Gaussian pulse shape given by the following equation:

$$
x(t)=A \cdot e^{-\frac{1}{2} \cdot\left[\frac{t-t_{0}}{t_{1}}\right]^{2}}
$$

where $t_{0}$ is the center of the transmitted pulse, $t_{1}$ is the FWHM time, $t$ is time at a sampling rate of $20 \mathrm{ps}$, and $A$ is the maximum amplitude. Each set of variable pulse-width data sets was supplied with 500 data samples.

Because system limitations require the transmitted pulse not be variable, the objective of generating model data of variable pulse widths was to determine the restrictions on the images produced from the wider pulses. Clearly, the wider pulses are easier to generate by a transmitter, but subsequently the wider pulses also limit the resolution of the image. A target that is temporally smaller than the transmitted pulse width will not image smaller than the reflected pulse but will be dependent on the width of the transmitted pulse. The objective of this experiment was to develop a set of weights for three transmitted pulse widths: 100,500 , and $1000 \mathrm{ps}$. A set of images from these three pulse widths were produced using the same size rebar so that the resultant images could be easily compared.

Since it is easier to realize the hardware required to produce the wider transmitted pulse and because this becomes the more attainable system, a second procedure is proposed to address the wider pulse-width issue. In previous sections the assumption was made that the transmitted pulse was impulsive in nature. This assumption can no longer be considered and thus a slightly different process can be applied to generate a set of weights from the adaptive filter. Since the transmitted pulse shape is known, the impulse response of the concrete can be determined for a specific transmitted pulse using the block diagram of Figure 33.

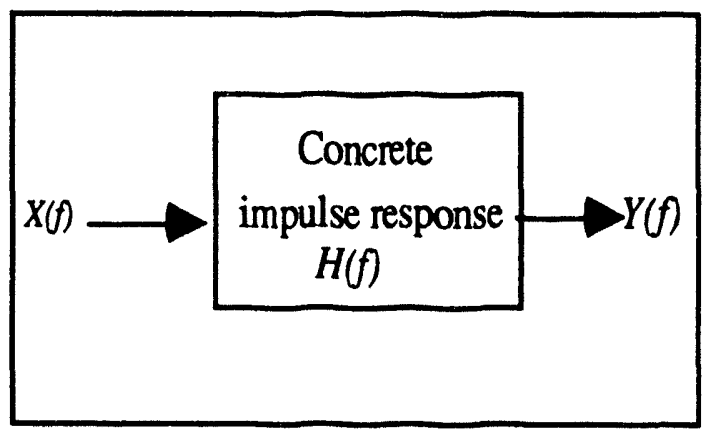

Figure 33. Block diagram of concrete for obtaining impulse response 
The impulse response of the concrete is given by equation (10):

$$
H(f)=\frac{Y(f)}{X(f)}
$$

where $x(f)$ and $y(f)$ are frequency transforms of the transmitted and the received signals respectively. If $h(f)$ is used in the adaptive filter instead of $y(f)$, as was done in the previous sections, the weights will converge to the true inverse-impulse response of the concrete. Thus we can use $h(f)$ or a reduced version of it in the " $p$ " block of the adaptive filter from Figure 8.

After convergence of the adaptive filter, the weights contain the inverse-impulse response of the concrete. This is given as

$$
\hat{\mathrm{H}}^{-1}(\mathbf{f}) \approx \frac{\mathrm{X}(\mathrm{f})}{\mathrm{Y}(\mathrm{f})}
$$

where $\hat{\mathrm{H}}^{-1}(\mathrm{f})$ is the approximate inverseimpulse response of the concrete. Thus, if we consider the frequency transform of the received signals to be $\hat{Y}(f)$, and then divide these signals by the frequency transform of the transmitted signal, the following formulation is determined:

$$
\frac{\hat{Y}(f)}{X(f)} \cdot \hat{H}^{-1}(f)=\frac{\hat{Y}(f)}{X(f)} \cdot \frac{X(f)}{Y(f)}=1
$$

where the assumption that $\hat{Y}(f)$ is approximately equal to $Y(f)$, is made.

Because the inverse frequency transform of 1 is an impulse, equation (12) shows that if the frequency transforms of each of the received signals is divided by the frequency transform of the transmitted signal, the original assumption is valid: the synthetic-aperture focusing technique is imaging from a set of transmitted impulses.

The following section explains the appropriate waveforms obtained by the method just described after the images of the three pulse-widths (100, 500, and $1000 \mathrm{ps)}$ are presented.

\subsection{Adaptive Filter Configuration and Resultant Images}

Figure 8 shows the adaptive filter used to determine the inverse-impulse response for the 100-, 500-, and 1000-ps pulse-widths. Section 2.2 discusses the adaptive filter operation.

The same parameters were maintained in the adaptive filters for the different pulsewidths so that an appropriate comparison could be made. The following parameters apply for all three pulse-widths:

$$
\begin{aligned}
\text { mu } & =.000001, \text { the adaptive time } \\
& \text { constant, } \\
(L+1) & =200, \text { the number of weights, } \\
D & =100, \text { the delay, and } \\
\text { Iter } & =80,000, \text { the number of iterations. }
\end{aligned}
$$

The variance of the $x$ matrix for each of the three different pulse-widths are given as:

$$
\begin{aligned}
\text { variance of } 100 \mathrm{ps} & =8.7466 \\
\text { variance of } 500 \mathrm{ps} & =92.203, \\
\text { variance of } 1000 \mathrm{ps} & =171.57
\end{aligned}
$$

The trace of the $\mathrm{R}$ matrix from equation (3) has the following result:

$$
\begin{aligned}
& \mathrm{tr}[\mathrm{R}] \text { of } 100 \mathrm{ps}=1749.3, \\
& \mathrm{t}[\mathrm{R}] \text { of } 500 \mathrm{ps}=18440.6, \\
& \mathrm{t}[\mathrm{R}] \text { of } 1000 \mathrm{ps}=34314.0 .
\end{aligned}
$$

The misadjustment for each of the pulse-width data set, using equation (2), are:

$$
\begin{aligned}
& \text { Misadjustment of } 100 \mathrm{ps}=.175 \%, \\
& \text { Misadjustment of } 500 \mathrm{ps}=1.84 \%, \\
& \text { Misadjustment of } 1000 \mathrm{ps}=3.43 \%
\end{aligned}
$$



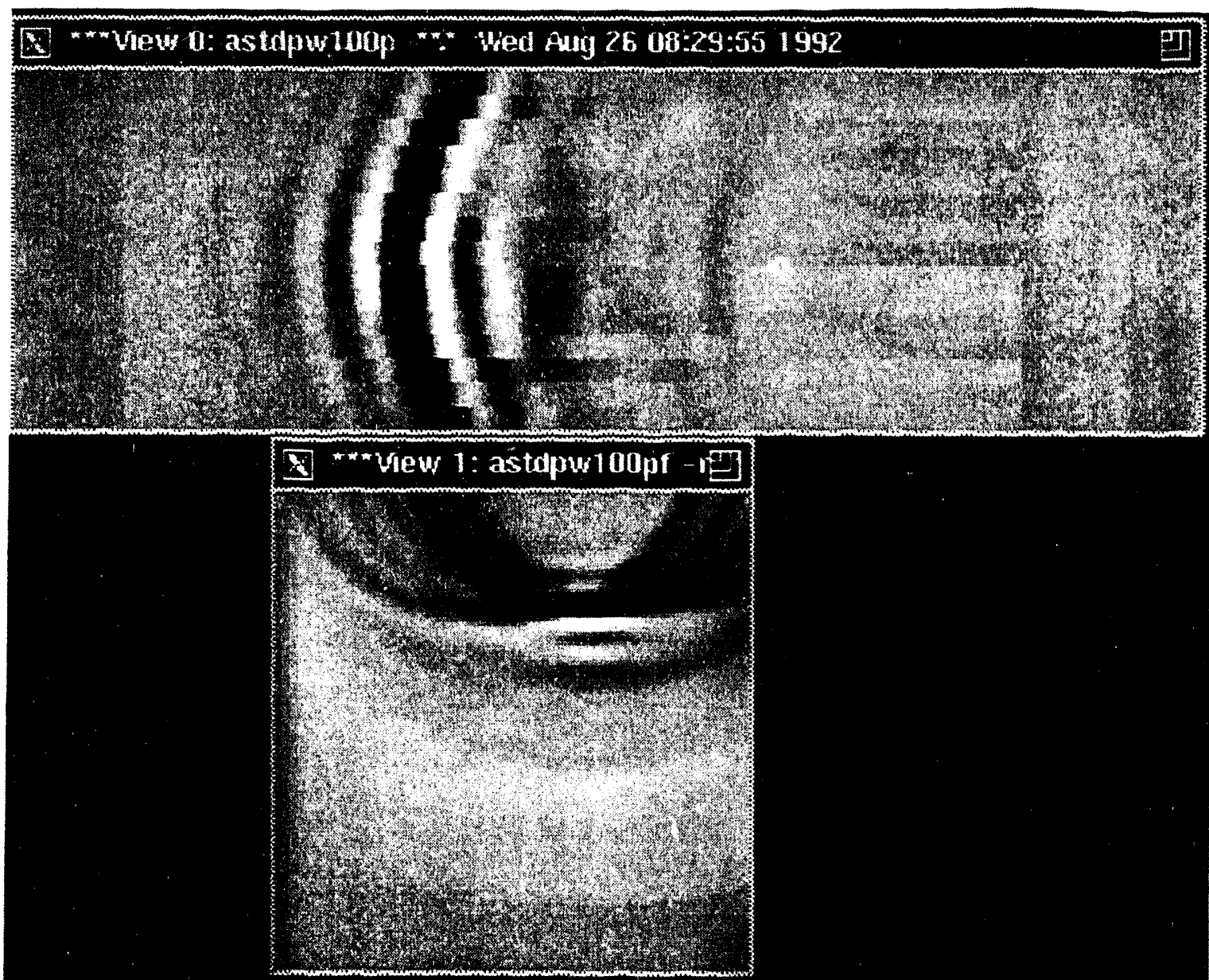

Figure 34. Re-bar image with 100-ps transmitted pulse-width.

The learning curve time constant for the three pulses becomes:

$$
\begin{array}{lll}
\text { for } 100 \mathrm{ps} & \tau_{\text {MSE }}=28583 \text { iterations, } \\
\text { for } 500 \mathrm{ps} & \tau_{\text {MSE }}=2711 \text { iterations, and } \\
\text { for } 1000 \mathrm{ps} & \tau_{\text {MSE }}=1457 \text { iterations. }
\end{array}
$$

After obtaining three different set of weights, one for each transmitted pulse-widths, the image of the rebar was produced by convolving the weights with the other 14 receiver positions. These images are shown in
Figures. 34, 35, and 36 for the 100 , 500- and 1000-ps transmitted pulses, respectively. Once more, the rectangular pictures show the resultant data obtained from the convolution between the weights and the received data before the focusing technique is applied. The square illustration shows the focused picture using the synthetic-aperture focusing technique. The images of Figures. 34, 35, and 36 depend on the transmitted pulse. If obtaining the best resolution is the objective, the 100-ps image is the most desirable.

Since the 1000-ps transmitted pulse image of the rebar is known from Figure 36, a comparison can be made with a second image using the procedure described in the latter 


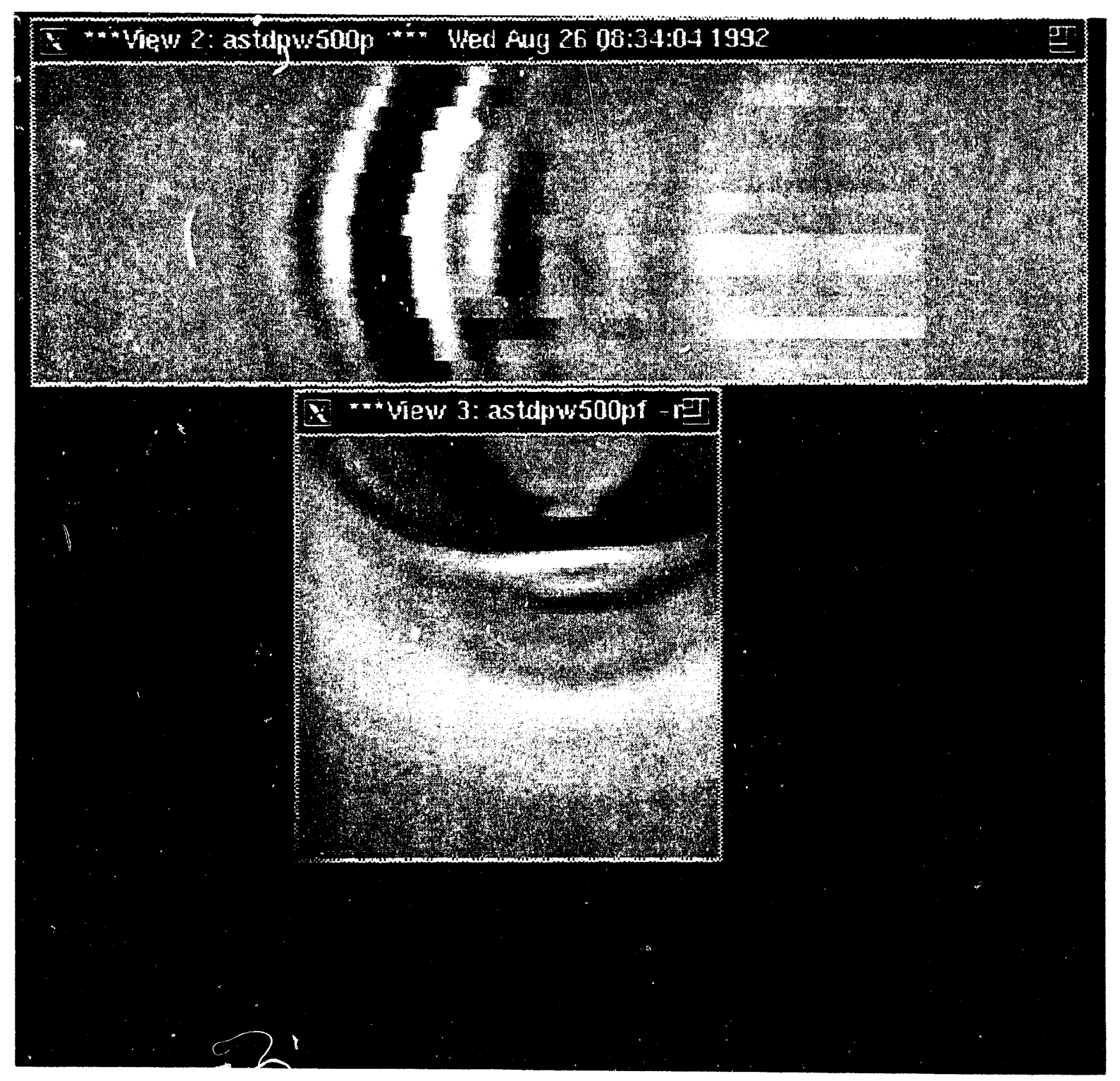

Figure 35. Rebar image. with 500-ps transmitted pulse-width.

portion of Section 5.1.

The parameters selected for the 1000 -ps transmitted pulse-width data using the latter procedure described in Section 5.1 are:

$$
\begin{aligned}
\mu & =.0001 \\
(L+1) & =200 \\
D & =100 \\
\text { Iter } & =80,000
\end{aligned}
$$

The trace of the matrix $R$ is 57.04 after using equation (3) with the variance of $x$ equal to .2852 and with 200 weights for the adaptive filter. With equation (2) and with the adaptive time constant, $\mu$, set to .0001 , misadjustment becomes $.57 \%$. From these values and equation (5), the time constant of the learning curve yields:

$\tau_{\mathrm{MSE}}=8766$ iterations.

Assuming the filter totally adapts in five time constants, 43,828 iterations would be required to reach convergence. For the adaptive filter used in this experiment, 80,000 iterations were processed.

The 1000-ps pulse-width signal from 


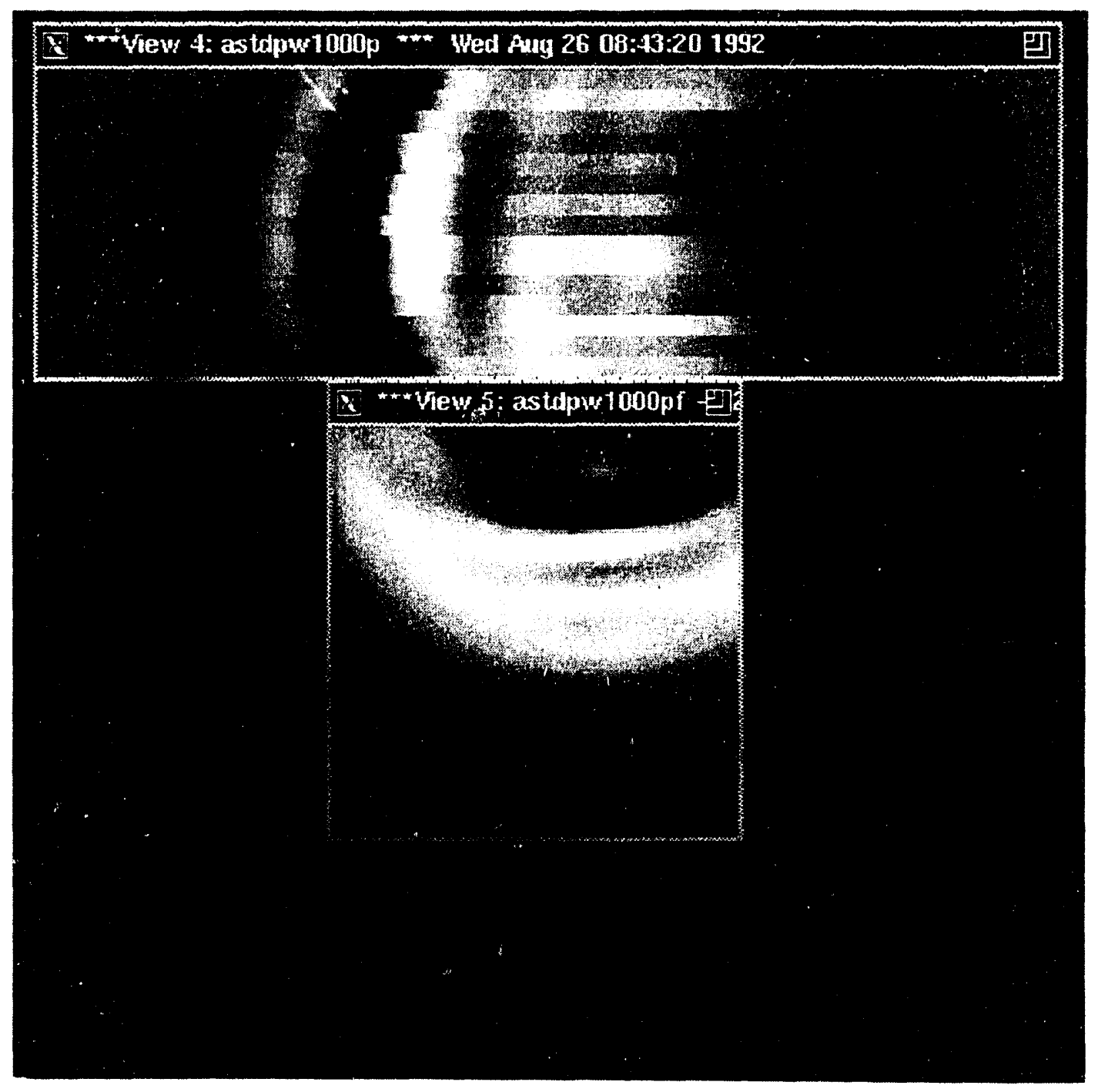

Figure 36. Rebar image with 1000-ps transmitted pulse-width.

receiver position 1 was used to determine the standard set of weights in this experiment.

Figure 37a shows the coupling pulse and the reflection caused by the single rebar; Figure $37 \mathrm{~b}$ displays the received waveform without the target reflection. Note that 500 data samples were supplied for these data sets. Figure 37c represents the difference between Figures 37a and $37 \mathrm{~b}$.

As described in the latter portion of section 5.1, the transmitted pulse and its frequency transform must be determined before the adaptive-filter process is used on variable pulse-width data.
Figure 38 displays the transmitted pulse obtained by equation (9). After acquiring the complex frequency transform sequence of the transmitted pulse and dividing this signal into the complex frequency transform of the received signal, the resultant waveform was converted into the time domain prior to acquiring the contents of the $p$-filter in Figure 8. Figure 39 exhibits the real portion of this time domain signal, which is the impulse response of the concrete. The contents of the $p$ filter is a reduced version of Figure 39, i.e., it contains only samples from 160 to 350 . The contents of the $p$ filter did not consider the imaginary portion of the time-domain sequence signal because, as expected, its amplitude was several orders of magnitude smaller than the real signal. 
Figure 40 shows the weigh's determined by the adaptive filter. Because the transmitted pulse is taken into account, the weights contain the true inverse-impulse response of the concrete. The convolution between the weights and the contents of the $p$-filter is also shown in Figure 41. The resultant waveform is an impulse-shaped signal, as would be expected after convolving the response with its inverseimpulse response. Figure 42 shows the MSE. Figure 43 shows the focused image, after the weights of Figure 40 were determined. Section 2.2 describes the process that produces the images.

Although the size of the target was not changed in this section, the effect of the transmitted pulse is clearly seen in the figures. The significance of considering the transmitted pulse in the adaptive filter process is evident when considering two targets next to each other. If the transmitted pulse is temporally longer than the separation between the two objects, the reflected pulses caused by each target will overlap. However, if the reflected pulses are converted to an impulse-shaped wave form, the target resolution is increased because the target reflections can be closer together befor the equivalent reflected pulses overlay each other. This method of increasing resolution can be a subject of further research.

As an additional illustration of the rebar from the 1000-ps transmitted pulse, an image of the target was generated after optimizing the adaptive filter parameters. Recall that the original reason for selecting the parameters of the adaptive filter were to compare the resultant 1000-ps image with the 100- and 500-ps transmitted pulses. The new optimized values for the adaptive filter and the 1000-ps transmitted pulse are as follows:

$$
\begin{aligned}
\mu & =.0000003 \\
(L+1) & =200 \\
D & =100 \\
\text { Iter } & =50,000 .
\end{aligned}
$$

With these parameters, the variance of the $x$ matrix became 165.695 . The trace of the $R$ matrix is $33,139.0$. The misadjustment is $.994 \%$, and the learning-curve time constant is 5030. Figure 44 shows this new image, along with Figures 36 and 43 for comparison purposes. Figure $44 \mathrm{c}$ show some improvement in the rebar image, even after the adaptive filter parameters were optimized.

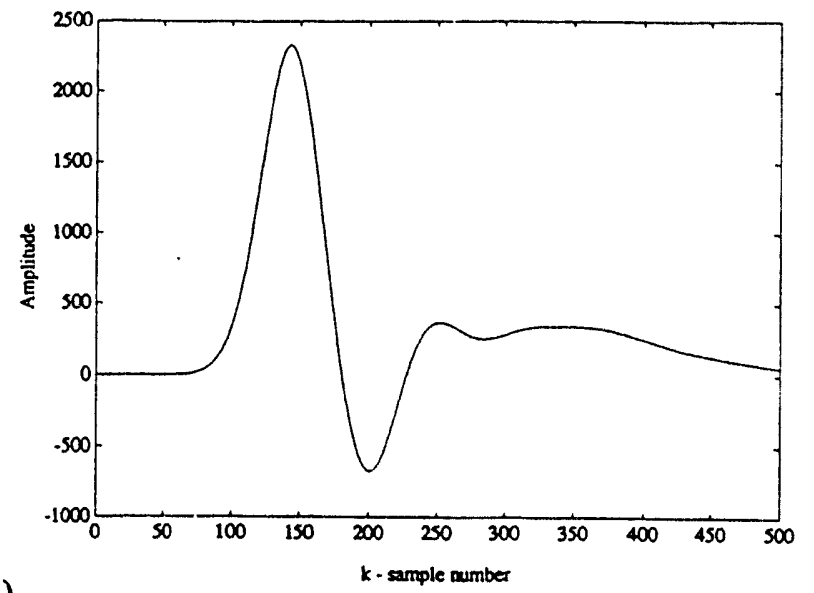

(a)

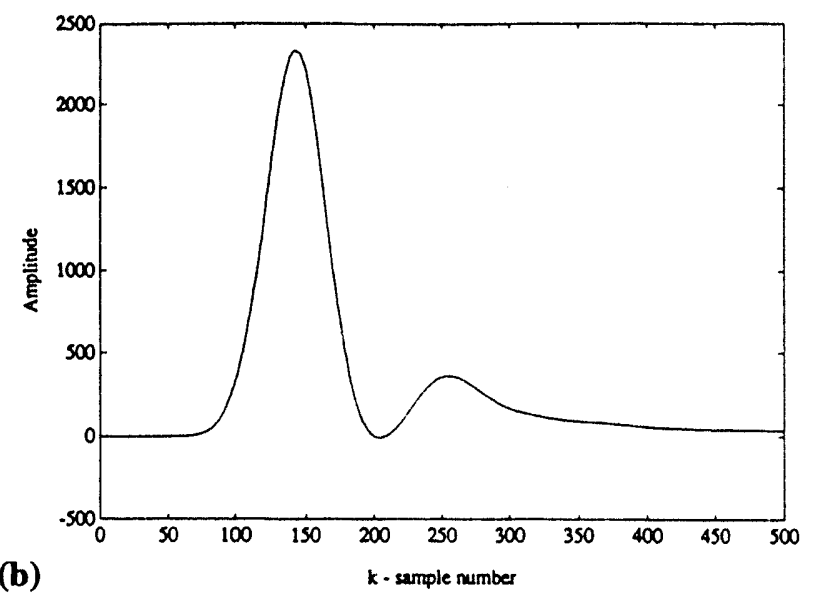

(c)

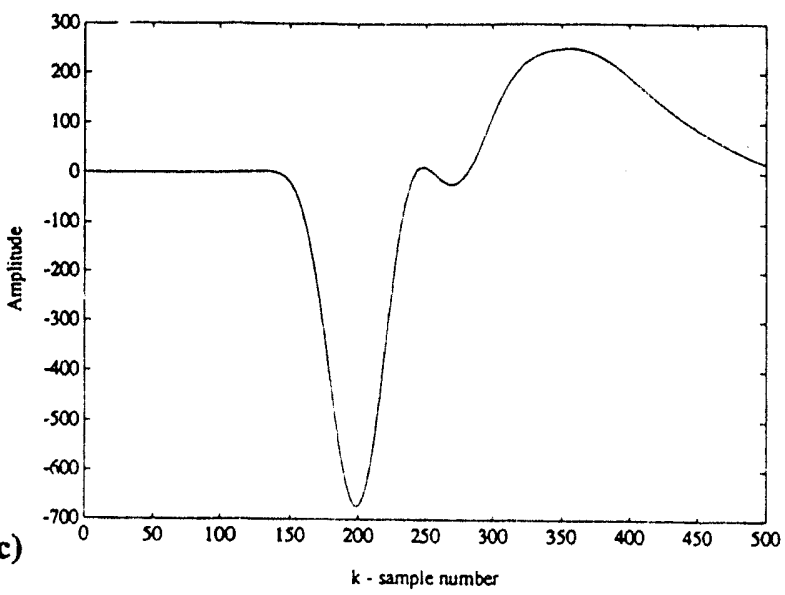

Figure 37. Signals received (a) from rebar in dispersive medium with coupling pulse, (b) without target, and (c) rebar in dispersive medium without coupling pulse. 

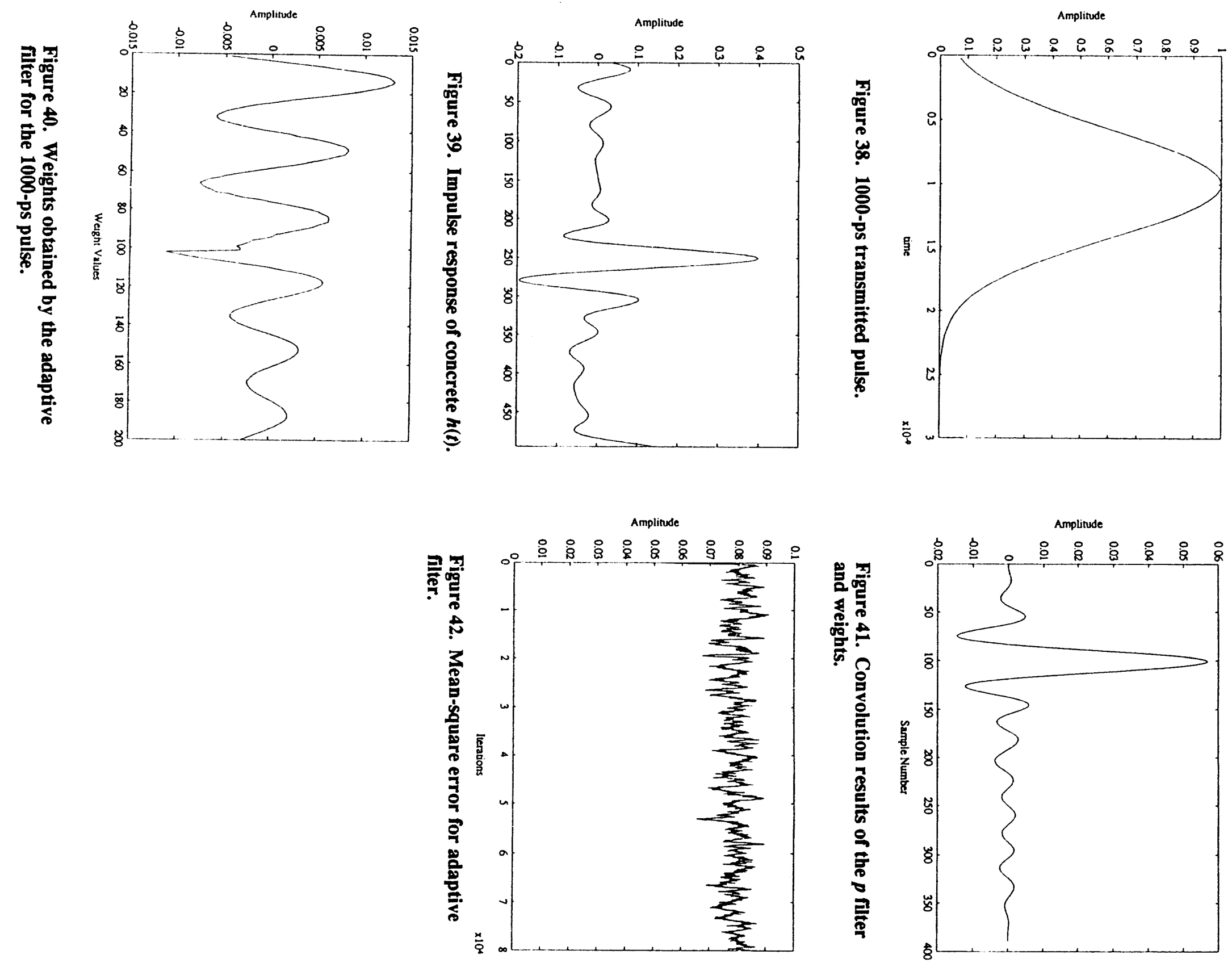

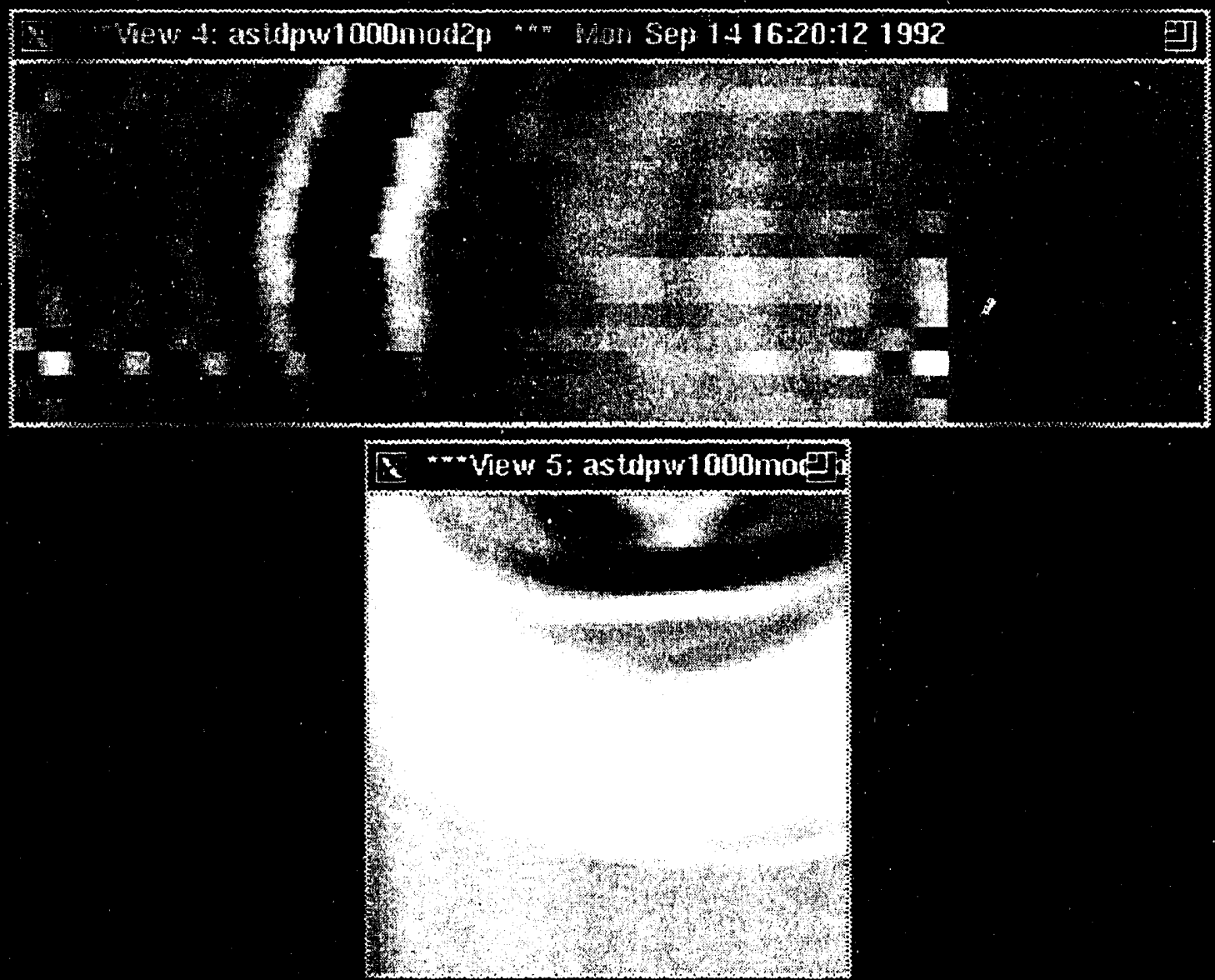

Figure 43. Re-bar image with 1000-ps transmitted pulse using impulse response of the concrete. 


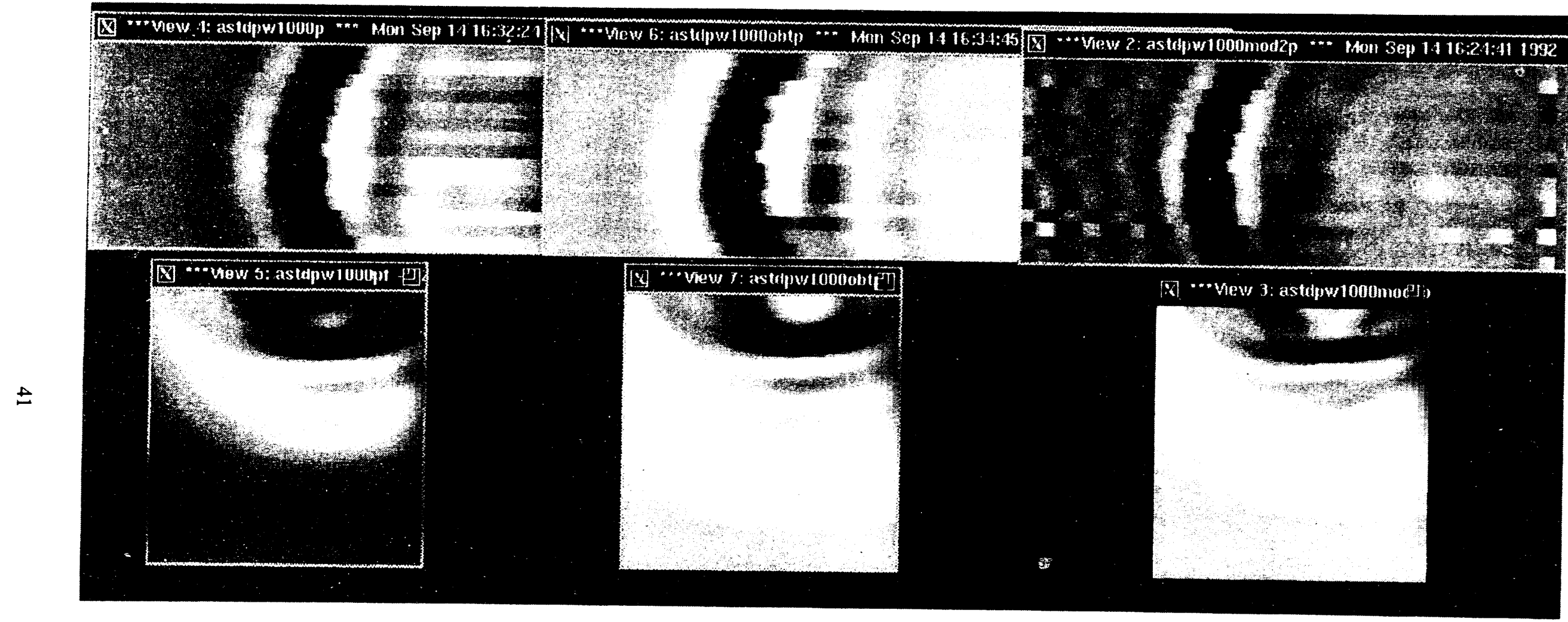

a.

b.

c.

Figure 44 Images of re-bar with $1000 \mathrm{pS}$ transmitted pulse from:

a. First run with adaptive filter parameters using initial values

b. Second run with adaptive filter parameters using optimum values

c. Third run with adaptive filter paremeters using impulse response of concrete 


\section{Time-Domain Synthetic-Aperture Focusing Technique}

The synthetic-aperture focusing technique is a time-domain reconstruction algorithm that enables target identification within an image plane. This focusing technique applies to the Bistatic method of acquiring data, i.e., it is appropriate for one transmitter and multiple receivers model.

The algorithm requires the position of the transmitter and receivers, as well as the dielectric constant of the material in which the target is located. When these information is known by the algorithm, the signals from each of the receivers is spread across an imaging plane. For example, if the target in the concrete generates a signal at sample number 100 for receiver position 1, this datum may have been generated from an equal distance arc away from the receiver. With the receiver at the center, sample 100 is spread evenly across this arc as shown in Figure 45

This procedure is used for each datum sample from receiver 1 and repeated for each receiver until the image in Figure 46 is produced.

Using this method, the signal generated by the target in each receiver will produce an image resulting from the accumulation of the signal at the target location. In the final image plane, the signal constructs an image in the location of the target. This process generates the focused data for this report and explains the reason for the "cat whiskers", i.e., each whisker is the target signal for one of the receivers spread across the image plane. If the number of receivers are increased, the number of artifacts in the images are reduced because each "cat whisker" will have a diminishing effect as the amplitude of the signal at the target location grows larger with each receiver. Clearly, the signal-to noise ratio is increased as the number of receivers is increased.

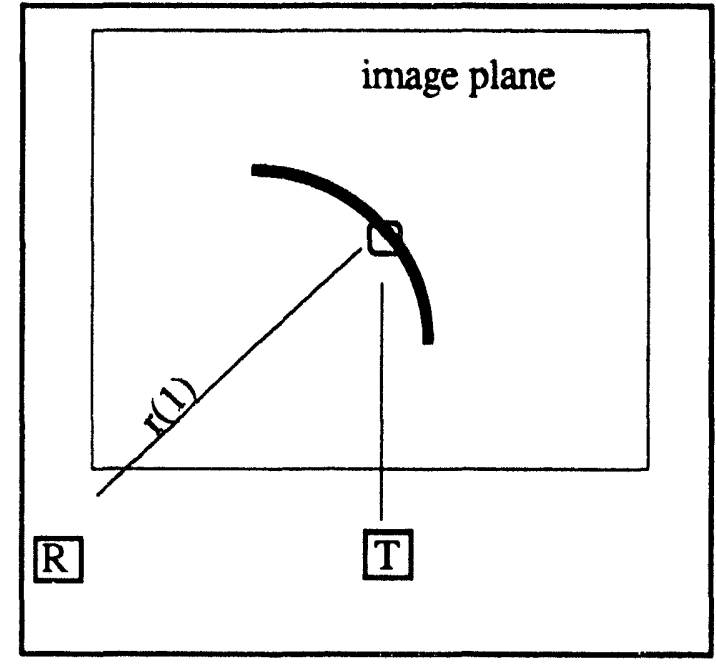

Figure 45. Bistatic focusing technique.

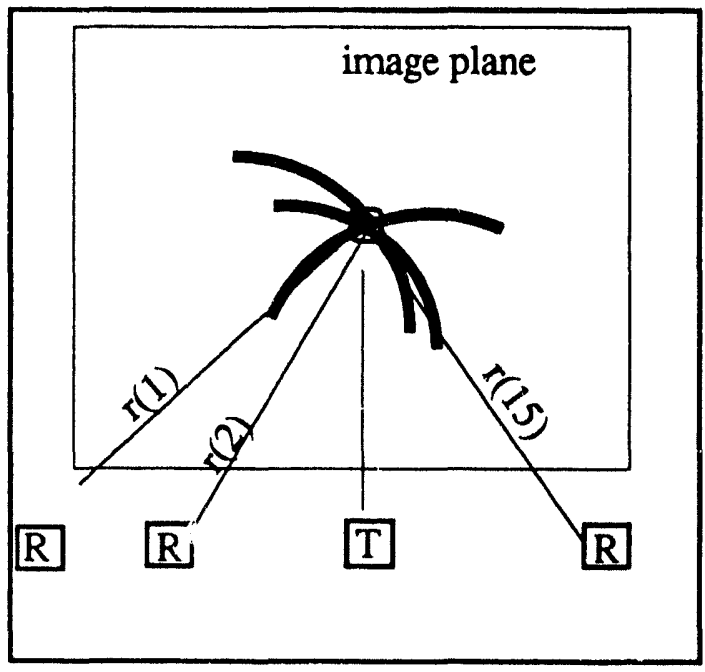

Figure 46. Bistatic focusing technique with multiple receivers. 


\section{Resolution, Sampling and Signal-to-Artifact Ratio}

Several assumptions are necessary to develop first-order approximations for the resolution and the signal-to-artifact ratio of images developed in this repon. These simple assumptions are:

$$
\begin{aligned}
& c= 3 \cdot 10^{8} \mathrm{~m} / \mathrm{sec}, \text { velocity of signal in } \\
& \text { air, }
\end{aligned}
$$

$\mathrm{BW}=2 \mathrm{GHz}$, bandwidth of transmitter,

$r(n)=15$, number of receivers.

The model of Figure 47 shows the diagram of the receivers and the transmitter where:

$$
\begin{aligned}
L & =\text { synthetic aperture width, } \\
T & =\text { location of transmitter, } \\
R & =\text { range, } \\
r(1) \ldots r(n) & =\text { receiver location, } \\
\Delta x & =\text { distance between receivers, } \\
\theta & =\text { transmitted beam width. } \\
v_{p} & =\text { velocity of data acquisition } \\
&
\end{aligned}
$$

With the aid of Figure 47, the following expression can be determined by considering the geometry of the receivers and the transmitter. These are all first-order approximations using a narrow band model

Considering the range of the target and the beam width, half of the width of the synthetic aperture is expressed by:

$$
\frac{L}{2}=R \cdot \tan \left(\frac{q}{2}\right)
$$

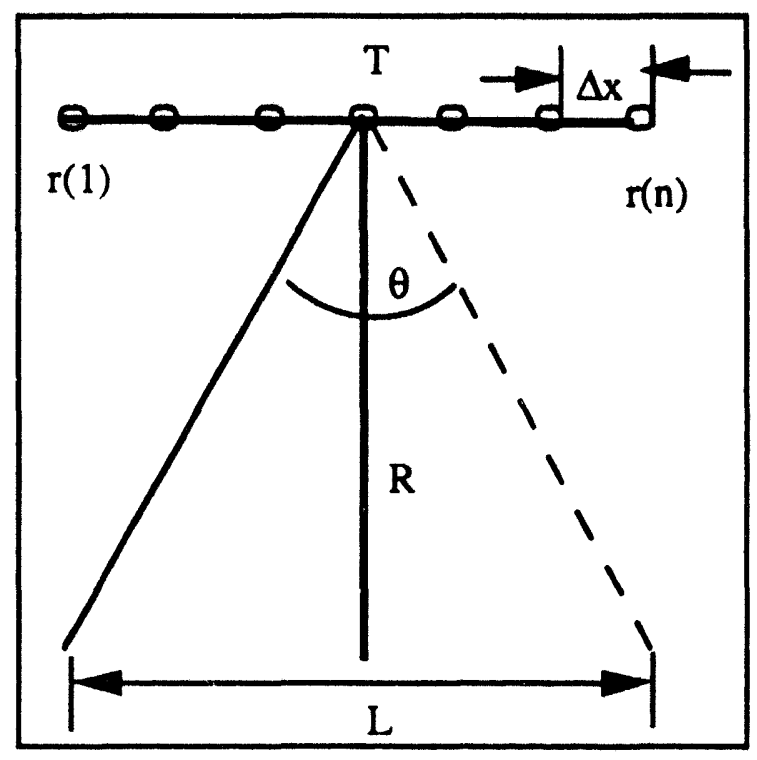

Figure 47. Bistatic model of transmitter and receivers.

To further simplify this expression, we can make the following approximation:

$\tan \left(\frac{\theta}{2}\right) \approx \frac{\theta}{2} \quad$ when $\quad \frac{\theta}{2} \leq 45^{\circ}$

Thus, equation (13) simplifies to:

$L=R \cdot \theta$. 
The cross-range resolution in synthetic apertures is given by:

$$
x_{\text {res }}=\lambda_{c} \frac{R}{L},
$$

where:

$$
\lambda_{c}=\frac{\nu_{c}}{f_{c}}=120 \mathrm{~mm}
$$

$x_{\text {res }}$ is the half-power point of the footprint produced by the synthetic aperture. Equation 16 can be explained intuitively. If the frequency of the transmitter increases, i.e., the wavelength decreases, or if the synthetic aperture increases, the cross-range resolution should increase appropriately, as given by equation (16). Accordingly, if the range increases, the cross-range resolution should decrease.

Using equation (15) in equation (16), with the value determined in equation (17) and $\theta$ equal to $90^{\circ}$, the cross-range resolution becomes:

$x_{\text {res }}=78 \mathrm{~mm}$.

The following equation shows the formulation for the range resolution and it also reveals the resolution's dependence on the bandwidth of the transmitted pulse and the velocity of the wave propagating through the cement:

$$
R_{\text {res }}=\frac{v_{c}}{2 \cdot B W} \text {, }
$$

$$
P R F=\frac{V_{p}}{\Delta x}
$$

where the 2 accounts for the round-trip propagation. Using the values determined previously, the range resolution is given by:

$R_{\text {res }}=30 \mathrm{~mm}$.

If we now consider a system moving along an array of receivers, a synthetic aperture can be created by obtaining samples at a pulse repetition frequency. This pulse repetition frequency is given by :

Because the signal-to-artifact ratio is directly dependent on the number of receivers, the formulation for signal-to-artifact ratio

$\frac{\text { signal }}{\text { artifact }} \approx r(n)=\frac{L}{\Delta x}$,

where $r(n)$ is the total number of receivers.

Using equation (15), with $\theta=1.57$ radians and $\Delta x=15 \mathrm{~mm}$, the resultant equation becomes:

$\frac{\text { signal }}{\text { artifact }}=104.7 \cdot R$,

where the dependence on the range of the target is clearly shown. becomes: 


\section{Summary}

This project demonstrates how a delayinverse-model adaptive filter can be used to improve two-dimensional image formation of targets in a dispersive medium. Once the inverse transfer function is determined by the adaptive filter, the weights can be employed on the other data, in a generic sense. Although a different set of weights were created for each section in this report, this would not be necessary or practical. After determining a standard configuration for a given target, size, and depth, the weights would remain the same. include:

The sets of data evaluated for this report

- Multiple-target data.

- Variable-size target data.

- Variable-depth target data.

- Variable transmitted-pulse-width data.

The inverse-model adaptive filter, in the multiple-target data set, was used to generate a standard set of weights for each receiver from a single target. These weights were applied to the multiple-target data set to generate the image in Figure 14a. The weights generated by the adaptive filter from a void worked equally well with the rebar, i.e., the convolution results produced the proper polarity signal. The final image in Figure $14 \mathrm{c}$ was obtained after several post-adaptive filter processes.

Similarly, a standard set of weights were generated for the variable-size target. Standard weights were produced for a $40 \mathrm{~mm}$ void from receiver position 1 . Recall that the voids varied in size from 5 to $75 \mathrm{~mm}$ in $5 \mathrm{~mm}$ steps. After convolving standard weights with several void sizes, the resulting images were produced in the correct locations. The weights were able to correct appropriately for dispersion in the concrete. These images are shown in Figures $21 \mathrm{~b}$ through $24 \mathrm{~b}$. A post-adaptive-filter (analytic signal) process was used to remove the artifacts created by the limited number of receivers. An image showing the results of the post-adaptive filter technique is presented in Figure 25c.

Another set of weights were generated for the variable-depth target data. These weights were generated from a $80 \mathrm{~mm}$ depth void and applied for the entire depth range (5 to 150 $\mathrm{mm}$ ). Three of the images are shown in Figures 30,31 , and 32; again the weights were capable of compensating the signal dispersion in the concrete. The weights that were generated from the $80 \mathrm{~mm}$ depth data were applicable up to the $150 \mathrm{~mm}$ data, the entire range of the supplied model data. If, however, a target was located at an even lower depth where these standard weights could no longer be used, a second set of standard weights could be generated from a deeper void. Because the reflected signal from the target is dependent on the depth of the target, the standard weights selected to perform the convolution would be dependent upon the time arrival of the received signal.

The inverse-model adaptive filter produced interesting results from the variablepulse-width data. Because the received signal ideally is the transmitted pulse, the rebar image for the 100 to $1000 \mathrm{ps}$ transmitted pulse widths had a significant effect on the image. Figures 34,35 , and 36 showed images for pulse widths of 100,500 , and $1000 \mathrm{ps}$. The image of the target was reasonably improved after considering the transmitted pulse width in the inverse transfer function in the weights generated from the $1000 \mathrm{ps}$ pulse-width data. Again we must mention that any pre-focusing technique can be done "off-line" and therefore the only field operation required is convolving the weights with the receiver data. The image generated using this technique is shown in Figure 43.

The adaptive filter algorithm technique is not the all-inclusive solution for all images generated from ground-penetrating radar. 
However, the we must consider that the adaptive filter has demonstrated it is a very powerful tool that can be used to compensate for dispersion in the transmitting medium. The adaptive filter was capable of generating the appropriate weights regardless of the target type, size, depth, and transmitted pulse width as illustrated in this report. Additionally, other signal-processing tools can successfully uset in conjunction with the adaptive filter methods to improve target images, as was also done in this paper. 


\section{Appendix}

\section{Appendix 9.1 Program Listing of Adaptive Filter Algorithm (written in Matlab for a Mactintosh):}

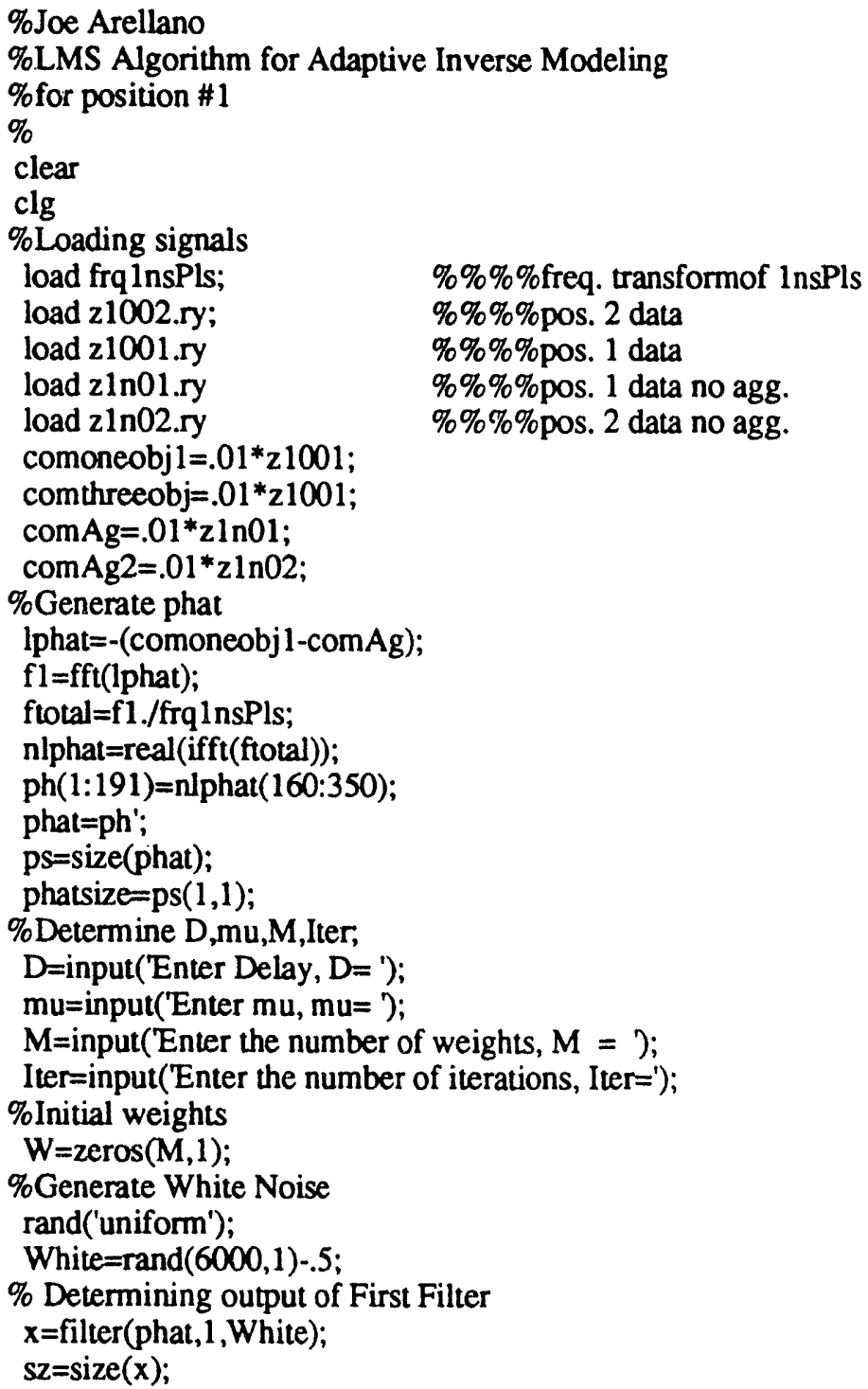

$\% \% \% \%$ freq. transformof 1 nsPls

$\% \% \% \%$ pos. 2 data

$\% \% \% \%$ pos. 1 data

$\% \% \% \%$ pos. 1 data no agg.

$\% \% \% \%$ pos. 2 data no agg. 


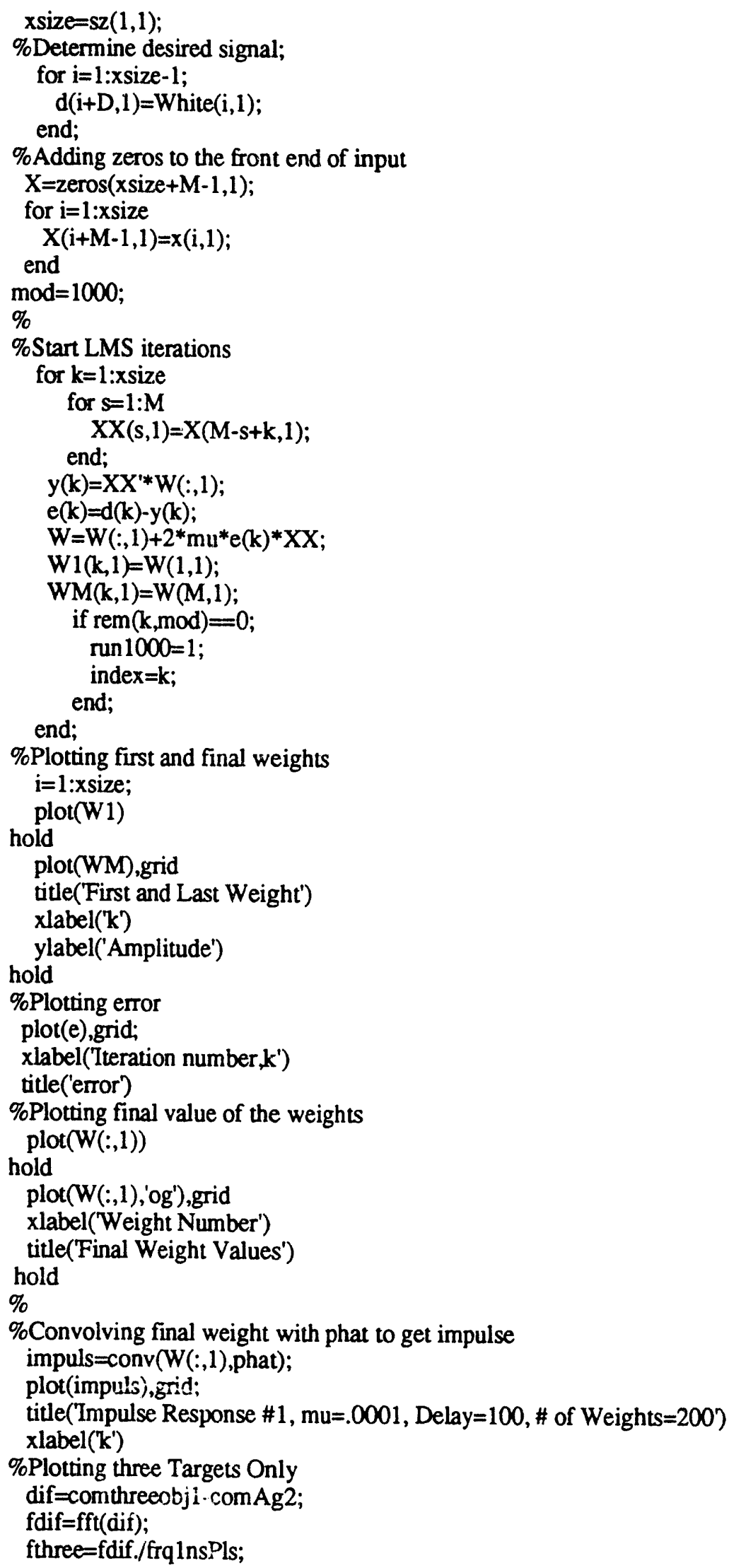




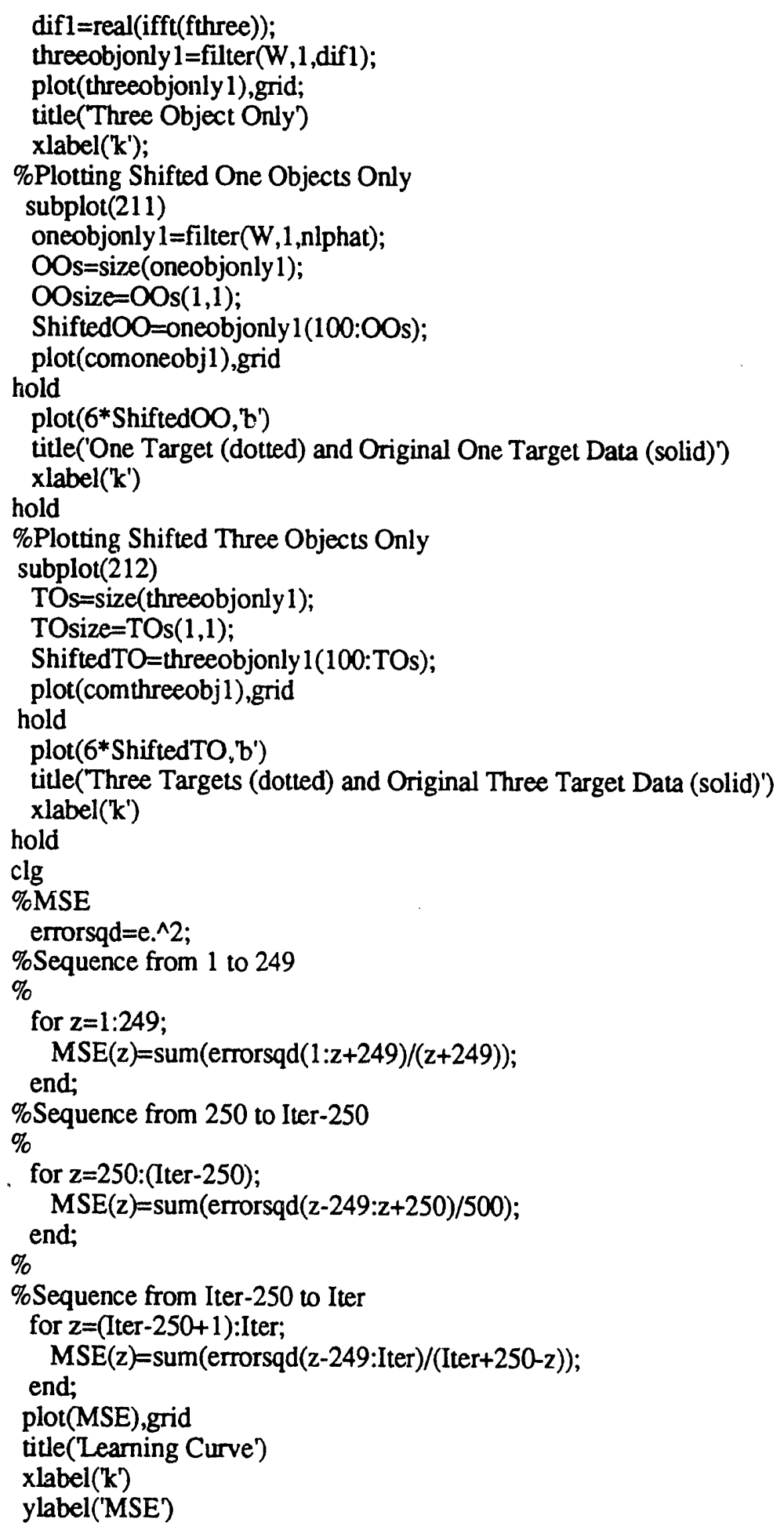




\section{Appendix 9.2 Time-Domain Synthetic Aperture Focusing Technique Program Listing (written in C for Unix)}

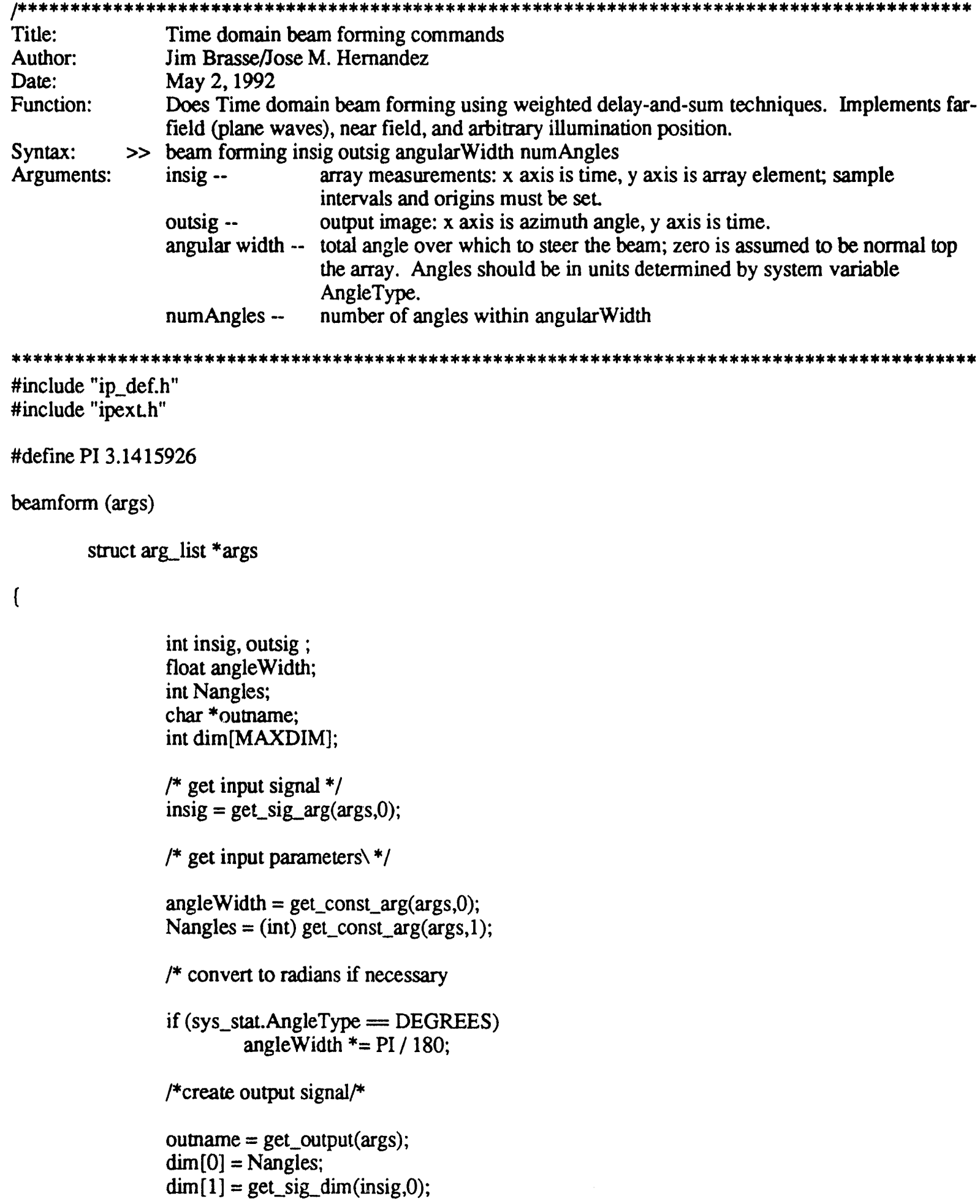




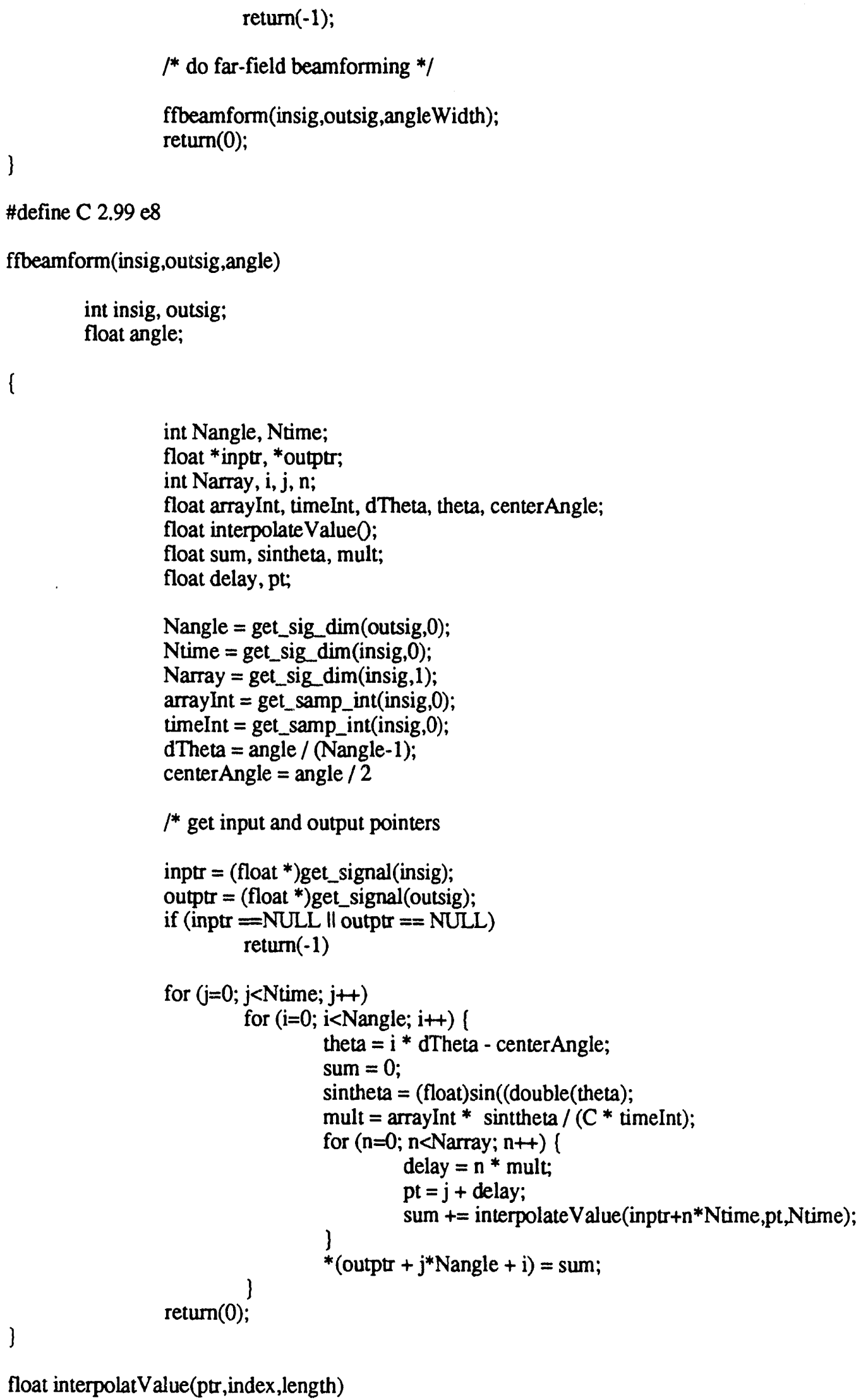

float interpolatValue(ptr,index, length) 
float *ptr, index;

int length;

l

int $\mathrm{m}$;

$\mathrm{m}=$ (int)rint(index);

if ( $\mathrm{m}<0 \| \mathrm{m}>=$ lenth) return $(0)$;

else return $\left({ }^{*}(p t r+m)\right)$;

\}

arrayfocus(args)

struct arg_list *args;

l

int insig, outsig;

float $\mathrm{dx}$, xinit;

int $\mathrm{Nx}$;

char *outname;

int $\operatorname{dim}$ [MAXDIM];

int $\mathrm{Nz}$;

float zinit, dz, xs, zs, dinit;

float de;

$/ *$ get input signal */

insig = get_sig_arg $(\operatorname{args}, 0)$;

$/ *$ get input signal */

insig = get_sig_arg $(\operatorname{args}, 0)$;

/* get input parameters */

$\mathrm{Nx}=$ (int)get_const_arg(args,0)

$\mathrm{dx}=$ get_const_arg(args,1)

xinit $=$ get_const_arg(args,2)

$\mathrm{Nz}=$ (int)get_const_arg(args,3)

$\mathrm{dz}=$ get_const_arg(args,4)

zinit = get_const_arg(args,5)

xs = get_const_arg(args,6)

zs = get_const_arg(args,7)

dinit $=$ get_const_arg(args, 8$)$

de $=$ get_const_arg(args,9)

/* create output signal */

output $=$ get_output(args);

$\operatorname{dim}[0]=\sqrt{ } \mathrm{x}$;

$\operatorname{dim}[1]=\mathrm{Nz}$;

if $(($ outsig $=$ create_sig $($ outname,REAL,2,dim $))<0)$

return(-1); 


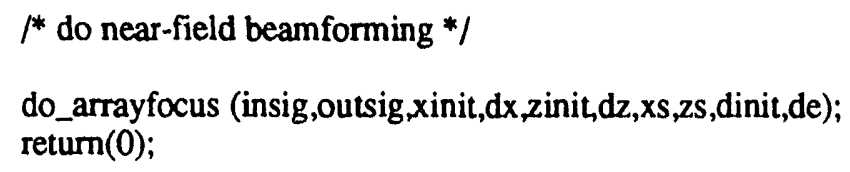

init insig, outsig;

float xinit, dx, zinit, dz, xs, zs, dinit,de;

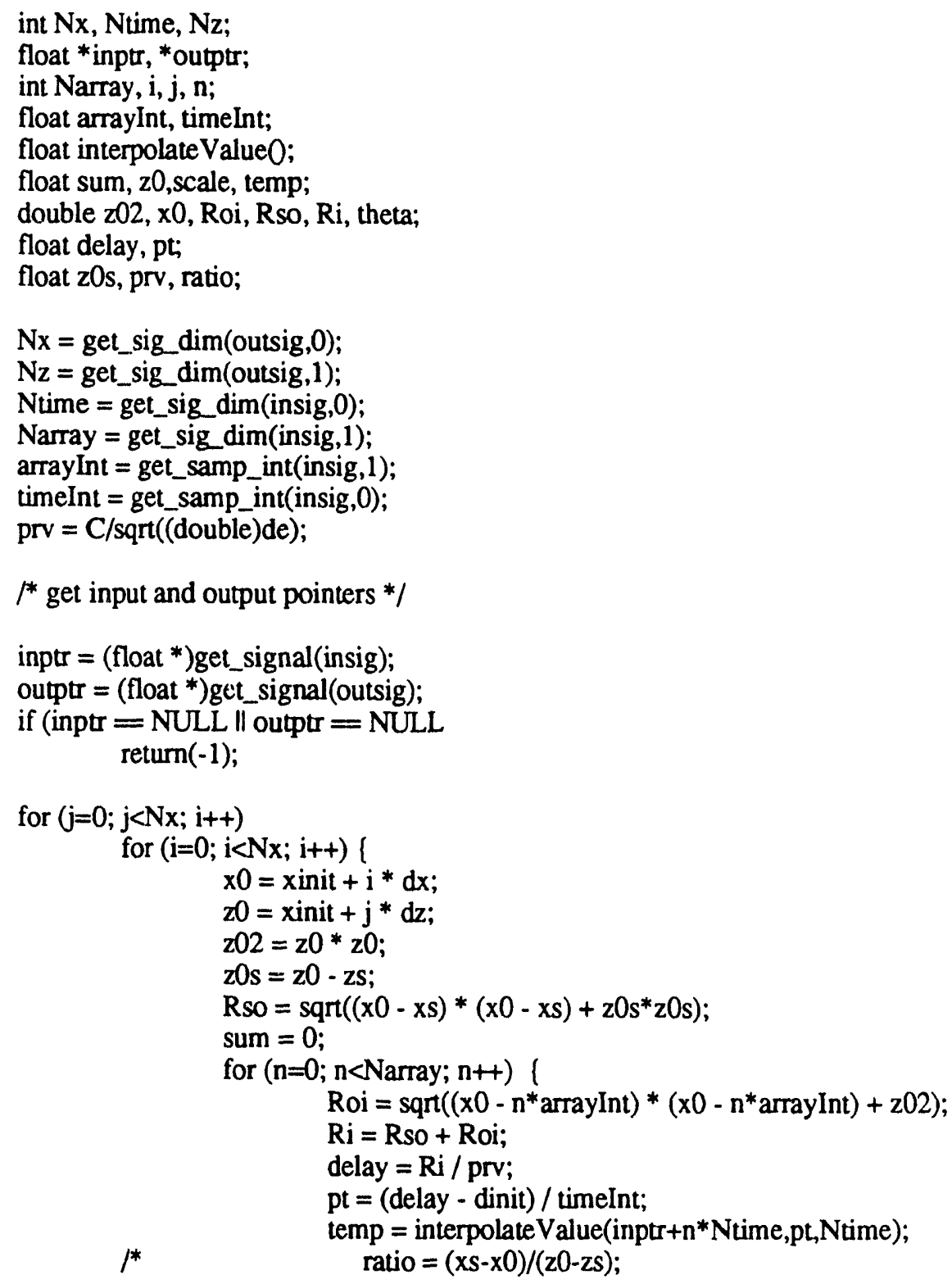




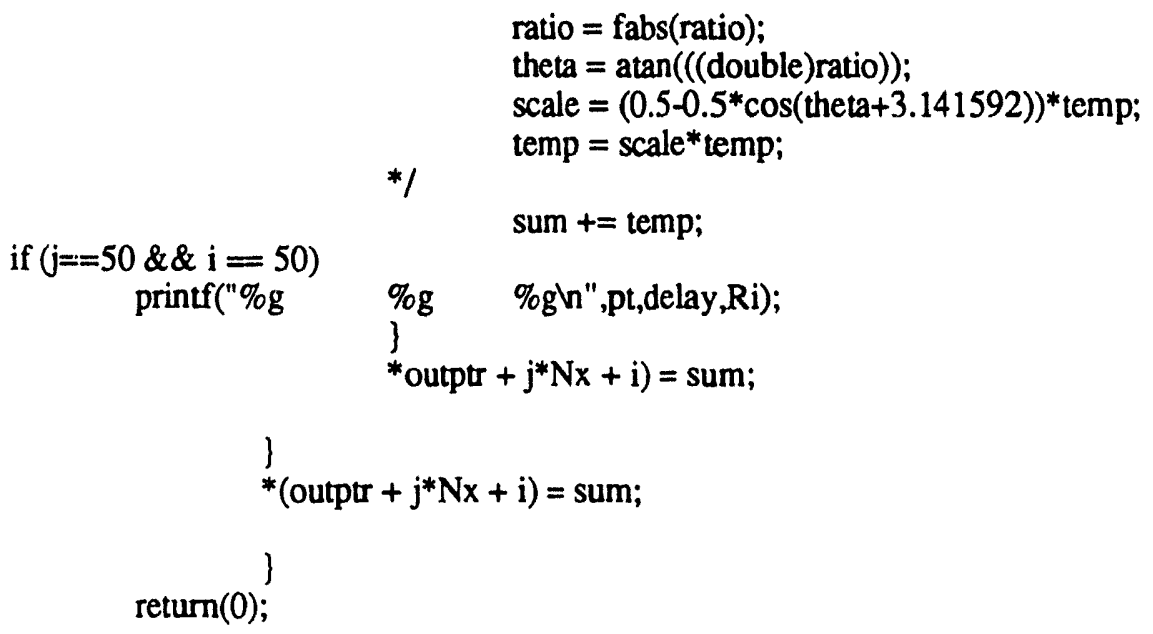


1. H. S. Limaye and R. J. Krause, "Nondestructive Evaluation of Concrete with Impact-Echo and PulseVelocity Techniques," Materials Evaluation, (0025-5327/91 The American Society for Nondestructive Testing, Inc., October 1991).

2. R. Zoughi, G. L. Cone, and P. S. Nowak, "Microwave Nondestructive Detection of Rebars in Concrete Slabs," Materials Evaluation, (0025-5327/91 The American Society for Nondestructive Testing, Inc., November 1991).

3. J. P. Fitch, Synthetic Aperture Radar (Springer-Verlag, New York, NY, 1988).

4. B. Widrow and S. Stems, Adaptive Signal Processing (Prentice Hall, Englewoods Cliff, NJ, 1985).

5. J. Komo, Random Signals Analysis in Engineering Systems (Academic Press, Inc., Clemson, SC, 1987).

6. R. Bracewell, The Fourier Transform and Its Applications (McGraw Hill Book Company, San Francisco, CA, 1986).

7. A. Oppenheim and R. Schafer, Discrete-Time Signal Processing (Prentice Hall, Englewoods Cliff, NJ, 1989).

8. J. Candy, Signal Processing, The Modern Approach (McGraw Hill Book Company, Clemson, SC, 1988).

9. R. D. Strum and D. E. Kirk, First Principles of Discrete Systems and Digital Signal Processing (Addison-Wesley Publishing Company, Menlo Park, CA, 1989).

10. P. M. Gammel, Improved Ultrasonic Detection Using the Analytic Signal Magnitude (Utrasonics, March 1981).

11. K. R. Castleman, Digital Image Processing (Prentice Hall, , Englewoods Cliff, NJ, 1979). 

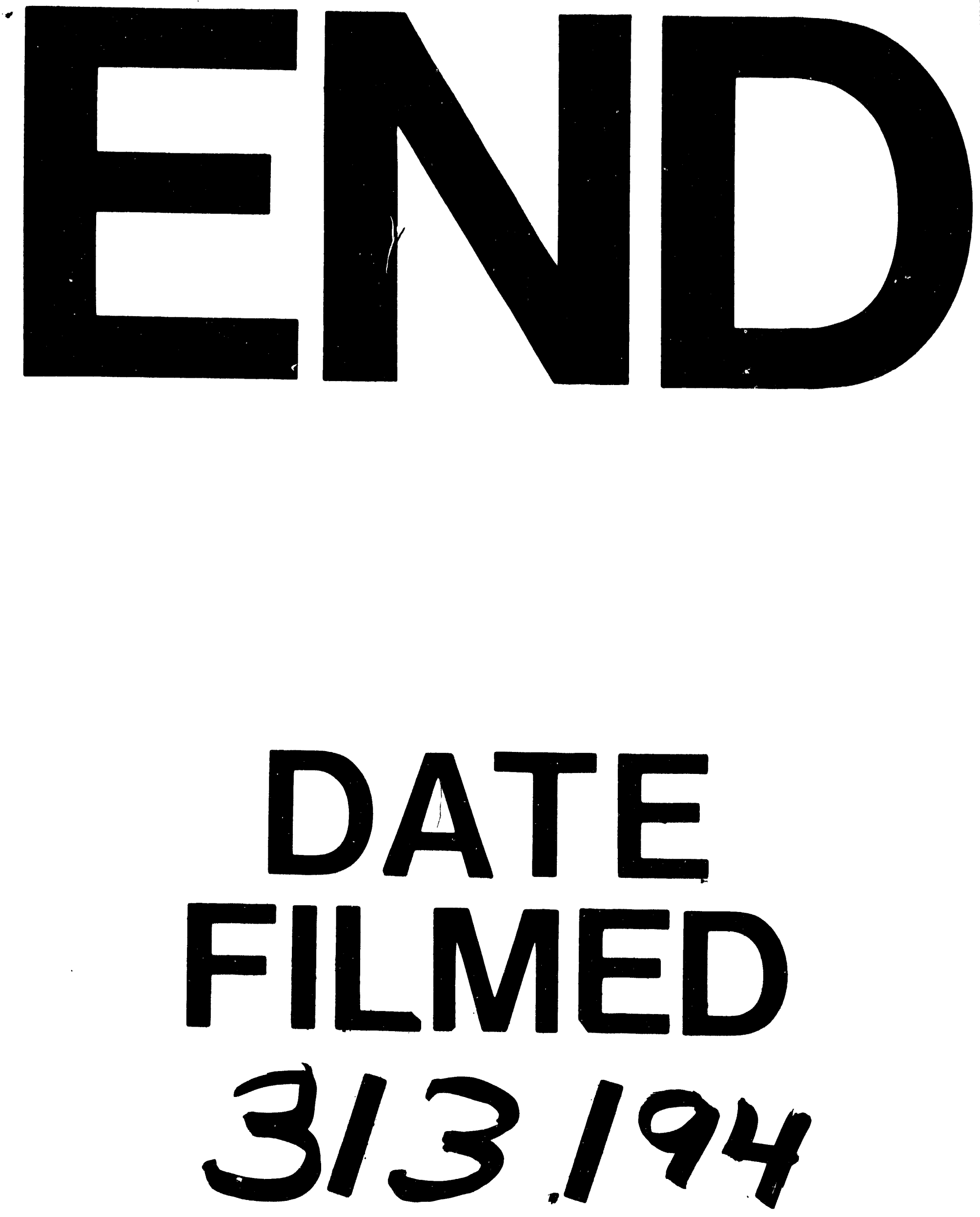
